\title{
SEVEN-COLOR PHOTOMETRY AND CLASSIFICATION OF STARS IN THE VICINITY OF THE EMISSION NEBULA Sh2-205
}

\author{
V. Čepas, J. Zdanavičius, K. Zdanavičius, V. Straižys and V. Laugalys \\ Institute of Theoretical Physics and Astronomy, Vilnius University, \\ Goštauto 12, Vilnius, LT-01108, Lithuania
}

Received: 2013 July 1; accepted 2013 July 22

\begin{abstract}
We present the results of CCD photometry in the seven-color Vilnius system for 922 stars down to $V \sim 17 \mathrm{mag}$ in a 1.5 square degree field at the northern edge of the H II region Sh2-205, at the Perseus and Camelopardalis border. Using the intrinsic color indices and photometric reddening-free $Q$ parameters, two-dimensional spectral types for most stars are determined.
\end{abstract}

Key words: stars: fundamental parameters, classification - H II regions: individual (Sh2-205) - Vilnius photometric system

\section{INTRODUCTION}

Here we continue publishing of the results of CCD photometry and photometric classification of stars in the Vilnius seven-color system in the region of a dust ring of a diameter of $8^{\circ}$, centered on the open cluster NGC 1528 and located at the edge of the Perseus and Camelopardalis constellations (Straižys \& Laugalys 2007, 2008). The first catalog was published by Zdanavičius et al. (2010, Paper I) in the area centered on the open cluster King 7 and covering partly the southern end of the emission nebula Sh2-205. The center of the second area, investigated in the present paper, is at $\alpha(2000)=03^{\mathrm{h}} 57.0^{\mathrm{m}}, \delta(2000)=+53^{\circ} 40^{\prime} ; \ell=148.34^{\circ}$, $b=+0.21^{\circ}$. It covers partly the northern end of the Sh2-205 nebula. This nebula is located at a distance of about $1.0 \mathrm{kpc}$ and belongs to the Local arm. The dust map from Schlegel et al. (1998) shows a very uneven pattern of dust distribution in the area, the densest cloud coincides with the nebula in its western (right-hand) side. According to the atlas of Dobashi et al. (2005), there is one of the clumps of a large dust cloud TGU 942.

As we have noted in Paper I, this area is a part of the $3^{\circ} \times 4^{\circ}$ size region investigated photoelectrically in the Vilnius system by Zdanavičius et al. (2001). Photoelectric photometry for more stars in the area was published by Zdanavičius \& Zdanavičius (2002). The stars from both these papers were used as standards in the present CCD work. The aim of this project is the investigation of the extinction distribution in the vicinity of the nebula Sh2-205, the determination of distances to the dust clouds, and the search of young stellar objects in the area. The Vilnius photometric system and its possibilities are described in detail by Straižys (1992). 


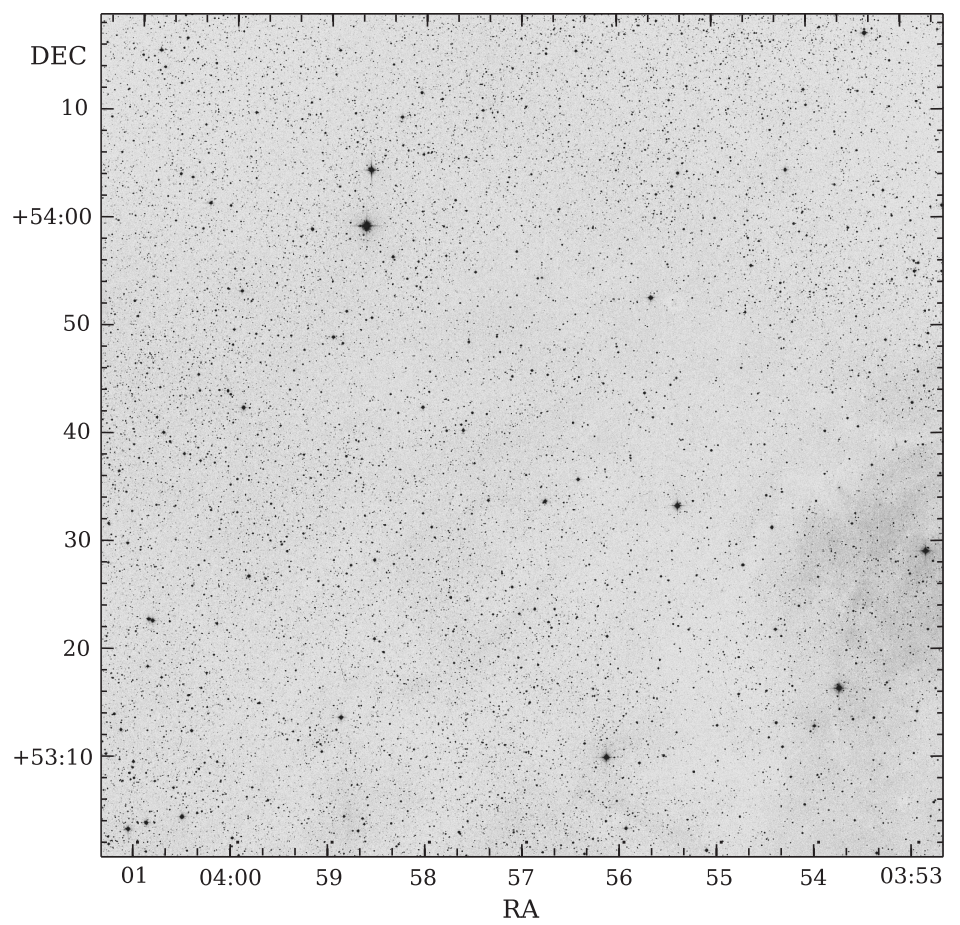

Fig. 1. Map of the observed area in the red filter (DSS1 Red from SkyView).

Table 1. Log of observations. The columns give the filter name, the mean wavelengths of the standard system, the range of exposure lengths and the total number of frames in each filter.

\begin{tabular}{cclc}
\hline Filter & $\lambda_{0}(\mathrm{~nm})$ & Exposure lengths & Number of frames \\
\hline$U$ & 345 & From 1800 s to $60 \mathrm{~s}$ & 12 \\
$P$ & 374 & From 1500 s to 60 s & 8 \\
$X$ & 405 & From 1200 s to 10 s & 9 \\
$Y$ & 466 & From 420 s to 5 s & 9 \\
$Z$ & 516 & From 240 s to 5 s & 8 \\
$V$ & 544 & From 900 s to 5 s & 8 \\
$S$ & 656 & From 240 s to 5 s & 16 \\
\hline
\end{tabular}

\section{OBSERVATIONS AND PROCESSING}

Seven-color CCD photometry in the area shown in Figure 1 was obtained in October of 2010 with the Maksutov-type 35/51 cm telescope of the Moletai Observatory in Lithuania, equipped with a VersArray 1300B liquid nitrogen cooled camera. It has a backside-illuminated $1340 \times 1300$ pixel chip with pixel sizes $20 \times 20 \mu \mathrm{m}$ and with Unichrom UV-enhancement coating. More information about the camera, including its sensitivity function, is given by Zdanavičius \& Zdanavičius (2003). The image scale is $3.38^{\prime \prime}$ per pixel, which results in a field size of $1.26^{\circ} \times 1.22^{\circ}$.

The round Vilnius filters $U P X Y Z V S$ with the $50 \mathrm{~mm}$ diameters were used. The 
Table 2. Internal accuracy of the photometric catalog. The table gives the mean r.m.s. errors in nine magnitude intervals.

\begin{tabular}{rrccccccc}
\hline$V$ interval & \multicolumn{1}{c}{$n$} & $\sigma_{V}$ & $\sigma_{U-V}$ & $\sigma_{P-V}$ & $\sigma_{X-V}$ & $\sigma_{Y-V}$ & $\sigma_{Z-V}$ & $\sigma_{V-S}$ \\
\hline $8.00-9.00$ & 3 & 0.009 & 0.019 & 0.013 & 0.018 & 0.016 & 0.016 & 0.019 \\
$9.00-10.00$ & 4 & 0.009 & 0.015 & 0.012 & 0.027 & 0.014 & 0.015 & 0.027 \\
$10.00-11.00$ & 12 & 0.010 & 0.016 & 0.015 & 0.020 & 0.015 & 0.015 & 0.023 \\
$11.00-12.00$ & 35 & 0.008 & 0.023 & 0.015 & 0.018 & 0.016 & 0.015 & 0.026 \\
$12.00-13.00$ & 97 & 0.009 & 0.021 & 0.016 & 0.019 & 0.015 & 0.015 & 0.022 \\
$13.00-14.00$ & 176 & 0.010 & 0.035 & 0.023 & 0.021 & 0.018 & 0.018 & 0.024 \\
$14.00-15.00$ & 288 & 0.011 & 0.079 & 0.028 & 0.023 & 0.022 & 0.024 & 0.027 \\
$15.00-16.00$ & 158 & 0.014 & 0.090 & 0.035 & 0.032 & 0.023 & 0.026 & 0.029 \\
$16.00-17.00$ & 149 & 0.020 & & & 0.053 & 0.034 & 0.038 & 0.052 \\
\hline
\end{tabular}

mean wavelengths of the response functions are given in Table 1. Other details were described in Paper 1. In total, 70 frames of the area with different exposure lengths were obtained (Table 1). The reduction method of the frames was the same as in Paper I.

Zero-points of the transformation equations of instrumental magnitudes and color indices to the standard Vilnius system, were based on 13 stars in the field observed photoelectrically by Zdanavičius et al. (2001) and Zdanavičius \& Zdanavičius (2002). As the first stage, the linear transformation equations of magnitudes and color indices to the standard system, determined from observations of the M 67 cluster, were applied. The final adjustment of color equations and zero-points has been done by optimizing the accuracy of photometric classification of a selected set of standard stars in the investigated area.

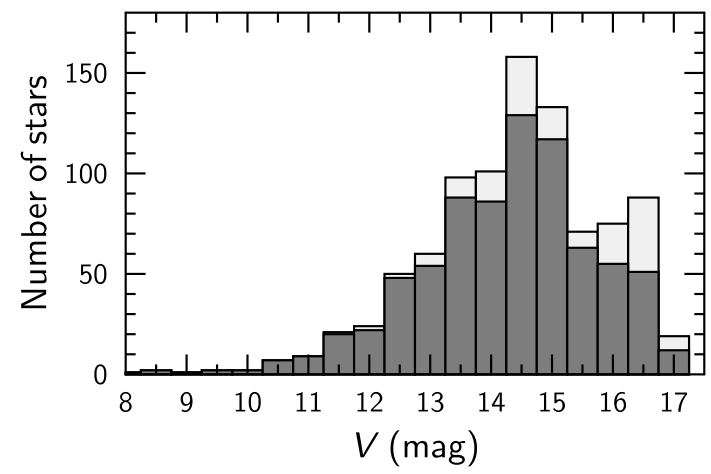

Fig. 2. Distribution of the measured stars in apparent magnitudes. The shadowed parts of the colons correspond to stars with two-dimensional classification.

\section{THE CATALOG}

Magnitudes and colors were determined for 922 stars down to $V=17$ mag. The internal accuracy of the catalog is illustrated in Table 2, where the mean errors in $V$ and color indices in nine magnitude intervals are given. The table shows that the stars brighter than $V=13$ have r.m.s. errors of all indices lower than 0.02 
mag. Down to $V=15$ the errors of $P-V, X-V, Y-V, Z-V$ and $V-S$ do not exceed 0.026 , but the accuracy of $U-V$ becomes twice lower. The $U$ and $P$ magnitudes for the faintest stars with $V$ between magnitudes 16.5 and 17 are not given.

Spectral and luminosity classes are given for about $85 \%$ of stars (Figure 2). The two classification methods, COMPAR and QC0KLAS, were described in Straižys et al. (2013) and Milašius et al. (2013). In calculating of interstellar reddening-free $Q$-parameters the normal interstellar reddening law was applied.

The results of photometry and classification are given in Table 3 . The columns list the following information: star number, equatorial coordinates J2000.0 from PPMXL (Roeser et al. 2010), magnitude $V$, color indices $U-V, P-V, X-V, Y-V$, $Z-V$ and $V-S$, photometric spectral type in the MK system. The color indices with $\sigma=0.05-0.10 \mathrm{mag}$ are marked by colons. In the column of spectral types, the optical binaries with separation $<7^{\prime \prime}$ and the stars with asymmetrical images, i.e., unresolved binaries with separation $<1-2^{\prime \prime}$, are marked by double asterisks. Spectral classes are designated in the lower-case letters to indicate that they are determined from multicolor photometric data.

At the end of Table 3 we give some additional information on identification, binarity, variability, spectral types, etc., taken from the SIMBAD database and other sources. The running numbers of stars having notes at the end of the catalog are marked by asterisks.

ACKNOWLEDGMENTS. The use of the Simbad and SkyView databases is acknowledged. The project is partly supported by the Research Council of Lithuania, grant No. MIP-061/2013.

\section{REFERENCES}

Bouigue M. R. 1959, Annales Obs. Toulouse, 27, 47 = POHP, 4, 52

Boulon J., Duflot M., Fehrenbach C. 1958, J. Obs., 42, 1

Dobashi K., Uehara H., Kandori R. et al. 2005, PASJ, 57, S1

Hartkopf W. I., Mason B. D., Rafferty T. J. 2008, AJ, 135, 1334

Hiltner W. A. 1956, ApJS, 2, 389

Hoffman D. J., Harrison T. E., McNamara B. J. 2009, AJ, 138, 466

Milašius K., Boyle R. P., Vrba F. J. et al. 2013, Baltic Astronomy, 22, 181 (this issue)

Rydstrom B. A. 1978, A\&AS, 32, 25

Roeser S., Demleitner M., Schilbach E. 2010, AJ, 139, 2440

Schlegel D. J., Finkbeiner D. P., Davis M. 1998, ApJ, 500, 525

Straižys V. 1992, Multicolor Stellar Photometry, Pachart Publishing House, Tucson, Arizona; available in pdf format from http://www.itpa.lt/MulticolorStellarPhotometry/

Straižys V., Boyle R. P., Janusz R. et al. 2013, A\&A, 554, A3

Straižys V., Laugalys V. 2007, Baltic Astronomy, 16, 167

Straižys V., Laugalys V. 2008, in Handbook of Star Forming Regions, vol. 1, ed. B. Reipurth, ASP, p. 294

Wozniak P. R., Vestrand W. T., Akerlof C. W. et al. 2004, AJ, 127, 2436 
Zdanavičius J., Čepas V., Zdanavičius K., Straižys V. 2010, Baltic Astronomy, 19, 197 (Paper I)

Zdanavičius J., Černis K. Zdanavičius K., Straižys V. 2001, Baltic Astronomy, 10, 349

Zdanavičius J., Zdanavičius K. 2002, Baltic Astronomy, 11, 75

Zdanavičius J., Zdanavičius K. 2003, Baltic Astronomy, 12, 642

Table 3. Results of photometry and classification of stars in the area. The stars with two asterisks in the last column were not classified in two dimensions since their images are asymmetrical, i.e., these stars are double or multiple. The components of some of them are seen separately but they are closer than $7^{\prime \prime}$.

\begin{tabular}{|c|c|c|c|c|c|c|c|c|c|c|}
\hline No. & $\begin{array}{l}\text { RA }(\mathrm{J} 2000) \\
\mathrm{h} \text { m s }\end{array}$ & $\begin{array}{c}\mathrm{DEC}(\mathrm{J} 2000) \\
\circ, \prime \prime\end{array}$ & $\begin{array}{c}V \\
\text { mag }\end{array}$ & $\begin{array}{l}U-V \\
\text { mag }\end{array}$ & $\begin{array}{l}P-V \\
\text { mag }\end{array}$ & $\begin{array}{l}X-V \\
\text { mag }\end{array}$ & $\begin{array}{l}Y-V \\
\text { mag }\end{array}$ & $\begin{array}{l}Z-V \\
\text { mag }\end{array}$ & $\begin{array}{l}V-S \\
\mathrm{mag}\end{array}$ & $\begin{array}{l}\text { Photom. } \\
\text { sp. type }\end{array}$ \\
\hline 1 & $3: 52: 47.33$ & $+53: 51: 15.0$ & 13.03 & 4.12 & 3.48 & 2.44 & 0.96 & 0.52 & 0.92 & $\mathrm{k} 3.7 \mathrm{~V}$ \\
\hline 2 & $3: 52: 48.50$ & $+53: 44: 18.6$ & 14.56 & & & 3.24 & 1.46 & 0.59 & 1.28 & k0.5 III \\
\hline $3^{*}$ & $3: 52: 48.98$ & $+53: 29: 01.2$ & 8.38 & 1.43 & 1.23 & 0.95 & 0.49 & 0.16 & & $\mathrm{~b}$ \\
\hline 4 & $3: 52: 50.37$ & $+53: 43: 04.2$ & 14.57 & 3.57 & 2.79 & 2.01 & 0.92 & 0.36 & 0.84 & g1.5 III \\
\hline 5 & $3: 52: 52.16$ & $+53: 45: 39.1$ & 13.60 & & 4.39 & 3.12 & 1.37 & 0.53 & 1.23 & k0 III \\
\hline 6 & $3: 52: 53.83$ & $+53: 55: 54.1$ & 13.25 & & 4.20 & 2.97 & 1.29 & 0.51 & 1.16 & k0 III \\
\hline 7 & $3: 52: 55.59$ & $+53: 59: 38.2$ & 13.24 & & 4.87 & 3.49 & 1.51 & 0.60 & 1.34 & k1.5 III \\
\hline 8 & $3: 52: 56.06$ & $+53: 42: 12.5$ & 16.20 & & & 3.09 & 1.38 & 0.54 & 1.31 & k0 IV \\
\hline 9 & $3: 52: 58.39$ & $+53: 54: 21.3$ & 14.27 & 3.21 & 2.38 & 1.59 & 0.75 & 0.28 & 0.66 & f1 ** \\
\hline 10 & $3: 52: 59.05$ & $+53: 22: 09.6$ & 16.47 & & & 2.79 & 1.17 & 0.52 & 1.11 & $\mathrm{k} * *$ \\
\hline 11 & $3: 52: 59.33$ & $+53: 19: 36.3$ & 16.32 & & & 2.80 & 1.30 & 0.56 & 1.22 & $\mathrm{k} 1 \mathrm{~V}$ \\
\hline 12 & $3: 53: 00.17$ & $+53: 44: 52.9$ & 15.99 & & & 2.32 & 0.98 & 0.49 & 0.86 & $\mathrm{k}$ \\
\hline 13 & $3: 53: 03.16$ & $+53: 48: 37.3$ & 16.25 & & & 2.95 & 1.25 & 0.64 & 1.00 & k3 V \\
\hline 14 & $3: 53: 05.70$ & $+53: 44: 30.8$ & 16.88 & & & 2.40 & 0.99 & 0.41 & 0.96 & k0 IV \\
\hline 15 & $3: 53: 07.26$ & $+53: 23: 24.4$ & 15.97 & & & 3.36 & 1.51 & 0.60 & 1.32 & k0.5 III \\
\hline 16 & $3: 53: 07.27$ & $+54: 00: 05.8$ & 14.23 & & 4.36: & 3.17 & 1.39 & 0.54 & 1.24 & k0 III \\
\hline 17 & $3: 53: 07.72$ & $+53: 54: 13.3$ & 13.72 & 3.54 & 2.74 & 2.00 & 0.91 & 0.34 & 0.83 & g1.5 III \\
\hline 18 & $3: 53: 08.41$ & $+53: 38: 24.6$ & 13.62 & 3.24 & 2.45 & 1.75 & 0.83 & 0.31 & 0.73 & f6 IV \\
\hline 19 & $3: 53: 10.63$ & $+53: 28: 18.9$ & 15.99 & & & 2.78 & 1.18 & 0.48 & 1.01 & k0.7 IV \\
\hline 20 & $3: 53: 12.19$ & $+53: 47: 10.3$ & 16.68 & & & 2.62 & 1.08 & 0.50 & 0.97 & k1 IV \\
\hline 21 & $3: 53: 12.25$ & $+53: 48: 37.0$ & 12.29 & 4.86 & 4.09 & 2.85 & 1.19 & 0.48 & 1.04 & k0.7 III \\
\hline 22 & $3: 53: 12.71$ & $+54: 02: 27.4$ & 12.04 & 3.38 & 2.66 & 1.89 & 0.88 & 0.32 & 0.79 & f8 IV \\
\hline 23 & 3:53:13.92 & $+53: 37: 37.7$ & 16.74 & & & 2.44 & 1.03 & 0.45 & 0.90 & k0 IV \\
\hline 24 & $3: 53: 14.24$ & $+53: 16: 53.2$ & 12.93 & & 4.46 & 3.16 & 1.35 & 0.54 & 1.21 & k1 III \\
\hline 25 & $3: 53: 14.39$ & $+53: 56: 12.2$ & 15.54 & & & 3.73 & 1.51 & 0.66 & 1.30 & k3.7 III \\
\hline 26 & $3: 53: 14.43$ & $+53: 43: 06.7$ & 12.49 & 2.93 & 2.21 & 1.47 & 0.69 & 0.26 & 0.59 & f2 IV \\
\hline 27 & $3: 53: 14.53$ & $+53: 08: 08.4$ & 15.23 & & & 2.75 & 1.01 & 0.63 & 1.02 & $\mathrm{k} 5.5 \mathrm{~V}$ \\
\hline 28 & $3: 53: 14.98$ & $+53: 36: 08.1$ & 14.50 & 3.47 & 2.63 & 1.91 & 0.92 & 0.34 & 0.79 & f7 IV \\
\hline 29 & $3: 53: 15.27$ & $+53: 16: 40.2$ & 15.01 & 3.80 & 2.99 & 2.17 & 0.99 & $0.40:$ & 0.89 & g0 IV \\
\hline 30 & $3: 53: 15.75$ & $+53: 18: 36.2$ & 14.72 & 3.82 & 3.03 & 2.17 & 0.95 & 0.39 & 0.92 & g4 IV \\
\hline 31 & $3: 53: 15.78$ & $+53: 52: 39.5$ & 15.29 & & & 2.83 & 1.09 & 0.58 & 1.04 & $\mathrm{k} 3.5 \mathrm{~V}$ \\
\hline 32 & $3: 53: 16.22$ & $+53: 27: 10.7$ & 15.26 & & & 3.52 & 1.55 & 0.63 & 1.40 & k1 III \\
\hline 33 & $3: 53: 17.42$ & $+53: 03: 53.6$ & 16.20 & & & 2.57 & 1.16 & 0.52 & & $\mathrm{k} 1.2 \mathrm{~V}$ \\
\hline 34 & $3: 53: 19.88$ & $+53: 28: 18.8$ & 16.33 & & & $3.24:$ & 1.34 & 0.52 & 1.29 & m3 III \\
\hline 35 & $3: 53: 21.76$ & $+53: 37: 03.2$ & 12.44 & 5.07 & 4.25 & 2.96 & 1.23 & 0.50 & 1.11 & k1.2 III \\
\hline 36 & $3: 53: 22.27$ & $+53: 57: 42.3$ & 15.61 & & & 2.65 & 1.10 & 0.53 & 0.98 & $\mathrm{k} 2.5 \mathrm{~V}$ \\
\hline 37 & $3: 53: 22.49$ & $+53: 45: 37.0$ & 13.36 & 3.28 & 2.42 & 1.53 & 0.71 & 0.26 & 0.60 & a9 IV \\
\hline 38 & $3: 53: 22.53$ & $+53: 13: 38.6$ & 14.05 & & & 4.14 & 1.79 & 0.72 & 1.66 & m1 III \\
\hline 39 & $3: 53: 22.61$ & $+53: 08: 48.0$ & 16.77 & & & 2.60 & 1.06 & 0.48 & 0.97 & k0.7 IV \\
\hline 40 & $3: 53: 22.88$ & $+53: 30: 28.9$ & 14.92 & $3.48:$ & 2.62 & 1.98 & 0.93 & 0.36 & 0.85 & f9 V \\
\hline 41 & $3: 53: 24.39$ & $+53: 48: 13.2$ & 15.32 & 3.43 & 2.70 & 2.02 & 0.90 & 0.32 & 0.79 & g1 IV \\
\hline 42 & $3: 53: 24.71$ & $+53: 16: 14.7$ & 14.30 & 3.42 & 2.61 & 1.92 & 0.89 & 0.34 & 0.79 & f6 IV \\
\hline 43 & $3: 53: 25.00$ & $+53: 47: 00.9$ & 15.09 & 3.52: & 2.79 & 2.02 & 0.90 & 0.38 & 0.80 & g1 IV \\
\hline 44 & $3: 53: 26.66$ & $+53: 52: 20.1$ & 15.21 & & & 3.49 & 1.52 & 0.61 & 1.34 & k1.5 III \\
\hline 45 & $3: 53: 27.85$ & $+53: 36: 07.1$ & 16.53 & & & 2.67: & 1.11 & 0.63 & 1.00 & $\mathrm{k}$ \\
\hline 46 & $3: 53: 28.08$ & $+53: 15: 28.2$ & 16.20 & & & 2.37 & 1.01 & 0.46 & 0.91 & $\mathrm{k} 1.2 \mathrm{~V}$ \\
\hline 47 & $3: 53: 28.29$ & $+53: 35: 41.6$ & 16.69 & & & 2.58 & 1.08 & 0.48 & 1.02 & $\mathrm{k} 1.2 \mathrm{~V}$ \\
\hline 48 & $3: 53: 28.86$ & $+54: 06: 10.5$ & 13.76 & 3.45 & 2.47 & 1.51 & 0.76 & 0.28 & 0.63 & $\mathrm{a} 0.5 \mathrm{IV}$ \\
\hline
\end{tabular}


Table 3. Continued

\begin{tabular}{|c|c|c|c|c|c|c|c|c|c|c|}
\hline No. & $\begin{array}{c}\text { RA (J2000) } \\
\text { h m s }\end{array}$ & $\begin{array}{c}\mathrm{DEC}(\mathrm{J} 2000) \\
\circ, \prime \prime\end{array}$ & $\begin{array}{c}V \\
\text { mag }\end{array}$ & $\begin{array}{l}U-V \\
\text { mag }\end{array}$ & $\begin{array}{l}P-V \\
\text { mag }\end{array}$ & $\begin{array}{l}X-V \\
\text { mag }\end{array}$ & $\begin{array}{l}Y-V \\
\text { mag }\end{array}$ & $\begin{array}{l}Z-V \\
\text { mag }\end{array}$ & $\begin{array}{l}V-S \\
\text { mag }\end{array}$ & $\begin{array}{l}\text { Photom. } \\
\text { sp. type }\end{array}$ \\
\hline 49 & $3: 53: 29.24$ & $+53: 58: 38.4$ & 14.59 & 3.53 & 2.64 & 1.88 & 0.91 & 0.35 & 0.78 & f4 IV \\
\hline 50 & $3: 53: 29.53$ & $+53: 45: 19.9$ & 14.61 & 3.24 & 2.44 & 1.76 & 0.83 & 0.31 & 0.71 & f6 IV \\
\hline 51 & $3: 53: 30.24$ & $+53: 40: 37.4$ & 12.80 & 3.18 & 2.62 & 1.85 & 0.79 & 0.32 & 0.74 & g $5 \mathrm{~V}$ \\
\hline 52 & $3: 53: 30.41$ & $+53: 50: 49.2$ & 13.43 & 2.88 & 2.13 & 1.50 & 0.79 & 0.28 & 0.67 & b6 III: \\
\hline 53 & $3: 53: 32.52$ & $+53: 32: 36.6$ & 13.56 & 3.39 & 2.56 & 1.83 & 0.87 & 0.33 & 0.77 & f7 III \\
\hline 54 & $3: 53: 33.30$ & $+53: 45: 49.8$ & 15.19 & & & 2.43 & 1.01 & 0.46 & 0.95 & $\mathrm{k} 1.5 \mathrm{~V}$ \\
\hline 55 & $3: 53: 33.79$ & $+54: 11: 49.9$ & 14.06 & 3.18 & 2.42 & 1.75 & 0.83 & 0.33 & 0.75 & f $4 \mathrm{~V}$ \\
\hline 56 & $3: 53: 33.81$ & $+53: 29: 30.6$ & 14.43 & 4.44 & 3.65 & 2.65 & 1.15 & 0.43 & 1.08 & g7 $* *$ \\
\hline 57 & $3: 53: 34.41$ & $+53: 23: 33.5$ & 13.97 & 3.98 & 2.99 & 2.18 & 1.04 & 0.39 & 0.91 & f6 III \\
\hline 58 & $3: 53: 34.57$ & $+53: 44: 08.7$ & 14.80 & 3.74 & 2.92 & 2.14 & 0.95 & 0.37 & 0.86 & f7 III \\
\hline 59 & $3: 53: 34.94$ & $+53: 58: 57.9$ & 12.50 & 3.43 & 3.03 & 2.04 & 0.73 & 0.42 & 0.75 & k3 V: \\
\hline 60 & $3: 53: 35.11$ & $+53: 04: 10.6$ & 12.70 & 3.29 & 2.52 & 1.75 & 0.82 & 0.29 & 0.76 & f4 IV \\
\hline 61 & $3: 53: 35.81$ & $+53: 37: 37.9$ & 14.90 & & 3.64: & 2.63 & 0.99 & 0.55 & 0.96 & $\mathrm{k} 3.5 \mathrm{~V}:$ \\
\hline 62 & $3: 53: 36.79$ & $+53: 41: 23.8$ & 13.33 & 3.34 & 2.64 & 1.90 & 0.86 & 0.32 & 0.77 & f9 IV \\
\hline 63 & $3: 53: 36.83$ & $+53: 07: 34.0$ & 15.13 & 3.50 & 2.73 & 2.04 & 0.94 & 0.37 & 0.82 & f7 $\mathrm{V}$ \\
\hline 64 & $3: 53: 38.32$ & $+54: 06: 25.9$ & 14.72 & 3.79 & 3.07 & 2.17 & 0.98 & 0.40 & 0.88 & g5 III \\
\hline 65 & $3: 53: 41.48$ & $+53: 21: 48.9$ & 14.88 & 3.54: & 2.74 & 2.01 & 0.93 & 0.35 & 0.81 & $\mathrm{f} * *$ \\
\hline 66 & $3: 53: 42.03$ & $+53: 39: 26.0$ & 14.75 & 3.48 & 2.68 & 1.97 & 0.91 & $0.35:$ & 0.78 & f7 III \\
\hline 67 & $3: 53: 42.22$ & $+54: 14: 41.8$ & 16.12 & & & 2.98 & 1.28: & 0.71 & 0.99 & $\mathrm{k} * *$ \\
\hline 68 & $3: 53: 42.40$ & $+54: 07: 39.7$ & 16.28 & & & 2.65 & 1.07 & 0.53 & 1.02 & $\mathrm{k} 2.7 \mathrm{~V}$ \\
\hline 69 & $3: 53: 42.80$ & $+53: 06: 51.5$ & 15.02 & & & 2.90 & 1.06 & 0.68 & 1.08 & k1 IV \\
\hline 70 & $3: 53: 43.11$ & $+53: 23: 08.4$ & 14.64 & & & 2.97 & 1.13 & 0.68 & 1.14 & $\mathrm{k} 5.5 \mathrm{~V}$ \\
\hline 71 & $3: 53: 43.17$ & $+54: 03: 02.4$ & 12.60 & 3.58 & 2.67 & 1.66 & 0.79 & 0.28 & 0.66 & a8 III \\
\hline $72^{*}$ & $3: 53: 43.63$ & $+53: 16: 23.0$ & 8.17 & 2.27 & 2.00 & 1.32 & 0.57 & 0.26 & 0.52 & f-g md: \\
\hline 73 & $3: 53: 45.61$ & $+53: 40: 18.7$ & 16.77 & & & 2.46 & 1.04 & 0.48 & 0.90 & $\mathrm{k} 1.2 \mathrm{~V}$ \\
\hline 74 & $3: 53: 45.92$ & $+53: 43: 48.6$ & 13.51 & 3.51 & 2.54 & 1.66 & 0.78 & 0.28 & 0.69 & fO III \\
\hline 75 & $3: 53: 46.26$ & $+53: 46: 05.3$ & 15.62 & & & 3.04 & 1.16 & 0.69 & 1.24 & $\mathrm{k} 8 \mathrm{~V}$ \\
\hline 76 & $3: 53: 47.50$ & $+53: 53: 43.1$ & 14.53 & 3.27 & 2.45 & 1.97 & 1.08 & 0.41 & 0.84 & b6 IV-V: \\
\hline 77 & $3: 53: 47.81$ & $+53: 07: 03.7$ & 16.68 & & & 2.75 & 1.13 & 0.62 & 1.07 & $\mathrm{k}$ \\
\hline 78 & $3: 53: 47.95$ & $+53: 04: 09.4$ & 14.84 & & 3.24 & 2.28 & 0.96 & 0.43 & 0.94 & $\mathrm{k} 0.5 \mathrm{~V}$ \\
\hline 79 & $3: 53: 48.34$ & $+53: 07: 36.9$ & 16.25 & & & 2.69 & 1.06 & 0.58 & 0.99 & $\mathrm{k} 3.5 \mathrm{~V}$ \\
\hline 80 & $3: 53: 48.74$ & $+53: 52: 45.0$ & 14.13 & 3.45 & 2.67 & 1.90 & 0.90 & 0.33 & 0.78 & f5 IV \\
\hline 81 & $3: 53: 48.89$ & $+53: 57: 26.1$ & 14.35 & 3.91 & 3.08 & 2.23 & 0.99 & 0.39 & 0.92 & g3 III \\
\hline 82 & $3: 53: 50.88$ & $+53: 40: 16.1$ & 12.70 & 3.38 & 2.81 & 1.96 & 0.83 & 0.33 & 0.78 & g7 IV \\
\hline 83 & $3: 53: 50.95$ & $+53: 05: 08.2$ & 15.38 & & & 2.38 & 0.98 & 0.46 & 0.91 & $\mathrm{k} 1.5 \mathrm{~V}$ \\
\hline 84 & $3: 53: 51.07$ & $+54: 10: 08.2$ & 16.13 & & & 2.86 & 1.14 & 0.70 & 1.09 & $\mathrm{k} 4 \mathrm{~V}$ \\
\hline 85 & $3: 53: 51.37$ & $+53: 36: 17.1$ & 14.23 & 4.05 & 3.17 & 2.37 & 1.08 & 0.41 & 0.97 & g1.5 III \\
\hline 86 & $3: 53: 52.68$ & $+54: 10: 19.6$ & 14.19 & 3.44 & 2.62 & 1.89 & 0.90 & 0.34 & 0.78 & f5 IV \\
\hline 87 & $3: 53: 54.40$ & $+54: 00: 01.3$ & 16.46 & & & 2.70 & 1.14 & 0.59 & 1.03 & $\mathrm{k} 2.5 \mathrm{~V}$ \\
\hline 88 & $3: 53: 55.49$ & $+53: 53: 47.2$ & 14.69 & 3.44 & 2.64 & 1.96 & 0.90 & 0.33 & 0.80 & f7 IV \\
\hline 89 & $3: 53: 56.05$ & $+53: 26: 44.8$ & 14.41 & & & 3.24 & 1.45 & 0.56 & 1.33 & k0 III \\
\hline 90 & $3: 53: 56.84$ & $+53: 33: 46.9$ & 13.38 & 3.63 & 2.86 & 2.09 & 0.95 & 0.34 & 0.88 & f9.5 IV \\
\hline 91 & $3: 53: 58.22$ & $+53: 44: 30.4$ & 16.49 & & & 2.45 & 1.02 & 0.42 & 1.00 & k0 IV \\
\hline 92 & $3: 53: 58.70$ & $+53: 56: 56.3$ & 14.34 & 3.35 & 2.53 & 1.82 & 0.86 & 0.32 & 0.75 & f5 IV \\
\hline 93 & $3: 53: 59.37$ & $+53: 53: 08.9$ & 14.10 & 3.87 & 2.81 & 1.82 & 0.84 & 0.31 & 0.71 & fO III \\
\hline $94^{*}$ & $3: 53: 59.43$ & $+53: 12: 56.8$ & 11.07 & 2.36 & 1.86 & 1.40 & 0.78 & 0.29 & 0.69 & b2 III-IV: \\
\hline 95 & $3: 54: 00.02$ & $+53: 29: 04.0$ & 16.56 & & & 2.65 & 1.22 & 0.54 & 1.15 & $\mathrm{k} 0.5 \mathrm{~V}$ \\
\hline 96 & $3: 54: 00.79$ & $+54: 10: 26.4$ & 12.12 & 3.54 & 2.96 & 2.10 & 0.92 & 0.36 & 0.84 & g7 V \\
\hline 97 & $3: 54: 01.04$ & $+53: 58: 21.7$ & 13.57 & & 4.43 & 3.17 & 1.36 & 0.54 & 1.21 & $\mathrm{k} 0.5$ III \\
\hline 98 & $3: 54: 01.31$ & $+53: 11: 51.5$ & 16.57 & & & 2.31 & 0.98 & 0.41 & 0.92 & $\mathrm{k} 0.5 \mathrm{~V}$ \\
\hline 99 & $3: 54: 01.80$ & $+53: 12: 27.1$ & 14.79 & & & 3.57 & 1.58 & 0.61 & 1.47 & $\mathrm{k} 2.5$ III \\
\hline 100 & $3: 54: 02.25$ & $+54: 11: 52.5$ & 11.46 & 3.09 & 2.34 & 1.48 & 0.70 & 0.26 & 0.60 & a9 V \\
\hline 101 & $3: 54: 02.72$ & $+53: 55: 19.1$ & 15.78 & & & 3.49 & 1.40 & 0.63 & 1.22 & k3 III \\
\hline 102 & $3: 54: 05.78$ & $+53: 58: 43.8$ & 13.39 & 5.33 & 4.36 & 3.14 & 1.37 & 0.53 & 1.22 & k0 III \\
\hline 103 & $3: 54: 06.08$ & $+53: 05: 38.8$ & 12.80 & 3.33 & 2.79 & 1.89 & 0.80 & 0.32 & 0.75 & g6 IV \\
\hline 104 & $3: 54: 09.02$ & $+53: 30: 50.2$ & 16.21 & & & 3.00 & 1.23 & 0.63 & 1.23 & $\mathrm{k} 3.5 \mathrm{~V}$ \\
\hline 105 & $3: 54: 09.11$ & $+53: 59: 28.1$ & 12.80 & 3.60 & 2.71 & 1.89 & 0.90 & 0.33 & 0.80 & f4 III \\
\hline 106 & $3: 54: 10.62$ & $+53: 50: 23.1$ & 13.96 & 3.57 & 2.53 & 1.68 & 0.81 & 0.29 & 0.65 & a5 III \\
\hline 107 & $3: 54: 10.96$ & $+53: 16: 16.3$ & 14.70 & 3.36 & 2.58 & 1.84 & 0.86 & 0.31 & 0.77 & f5 IV \\
\hline 108 & $3: 54: 12.16$ & $+54: 10: 49.7$ & 15.04 & 3.49 & 2.65 & 1.89 & 0.93 & 0.38 & 0.76 & f3 IV \\
\hline 109 & $3: 54: 13.43$ & $+53: 04: 01.6$ & 16.68 & & & 2.39: & 1.04 & 0.45 & 0.89 & $\mathrm{k} 0 \mathrm{~V}$ \\
\hline 110 & $3: 54: 13.86$ & $+53: 19: 26.1$ & 14.27 & 4.51 & 3.71 & 2.70 & 1.19 & 0.48 & 1.06 & g8 IV \\
\hline
\end{tabular}


Table 3. Continued

\begin{tabular}{|c|c|c|c|c|c|c|c|c|c|c|}
\hline No. & $\begin{array}{c}\text { RA (J2000) } \\
\text { h m s s }\end{array}$ & $\begin{array}{c}\operatorname{DEC}(\mathrm{J} 2000) \\
\circ, \prime \prime\end{array}$ & $\begin{array}{c}V \\
\operatorname{mag}\end{array}$ & $\begin{array}{l}U-V \\
\text { mag }\end{array}$ & $\begin{array}{l}P-V \\
\text { mag }\end{array}$ & $\begin{array}{l}X-V \\
\text { mag }\end{array}$ & $\begin{array}{l}Y-V \\
\text { mag }\end{array}$ & $\begin{array}{l}Z-V \\
\text { mag }\end{array}$ & $\begin{array}{l}V-S \\
\text { mag }\end{array}$ & $\begin{array}{l}\text { Photom. } \\
\text { sp. type }\end{array}$ \\
\hline $111^{*}$ & $3: 54: 14.12$ & $+54: 04: 27.5$ & 10.32 & 1.79 & 1.46 & 0.99 & 0.56 & 0.21 & 0.46 & b3 III: \\
\hline 112 & $3: 54: 14.29$ & $+53: 08: 15.7$ & 12.52 & 3.43 & 2.54 & 1.71 & 0.80 & 0.30 & 0.73 & f5 III \\
\hline 113 & $3: 54: 14.53$ & $+53: 16: 51.4$ & 16.31 & & & 2.83 & 1.09 & 0.59 & 1.10 & $\mathrm{k} 3.7 \mathrm{~V}$ \\
\hline 114 & $3: 54: 14.84$ & $+54: 08: 44.6$ & 13.62 & 3.39 & 2.70 & 1.96 & 0.91 & 0.35 & 0.81 & f9 IV \\
\hline 115 & $3: 54: 16.32$ & $+53: 45: 25.3$ & 14.25 & 3.88 & 3.16 & 2.26 & 1.01 & 0.38 & 0.92 & g3 III \\
\hline 116 & $3: 54: 17.28$ & $+53: 22: 46.4$ & 14.96 & 3.28 & 2.52 & 1.86 & 0.85 & 0.33 & 0.77 & f7 $\mathrm{V}$ \\
\hline 117 & $3: 54: 17.29$ & $+53: 10: 00.3$ & 14.58 & 3.16 & 2.33 & 1.70 & 0.81 & 0.30 & 0.70 & f6 IV \\
\hline 118 & $3: 54: 17.67$ & $+53: 47: 55.2$ & 15.02 & 3.42: & 2.66 & 1.97 & 0.88 & 0.34 & 0.78 & f8 IV \\
\hline 119 & $3: 54: 17.84$ & $+53: 53: 59.7$ & 12.91 & 3.06 & 2.19 & 1.34 & 0.68 & 0.24 & 0.55 & b9 III \\
\hline 120 & $3: 54: 17.88$ & $+53: 53: 08.7$ & 14.45 & 3.94: & 3.21 & 2.33 & 1.03 & 0.45 & 0.86 & $\mathrm{~g} * *$ \\
\hline 121 & $3: 54: 18.08$ & $+53: 34: 39.1$ & 13.45 & 3.40 & 2.64 & 1.95 & 0.88 & 0.33 & 0.81 & $\mathrm{f} 7 \mathrm{~V}$ \\
\hline 122 & $3: 54: 19.23$ & $+53: 11: 02.8$ & 12.28 & 2.43 & 1.86 & 1.39 & 0.76 & 0.28 & 0.68 & b2 III: \\
\hline 123 & $3: 54: 19.27$ & $+53: 28: 53.9$ & 15.62 & & & 2.33 & 0.95 & 0.42 & 0.88 & k0 IV \\
\hline 124 & $3: 54: 19.47$ & $+53: 22: 53.3$ & 15.47 & 3.41 & 2.61 & 1.92 & 0.95 & 0.36 & 0.84 & f $4 \mathrm{~V}$ \\
\hline 125 & $3: 54: 19.53$ & $+54: 03: 40.4$ & 14.91 & 3.67 & 2.72 & 1.94 & 0.93 & 0.35 & 0.81 & f3 III \\
\hline 126 & $3: 54: 21.55$ & $+53: 06: 38.1$ & 16.75 & & & $2.47:$ & 0.99 & 0.48 & 0.94 & $\mathrm{k} 2.5 \mathrm{~V}$ \\
\hline 127 & $3: 54: 22.12$ & $+53: 08: 57.0$ & 14.47 & 3.49 & 2.72 & 1.97 & 0.89 & 0.35 & 0.80 & f9.5 IV \\
\hline 128 & $3: 54: 22.61$ & $+54: 07: 34.1$ & 16.50 & & & 2.50 & 1.08 & 0.49 & 1.01 & $\mathrm{k} 1.5 \mathrm{~V}$ \\
\hline 129 & $3: 54: 23.03$ & $+53: 21: 54.6$ & 11.68 & 4.47 & 3.74 & 2.63 & 1.14 & 0.45 & 1.02 & g9.5 IV \\
\hline 130 & $3: 54: 23.72$ & $+54: 08: 09.2$ & 13.74 & 3.35 & 2.58 & 1.85 & 0.88 & 0.33 & 0.76 & f3 IV \\
\hline 131 & $3: 54: 24.36$ & $+53: 50: 04.2$ & 14.17 & 3.87 & 3.15 & 2.21 & 0.95 & 0.38 & 0.86 & g8 III \\
\hline 132 & $3: 54: 24.60$ & $+53: 31: 21.9$ & 10.79 & 2.73 & 2.12 & 1.41 & 0.65 & 0.23 & 0.61 & f3 V: \\
\hline 133 & $3: 54: 25.43$ & $+53: 34: 15.2$ & 14.53 & 3.48 & 2.62 & 1.93 & 0.88 & 0.33 & 0.77 & f7 III \\
\hline 134 & $3: 54: 26.15$ & $+53: 46: 20.9$ & 14.98 & 3.63: & 2.79 & 2.10 & 1.00 & 0.38 & 0.89 & f8 $\mathrm{V}$ \\
\hline 135 & $3: 54: 26.39$ & $+53: 21: 13.9$ & 14.41 & 3.74 & 3.06 & 2.14 & 0.92 & 0.39 & 0.87 & g9 V \\
\hline 136 & $3: 54: 27.60$ & $+53: 11: 55.5$ & 12.60 & 3.07 & 2.28 & 1.52 & 0.72 & 0.26 & 0.64 & f2 IV: \\
\hline 137 & $3: 54: 27.94$ & $+53: 34: 20.0$ & 12.59 & 3.17 & 2.30 & 1.33 & 0.63 & 0.23 & 0.51 & $\mathrm{a} 2 \mathrm{~V}$ \\
\hline 138 & $3: 54: 27.94$ & $+54: 09: 14.3$ & 14.13 & & 4.44: & 3.11 & 1.31 & 0.53 & 1.13 & $\mathrm{k} 1.2$ III \\
\hline 139 & $3: 54: 28.13$ & $+54: 15: 55.0$ & 13.41 & & 4.73 & 3.30 & 1.38 & 0.58 & 1.18 & k2 III \\
\hline 140 & $3: 54: 28.64$ & $+54: 03: 10.8$ & 13.62 & 3.56 & 2.58 & 1.61 & 0.77 & 0.26 & 0.65 & a7 III \\
\hline 141 & $3: 54: 29.59$ & $+54: 00: 02.4$ & 16.70 & & & 2.54 & 1.11 & 0.48 & 1.07 & $\mathrm{k} 0.7 \mathrm{~V}$ \\
\hline 142 & $3: 54: 29.70$ & $+54: 16: 24.1$ & 14.40 & 3.32 & 2.60 & 1.87 & 0.83 & 0.39 & 0.77 & $f-g$ \\
\hline 143 & $3: 54: 29.82$ & $+54: 04: 51.8$ & 14.65 & & 3.95 & 2.75 & 1.03 & 0.58 & 1.04 & $\mathrm{k} 4 \mathrm{~V}$ : \\
\hline 144 & $3: 54: 30.26$ & $+54: 14: 04.1$ & 15.92 & & & 3.15 & 1.20 & 0.77 & 1.01 & $\mathrm{k} 5.5 \mathrm{~V}$ \\
\hline 145 & $3: 54: 30.40$ & $+53: 28: 23.5$ & 15.47 & & & 2.33 & 0.96 & 0.44 & 0.86 & $\mathrm{k} 1 \mathrm{~V}$ \\
\hline 146 & $3: 54: 30.81$ & $+53: 52: 16.6$ & 15.12 & 3.48 & 2.63 & 1.88 & 0.88 & 0.32 & 0.77 & f4 III \\
\hline 147 & $3: 54: 31.11$ & $+53: 57: 52.2$ & 13.49 & 5.30 & 4.40 & 3.27 & 1.38 & 0.52 & 1.18 & k0.5 III \\
\hline 148 & $3: 54: 31.71$ & $+53: 22: 20.9$ & 15.08 & 3.60 & 2.85 & 2.12 & 0.90 & 0.35 & 0.85 & g2 III \\
\hline 149 & $3: 54: 31.88$ & $+53: 36: 03.7$ & 16.38 & & & 2.59 & 1.23 & 0.46 & 1.03 & $\mathrm{k}-\mathrm{m}$ \\
\hline 150 & $3: 54: 32.52$ & $+53: 43: 46.6$ & 14.39 & 3.88 & 3.12 & 2.25 & 1.02 & 0.38 & 0.87 & $\mathrm{~g} * *$ \\
\hline 151 & $3: 54: 32.86$ & $+53: 33: 00.2$ & 15.86 & & & 2.53 & 1.04 & 0.51 & 0.93 & $\mathrm{k} 2.2 \mathrm{~V}$ \\
\hline 152 & $3: 54: 32.89$ & $+53: 16: 50.8$ & 15.50 & & & 3.16 & 1.16 & 0.77 & 1.18 & $\mathrm{k}-\mathrm{m} * *$ \\
\hline 153 & $3: 54: 33.00$ & $+53: 40: 10.3$ & 16.70 & & & 2.37 & 1.02 & 0.44 & 0.90 & $\mathrm{k} 0 \mathrm{~V}$ \\
\hline 154 & $3: 54: 33.90$ & $+53: 52: 12.3$ & 14.54 & 3.64 & 2.60 & 1.65 & 0.79 & 0.28 & 0.64 & a8 III \\
\hline 155 & $3: 54: 34.70$ & $+53: 21: 09.2$ & 15.78 & & & 2.43 & 1.00 & 0.46 & 0.97 & $\mathrm{k} 1.2 \mathrm{~V}$ \\
\hline 156 & $3: 54: 35.19$ & $+53: 52: 44.1$ & 14.70 & 3.73: & 2.86 & 2.35 & 1.24 & 0.43 & 1.10 & $\mathrm{a} * *$ \\
\hline 157 & $3: 54: 35.53$ & $+53: 16: 22.4$ & 16.53 & & & 2.72 & 1.29 & 0.57 & 1.13 & g6 V \\
\hline 158 & $3: 54: 36.61$ & $+53: 55: 37.6$ & 11.61 & 2.80 & 2.24 & 1.59 & 0.74 & 0.28 & 0.66 & f6 $\mathrm{V}$ \\
\hline 159 & $3: 54: 36.97$ & $+53: 57: 02.0$ & 15.26 & 3.77 & 2.74 & 1.96 & 1.09 & 0.44 & 0.88 & $\mathrm{~b} * *$ \\
\hline 160 & $3: 54: 37.01$ & $+54: 13: 20.7$ & 14.84 & 3.36 & 2.69 & 1.93 & 0.88 & 0.36 & 0.80 & g0 IV \\
\hline 161 & $3: 54: 37.02$ & $+53: 39: 03.5$ & 15.05 & 3.66 & 3.07 & 2.14 & 0.94 & 0.38 & 0.86 & g8 IV \\
\hline 162 & $3: 54: 38.04$ & $+53: 58: 10.7$ & 13.34 & 3.50 & 2.60 & 1.75 & 0.86 & 0.33 & 0.69 & fO III \\
\hline 163 & $3: 54: 38.12$ & $+53: 59: 47.8$ & 13.99 & & & 3.35 & 1.34 & 0.62 & 1.21 & k3 III \\
\hline 164 & $3: 54: 38.44$ & $+53: 18: 20.5$ & 13.88 & 3.08 & 2.30 & 1.54 & 0.72 & 0.27 & 0.62 & f2 IV \\
\hline 165 & $3: 54: 38.63$ & $+54: 07: 15.5$ & 16.64 & & & 2.75 & 1.08 & 0.57 & 1.10 & $\mathrm{k} 3.5 \mathrm{~V}$ \\
\hline 166 & $3: 54: 38.65$ & $+53: 25: 48.1$ & 16.94 & & & 2.35 & 0.96 & 0.39 & 0.99 & k0 IV \\
\hline 167 & $3: 54: 38.74$ & $+54: 02: 11.6$ & 13.53 & 3.86 & 3.28 & 2.29 & 0.91 & 0.43 & 0.88 & $\mathrm{k} 1.5 \mathrm{~V}:$ \\
\hline 168 & $3: 54: 40.03$ & $+53: 51: 55.8$ & 14.70 & 3.98: & 3.35 & 2.33 & 0.99 & 0.43 & 0.89 & $\mathrm{k} 0.5 \mathrm{~V}$ \\
\hline 169 & $3: 54: 40.12$ & $+53: 51: 18.1$ & 12.20 & 3.30 & 2.37 & 1.45 & 0.74 & 0.27 & 0.61 & b9 IV \\
\hline 170 & $3: 54: 40.16$ & $+53: 59: 25.3$ & 14.95 & 3.81 & 2.84 & 2.07 & 0.95 & 0.35 & 0.85 & f7 III \\
\hline 171 & $3: 54: 40.55$ & $+53: 56: 26.4$ & 14.67 & 3.65: & 2.69 & 1.84 & 0.79 & 0.30 & 0.62 & g5 IV \\
\hline 172 & $3: 54: 43.01$ & $+53: 46: 09.3$ & 12.53 & 3.13 & 2.28 & 1.30 & 0.60 & 0.21 & 0.49 & a5 IV \\
\hline
\end{tabular}


Table 3. Continued

\begin{tabular}{|c|c|c|c|c|c|c|c|c|c|c|}
\hline No. & $\begin{array}{c}\text { RA (J2000) } \\
\text { h m s s }\end{array}$ & $\begin{array}{c}\operatorname{DEC}(\mathrm{J} 2000) \\
\circ, \prime \prime\end{array}$ & $\begin{array}{c}V \\
\operatorname{mag}\end{array}$ & $\begin{array}{l}U-V \\
\text { mag }\end{array}$ & $\begin{array}{l}P-V \\
\text { mag }\end{array}$ & $\begin{array}{l}X-V \\
\text { mag }\end{array}$ & $\begin{array}{l}Y-V \\
\text { mag }\end{array}$ & $\begin{array}{l}Z-V \\
\text { mag }\end{array}$ & $\begin{array}{l}V-S \\
\text { mag }\end{array}$ & $\begin{array}{l}\text { Photom. } \\
\text { sp. type }\end{array}$ \\
\hline 173 & $3: 54: 43.07$ & $+54: 03: 23.7$ & 16.59 & & & 2.56 & 1.08 & 0.53 & 1.00 & $\mathrm{k} 2 \mathrm{~V}$ \\
\hline 174 & $3: 54: 43.20$ & $+53: 27: 50.7$ & 11.59 & & 3.35 & 2.23 & 0.78 & 0.49 & 0.85 & k3.7 V: \\
\hline 175 & $3: 54: 43.73$ & $+53: 51: 12.1$ & 16.09 & & & 2.58 & 1.07 & 0.47 & 0.94 & k0.7 IV \\
\hline 176 & $3: 54: 45.90$ & $+53: 19: 22.8$ & 12.97 & 2.72 & 1.92 & 1.12 & 0.57 & 0.21 & 0.45 & b9 \\
\hline 177 & $3: 54: 46.19$ & $+53: 15: 57.4$ & 11.79 & 2.91 & 2.27 & 1.58 & 0.73 & 0.27 & 0.67 & f $4 \mathrm{~V}$ \\
\hline 178 & $3: 54: 46.21$ & $+53: 04: 37.1$ & 13.49 & 3.20 & 2.59 & 1.84 & 0.82 & 0.32 & 0.78 & g1 V \\
\hline 179 & $3: 54: 47.18$ & $+53: 34: 48.8$ & 16.65 & & & 2.67 & 1.14 & 0.49 & 1.03 & $\mathrm{k} 0.5 \mathrm{~V}$ \\
\hline 180 & $3: 54: 48.45$ & $+54: 05: 19.4$ & 14.30 & 3.49 & 2.75 & 2.00 & 0.91 & 0.34 & 0.82 & f9 IV \\
\hline 181 & $3: 54: 48.68$ & $+54: 05: 39.8$ & 13.86 & 3.29 & 2.60 & 1.90 & 0.88 & 0.34 & 0.77 & f9 IV \\
\hline 182 & $3: 54: 49.05$ & $+54: 12: 57.8$ & 13.10 & 3.27 & 2.40 & 1.44 & 0.68 & 0.26 & 0.56 & a6 IV \\
\hline 183 & $3: 54: 49.97$ & $+54: 10: 09.2$ & 14.34 & 3.80 & 2.73 & 1.78 & 0.86 & 0.31 & 0.73 & a8 III \\
\hline 184 & $3: 54: 51.31$ & $+54: 11: 59.6$ & 13.88 & 3.13 & 2.23 & 1.48 & 0.79 & 0.29 & 0.64 & b7 III \\
\hline 185 & $3: 54: 51.87$ & $+53: 51: 26.5$ & 16.38 & & & 2.44 & 1.04 & 0.46 & 0.94 & k0.5 IV \\
\hline 186 & $3: 54: 52.90$ & $+54: 01: 21.4$ & 16.52 & & & 2.17 & 0.99 & 0.30 & 1.06 & g-k \\
\hline 187 & $3: 54: 53.53$ & $+53: 29: 05.4$ & 14.67 & 3.51 & 2.60 & 1.74 & 0.81 & 0.31 & 0.68 & f2 III \\
\hline 188 & $3: 54: 54.08$ & $+54: 06: 31.1$ & 14.81 & 3.70: & 2.93 & 2.16 & 0.97 & 0.38 & 0.86 & g1 IV \\
\hline 189 & $3: 54: 55.04$ & $+54: 16: 19.6$ & 13.86 & 3.49 & 2.48 & 1.54 & 0.74 & 0.27 & 0.62 & a4 III \\
\hline 190 & $3: 54: 55.43$ & $+53: 18: 00.8$ & 16.64 & & & 2.30 & 0.96 & 0.53 & 0.92 & $\mathrm{k} 1 * *$ \\
\hline 191 & $3: 54: 55.80$ & $+53: 36: 14.7$ & 15.96 & & & 2.47 & 1.04 & 0.45 & 1.01 & $\mathrm{k} 1 \mathrm{~V}$ \\
\hline 192 & $3: 54: 56.78$ & $+54: 04: 16.8$ & 13.33 & 3.28 & 2.68 & 1.90 & 0.83 & 0.32 & 0.76 & g5 V \\
\hline 193 & $3: 54: 56.79$ & $+53: 04: 58.8$ & 15.71 & & & 2.33 & 0.95 & 0.48 & 0.87 & $\mathrm{k} 2 \mathrm{~V}$ \\
\hline 194 & $3: 54: 57.71$ & $+53: 46: 55.5$ & 16.18 & & & 2.24 & 0.98 & 0.44 & 0.92 & $\mathrm{k} 0 \mathrm{~V}$ \\
\hline 195 & $3: 54: 58.48$ & $+54: 11: 00.8$ & 15.04 & 3.83 & 3.21 & 2.34 & 1.01 & 0.41 & 0.92 & g8 IV \\
\hline 196 & $3: 54: 58.79$ & $+53: 44: 59.6$ & 16.30 & & & 2.47 & 1.07 & 0.42 & 0.92 & g9 \\
\hline 197 & $3: 54: 58.88$ & $+54: 00: 02.6$ & 12.86 & 3.22 & 2.36 & 1.38 & 0.67 & 0.23 & 0.53 & a3 IV \\
\hline 198 & $3: 54: 58.97$ & $+53: 42: 07.7$ & 16.00 & & & 3.05 & 1.26 & 0.56 & 1.18 & k1 IV \\
\hline 199 & $3: 54: 59.32$ & $+53: 09: 47.8$ & 14.38 & 3.69 & 3.01 & 2.13 & 0.89 & 0.40 & 0.84 & $\mathrm{k} 0 \mathrm{~V}$ \\
\hline 200 & $3: 54: 59.57$ & $+53: 13: 05.6$ & 12.52 & 3.02 & 2.39 & 1.67 & 0.75 & 0.28 & 0.70 & f8 IV \\
\hline 201 & $3: 55: 00.13$ & $+54: 15: 28.5$ & 14.03 & 3.52 & 2.62 & 1.61 & 0.78 & 0.28 & 0.65 & a6 IV \\
\hline 202 & $3: 55: 01.98$ & $+53: 38: 31.7$ & 13.18 & 3.46 & 2.65 & 1.89 & 0.88 & 0.32 & 0.77 & f5 III \\
\hline 203 & $3: 55: 02.06$ & $+53: 13: 03.1$ & 15.95 & & & 2.49 & 0.92 & 0.50 & 0.98 & $\mathrm{k} 3.5 \mathrm{~V}$ \\
\hline 204 & $3: 55: 03.84$ & $+53: 29: 46.8$ & 15.86 & & & 2.38 & 0.95 & 0.42 & 0.94 & k0 IV \\
\hline 205 & $3: 55: 04.47$ & $+54: 07: 10.4$ & 16.41 & & & 2.98: & 1.13 & 0.70: & 1.07 & $\mathrm{k} 6 \mathrm{~V}$ \\
\hline 206 & $3: 55: 04.59$ & $+53: 18: 02.8$ & 13.40 & 3.18 & 2.42 & 1.70 & 0.79 & 0.29 & 0.72 & f4 IV \\
\hline 207 & $3: 55: 05.29$ & $+53: 38: 53.0$ & 14.62 & 3.58 & 2.68 & 1.98 & 0.90 & 0.34 & 0.79 & f9 $\mathrm{V}$ \\
\hline 208 & $3: 55: 06.06$ & $+53: 23: 03.7$ & 15.04 & 3.72 & 2.92 & 2.05 & 0.94 & 0.36 & 0.86 & g5 V \\
\hline 209 & $3: 55: 07.20$ & $+53: 26: 35.1$ & 15.66 & & & 2.59 & 1.01 & 0.52 & 0.97 & $\mathrm{k} 2.7 \mathrm{~V}$ \\
\hline 210 & $3: 55: 07.27$ & $+53: 58: 50.9$ & 13.62 & & 3.88 & 2.71 & 1.14 & 0.48 & 1.02 & k0.5 III \\
\hline 211 & $3: 55: 07.87$ & $+53: 24: 07.8$ & 15.05 & 3.46: & 2.74 & 1.98 & 0.89 & 0.34 & 0.82 & g0 IV \\
\hline 212 & $3: 55: 09.36$ & $+54: 14: 24.7$ & 16.02 & & & 2.36 & 1.03: & 0.47 & 0.93 & $\mathrm{k} 0.7 \mathrm{~V}$ \\
\hline 213 & $3: 55: 10.78$ & $+53: 29: 29.2$ & 14.57 & 3.98: & 3.25 & 2.34 & 1.04 & 0.41 & 0.92 & g5 IV \\
\hline 214 & $3: 55: 11.35$ & $+54: 08: 21.3$ & 13.45 & 3.28 & 2.40 & 1.46 & 0.67 & 0.24 & 0.53 & a8 III \\
\hline 215 & $3: 55: 11.50$ & $+53: 27: 00.3$ & 13.02 & 3.21 & 2.36 & 1.43 & 0.68 & 0.25 & 0.56 & a $* *$ \\
\hline 216 & $3: 55: 13.58$ & $+53: 33: 52.7$ & 13.55 & 3.92 & 2.85 & 1.84 & 0.87 & 0.30 & 0.76 & a9 III \\
\hline 217 & $3: 55: 13.91$ & $+53: 16: 36.1$ & 15.66 & & & 3.12 & 1.19 & 0.70 & 1.23 & $\mathrm{k} 6 \mathrm{~V}$ \\
\hline 218 & $3: 55: 14.15$ & $+53: 08: 47.0$ & 13.58 & 4.92: & 4.13 & 2.85 & 1.15 & 0.50 & 1.05 & k1.7 III \\
\hline 219 & $3: 55: 14.78$ & $+54: 02: 03.2$ & 14.91 & & & 2.87 & 1.25 & 0.51 & 1.09 & k0.5 III \\
\hline 220 & $3: 55: 14.98$ & $+53: 57: 43.5$ & 15.88 & & & 2.85 & 1.16 & 0.67 & 1.23 & k3 V \\
\hline 221 & $3: 55: 15.47$ & $+53: 39: 06.6$ & 16.47 & & & 2.75 & 1.20 & 0.57 & 0.97 & $\mathrm{k} 1.5 \mathrm{~V}$ \\
\hline 222 & $3: 55: 16.21$ & $+54: 12: 15.9$ & 12.91 & 4.62 & 3.85 & 2.72 & 1.15 & 0.48 & 1.01 & k0 III \\
\hline 223 & $3: 55: 16.27$ & $+53: 54: 37.9$ & 16.13 & & & 2.91 & 1.21 & 0.57 & 1.28 & k3 V \\
\hline 224 & $3: 55: 16.34$ & $+54: 04: 22.8$ & 12.66 & 3.40 & 2.53 & 1.61 & 0.74 & 0.28 & 0.62 & a9 IV \\
\hline 225 & $3: 55: 16.64$ & $+53: 21: 59.5$ & 13.01 & 3.34 & 2.80 & 1.93 & 0.80 & 0.34 & 0.78 & $\mathrm{~g} 8.5 \mathrm{~V}$ \\
\hline 226 & $3: 55: 18.18$ & $+53: 59: 00.7$ & 13.63 & 3.33 & 2.72 & 1.98 & 0.85 & 0.35 & 0.81 & g6 IV \\
\hline 227 & $3: 55: 18.21$ & $+53: 04: 22.4$ & 13.55 & 3.19 & 2.31 & 1.49 & 0.71 & 0.26 & 0.63 & fO III \\
\hline 228 & $3: 55: 18.72$ & $+53: 54: 17.3$ & 15.36 & 3.45 & 2.67 & 2.01 & 0.92 & 0.34 & 0.82 & f9.5 V \\
\hline 229 & $3: 55: 19.01$ & $+53: 38: 37.8$ & 15.25 & & & 3.80 & 1.72 & 0.65 & 1.54 & k0.5 III \\
\hline 230 & $3: 55: 21.08$ & $+53: 45: 49.7$ & 15.44 & & & 2.32 & 1.00 & 0.43 & 0.93 & $\mathrm{k} 0.7 \mathrm{~V}$ \\
\hline 231 & $3: 55: 21.17$ & $+53: 23: 09.3$ & 13.79 & 3.29 & 2.42 & 1.56 & 0.74 & 0.27 & 0.63 & fo IV \\
\hline 232 & $3: 55: 22.02$ & $+53: 19: 55.1$ & 14.85 & 3.11 & 2.35 & 1.70 & 0.80 & 0.29 & 0.70 & f7 IV \\
\hline 233 & $3: 55: 22.04$ & $+54: 04: 12.8$ & 11.58 & 3.23 & 2.48 & 1.73 & 0.82 & 0.30 & 0.72 & f5 IV \\
\hline 234 & $3: 55: 22.32$ & $+53: 20: 13.7$ & 14.76 & 3.18 & 2.37 & 1.70 & 0.79 & 0.30 & 0.70 & f4 IV \\
\hline
\end{tabular}


Table 3. Continued

\begin{tabular}{|c|c|c|c|c|c|c|c|c|c|c|}
\hline No. & $\begin{array}{c}\text { RA (J2000) } \\
\text { h m s }\end{array}$ & $\begin{array}{c}\mathrm{DEC}(\mathrm{J} 2000) \\
\circ, \prime \prime\end{array}$ & $\begin{array}{c}V \\
\text { mag }\end{array}$ & $\begin{array}{l}U-V \\
\text { mag }\end{array}$ & $\begin{array}{l}P-V \\
\text { mag }\end{array}$ & $\begin{array}{l}X-V \\
\text { mag }\end{array}$ & $\begin{array}{l}Y-V \\
\text { mag }\end{array}$ & $\begin{array}{l}Z-V \\
\text { mag }\end{array}$ & $\begin{array}{l}V-S \\
\text { mag }\end{array}$ & $\begin{array}{l}\text { Photom. } \\
\text { sp. type }\end{array}$ \\
\hline $235^{*}$ & $3: 55: 23.00$ & $+53: 33: 27.3$ & 8.48 & 2.10 & 1.77 & 1.15 & 0.51 & 0.24 & 0.48 & $\mathrm{f} 7 \mathrm{~V}, \mathrm{md}:$ \\
\hline 236 & $3: 55: 23.20$ & $+54: 08: 17.1$ & 16.28 & & & 2.65 & 1.13 & 0.47 & 1.02 & $\mathrm{k} 0.5 \mathrm{IV}$ \\
\hline 237 & $3: 55: 23.61$ & $+53: 45: 15.0$ & 12.11 & 3.47 & 2.67 & 1.91 & 0.89 & 0.32 & 0.80 & f4 IV \\
\hline 238 & $3: 55: 23.73$ & $+53: 17: 19.1$ & 16.75 & & & 2.52 & 1.18 & 0.59 & 0.98 & g5 \\
\hline 239 & $3: 55: 23.92$ & $+54: 11: 26.5$ & 15.95 & & & 3.22 & 1.22 & 0.73 & 1.25 & $\mathrm{k} 8 \mathrm{~V}$ \\
\hline 240 & $3: 55: 24.61$ & $+53: 12: 27.0$ & 12.95 & 2.90 & 2.19 & 1.43 & 0.68 & 0.26 & 0.59 & f2 IV \\
\hline 241 & $3: 55: 24.91$ & $+54: 12: 16.6$ & 15.76 & & & 2.74 & 1.07 & 0.64 & 0.92 & $\mathrm{k} 4 \mathrm{~V}: * *$ \\
\hline 242 & $3: 55: 25.58$ & $+53: 47: 01.5$ & 15.19 & 3.54 & 2.86 & 2.06 & 0.95 & 0.34 & 0.87 & f9 IV \\
\hline 243 & $3: 55: 25.89$ & $+54: 02: 58.7$ & 12.02 & 3.03 & 2.34 & 1.63 & 0.77 & 0.28 & 0.69 & f3 $\mathrm{V}$ \\
\hline 244 & $3: 55: 25.94$ & $+54: 08: 54.0$ & 14.13 & 3.59 & 2.63 & 1.83 & 0.87 & 0.32 & 0.76 & f3 III \\
\hline 245 & $3: 55: 26.44$ & $+53: 22: 28.6$ & 15.07 & 3.51: & 2.68 & 1.95 & 0.91 & 0.34 & 0.80 & f7 IV \\
\hline 246 & $3: 55: 26.75$ & $+54: 11: 25.9$ & 16.21 & & & 3.11 & 1.19 & 0.73 & 1.17 & $\mathrm{k} 5.5 \mathrm{~V}$ \\
\hline 247 & $3: 55: 26.93$ & $+53: 29: 16.3$ & 14.98 & 3.82 & 2.87 & 2.14 & 0.99 & 0.36 & 0.88 & f8 IV \\
\hline 248 & $3: 55: 26.95$ & $+53: 11: 02.0$ & 16.69 & & & 2.26 & 0.96 & 0.49 & 0.87 & $\mathrm{k} 0 \mathrm{~V}$ \\
\hline 249 & $3: 55: 27.44$ & $+53: 44: 36.6$ & 12.87 & 3.24 & 2.64 & 1.85 & 0.82 & 0.31 & 0.76 & $\mathrm{~g} 4 \mathrm{~V}$ \\
\hline 250 & $3: 55: 27.51$ & $+54: 10: 58.8$ & 12.55 & 3.17 & 2.51 & 1.78 & 0.82 & 0.31 & 0.71 & f8 IV \\
\hline 251 & $3: 55: 28.08$ & $+53: 16: 10.9$ & 15.37 & 3.37: & 2.60 & 1.93 & 0.90 & 0.38 & 0.77 & $\mathrm{f} 7 \mathrm{~V}$ \\
\hline 252 & $3: 55: 28.10$ & $+53: 58: 44.6$ & 16.16 & & & 2.68 & 1.07 & 0.43 & 1.04 & $\mathrm{k}$ \\
\hline 253 & $3: 55: 28.73$ & $+53: 24: 30.1$ & 12.40 & 3.15 & 2.40 & 1.65 & 0.79 & 0.28 & 0.71 & f3 IV \\
\hline 254 & $3: 55: 28.75$ & $+53: 47: 15.9$ & 16.36 & & & 2.67 & 1.05 & 0.58 & 1.04 & $\mathrm{k} 3.7 \mathrm{~V}$ \\
\hline 255 & $3: 55: 28.88$ & $+53: 55: 47.1$ & 14.37 & 4.65 & 3.81 & 2.71 & 1.14 & 0.49 & 1.03 & k0.5 IV \\
\hline 256 & $3: 55: 28.95$ & $+54: 00: 06.6$ & 15.91 & & & 2.92 & 1.12 & 0.66 & 1.08 & k5 V \\
\hline 257 & $3: 55: 29.26$ & $+53: 26: 32.3$ & 13.65 & & 4.79 & 3.31 & 1.32 & 0.58 & 1.20 & k3 III \\
\hline 258 & $3: 55: 30.82$ & $+53: 25: 04.8$ & 15.26 & 3.35 & 2.54 & 1.83 & 0.85 & 0.30 & 0.76 & f8 IV \\
\hline 259 & $3: 55: 31.46$ & $+53: 57: 23.4$ & 14.28 & 3.56 & 2.63 & 1.73 & 0.81 & 0.30 & 0.67 & fO III \\
\hline 260 & $3: 55: 31.70$ & $+53: 05: 14.3$ & 15.28 & & & 2.58 & 0.95 & 0.57 & 0.99 & $\mathrm{k} 4 \mathrm{~V}$ \\
\hline 261 & $3: 55: 32.91$ & $+53: 10: 17.1$ & 12.25 & 3.09 & 2.57 & 1.76 & 0.75 & 0.31 & 0.73 & g5.5 V \\
\hline 262 & $3: 55: 33.02$ & $+54: 15: 22.7$ & 16.38 & & & 2.51 & 1.04 & 0.48 & 0.96 & $\mathrm{k} 1.7 \mathrm{~V}$ \\
\hline 263 & $3: 55: 33.82$ & $+53: 26: 53.1$ & 13.53 & 3.70 & 2.89 & 2.08 & 0.94 & 0.37 & 0.85 & g4 IV-V \\
\hline 264 & $3: 55: 33.95$ & $+53: 14: 19.1$ & 15.91 & & & 2.41 & 1.00 & 0.46 & 0.94 & $\mathrm{k} 1.5 \mathrm{~V}$ \\
\hline 265 & $3: 55: 34.17$ & $+53: 56: 49.3$ & 16.69 & & & 2.54 & 1.12 & 0.49 & 1.04 & $\mathrm{k} 2 \mathrm{~V}$ \\
\hline 266 & $3: 55: 34.18$ & $+53: 59: 01.5$ & 16.56 & & & 2.29 & 0.96 & 0.42 & 0.97 & $\mathrm{k} 1.2 \mathrm{~V}$ \\
\hline 267 & $3: 55: 34.95$ & $+53: 19: 10.6$ & 13.55 & 4.05 & 3.45 & 2.33 & 0.90 & 0.46 & 0.89 & k3 V: \\
\hline 268 & $3: 55: 35.68$ & $+53: 47: 37.0$ & 14.47 & 3.58 & 2.70 & 1.93 & 0.92 & 0.33 & 0.81 & f7 IV: \\
\hline 269 & $3: 55: 35.69$ & $+53: 18: 23.3$ & 12.96 & 3.15 & 2.28 & 1.33 & 0.63 & 0.23 & 0.52 & a3 IV \\
\hline 270 & $3: 55: 35.80$ & $+54: 02: 15.9$ & 12.78 & 3.33 & 2.59 & 1.85 & 0.85 & 0.32 & 0.76 & f6 IV \\
\hline 271 & $3: 55: 35.85$ & $+54: 10: 35.1$ & 16.72 & & & 2.48 & 1.06 & 0.47 & 0.96 & k0 IV \\
\hline 272 & $3: 55: 36.15$ & $+53: 43: 57.0$ & 16.77 & & & 2.65: & 1.17 & 0.50 & 1.03 & $\mathrm{k} 0 \mathrm{~V}$ \\
\hline 273 & $3: 55: 36.47$ & $+53: 10: 11.1$ & 16.13 & & & 2.50 & 0.99 & 0.55 & 0.95 & $\mathrm{k} 4.2 \mathrm{~V}$ \\
\hline 274 & $3: 55: 37.42$ & $+54: 00: 29.5$ & 13.98 & 3.28 & 2.38 & 1.59 & 0.77 & 0.28 & 0.65 & a6 IV: \\
\hline 275 & $3: 55: 38.35$ & $+53: 41: 38.1$ & 12.23 & 3.33 & 2.44 & 1.48 & 0.72 & 0.26 & 0.61 & a6 IV \\
\hline 276 & $3: 55: 38.48$ & $+54: 13: 05.7$ & 16.23 & & & 3.23: & 1.28 & 0.79 & 1.31 & $\mathrm{k} 5.5 \mathrm{~V}$ \\
\hline 277 & $3: 55: 38.69$ & $+53: 15: 39.7$ & 14.16 & 3.64 & 2.74 & 2.19 & 1.21 & 0.43 & 1.01 & b \\
\hline 278 & $3: 55: 38.72$ & $+53: 46: 41.0$ & 15.60 & & & 2.38 & 1.04 & 0.46 & 0.94 & $\mathrm{k} 0 \mathrm{~V}$ \\
\hline $279^{*}$ & $3: 55: 39.56$ & $+53: 52: 40.6$ & 9.47 & 2.26 & 1.88 & 1.22 & 0.54 & 0.24 & 0.50 & f7 V: \\
\hline 280 & $3: 55: 39.86$ & $+53: 13: 29.1$ & 13.67 & 2.97 & 2.29 & 1.63 & 0.74 & 0.28 & 0.69 & $\mathrm{f} 4 \mathrm{~V}$ \\
\hline 281 & $3: 55: 41.94$ & $+54: 01: 08.4$ & 16.33 & & & 2.83 & 1.24 & 0.53 & 1.20 & $\mathrm{k} * *$ \\
\hline 282 & $3: 55: 42.52$ & $+54: 06: 23.7$ & 13.97 & 3.29 & 2.41 & 1.65 & 0.80 & 0.30 & 0.69 & a5 III \\
\hline 283 & $3: 55: 43.02$ & $+53: 33: 00.5$ & 15.56 & & & 3.11 & 1.32 & 0.53 & 1.21 & $\mathrm{k} 1.2$ III \\
\hline 284 & $3: 55: 43.63$ & $+53: 43: 51.8$ & 12.63 & & 3.05 & 2.18 & 0.67 & 0.35 & 0.93 & k5 V: \\
\hline 285 & $3: 55: 45.60$ & $+53: 36: 09.7$ & 15.71 & & & 3.58 & 1.65 & 0.60 & 1.49 & k0 III \\
\hline 286 & $3: 55: 45.88$ & $+54: 10: 54.8$ & 15.39 & & & 3.61 & 1.59 & 0.67 & 1.34 & $\mathrm{k} 1.2$ III \\
\hline 287 & $3: 55: 46.26$ & $+53: 40: 43.5$ & 15.61 & & & 2.91 & 1.26 & 0.50 & 1.11 & k0.5 III \\
\hline 288 & $3: 55: 47.77$ & $+53: 09: 38.1$ & 12.11 & 2.93 & 2.40 & 1.66 & 0.73 & 0.27 & 0.69 & g1.5 V \\
\hline 289 & $3: 55: 48.28$ & $+53: 27: 14.6$ & 15.19 & 3.44: & 2.64 & 1.96 & 0.89 & 0.33 & 0.81 & f9 IV \\
\hline 290 & $3: 55: 48.52$ & $+53: 42: 19.2$ & 13.23 & 3.53 & 2.70 & 1.92 & 0.89 & 0.32 & 0.80 & f6 III \\
\hline 291 & $3: 55: 48.58$ & $+53: 31: 43.3$ & 14.99 & 3.79 & 2.87 & 2.08 & 0.98 & 0.36 & 0.88 & f6 III \\
\hline 292 & $3: 55: 48.87$ & $+53: 30: 14.8$ & 15.30 & & & 2.94 & 1.22 & 0.57 & 0.87 & $\mathrm{k} 2.7 \mathrm{~V}:$ \\
\hline 293 & $3: 55: 49.13$ & $+53: 16: 14.5$ & 13.69 & 3.15 & 2.31 & 1.35 & 0.64 & 0.23 & 0.50 & a5 IV \\
\hline 294 & $3: 55: 49.25$ & $+53: 13: 24.5$ & 12.82 & 3.03 & 2.18 & 1.26 & 0.58 & 0.21 & 0.50 & a7 III-V \\
\hline 295 & $3: 55: 49.95$ & $+53: 25: 26.1$ & 14.46 & 3.60 & 2.91 & 2.09 & 0.91 & 0.37 & 0.83 & g3 IV \\
\hline 296 & $3: 55: 50.50$ & $+53: 04: 41.4$ & 16.30 & & & 2.37 & 0.94 & 0.46 & 0.95 & $\mathrm{k} 2 \mathrm{~V}$ \\
\hline
\end{tabular}


Table 3. Continued

\begin{tabular}{|c|c|c|c|c|c|c|c|c|c|c|}
\hline No. & $\begin{array}{l}\mathrm{RA}(\mathrm{J} 2000) \\
\mathrm{h} \mathrm{m} \text { s }\end{array}$ & $\begin{array}{c}\operatorname{DEC}(\mathrm{J} 2000) \\
\circ, \prime \prime\end{array}$ & $\begin{array}{c}V \\
\text { mag }\end{array}$ & $\begin{array}{l}U-V \\
\operatorname{mag}\end{array}$ & $\begin{array}{l}P-V \\
\text { mag }\end{array}$ & $\begin{array}{l}X-V \\
\text { mag }\end{array}$ & $\begin{array}{l}Y-V \\
\operatorname{mag}\end{array}$ & $\begin{array}{l}Z-V \\
\operatorname{mag}\end{array}$ & $\begin{array}{l}V-S \\
\operatorname{mag}\end{array}$ & $\begin{array}{l}\text { Photom. } \\
\text { sp. type }\end{array}$ \\
\hline 297 & $3: 55: 50.69$ & $+54: 09: 40.7$ & 14.75 & 3.62 : & 2.76 & 1.99 & 0.95 & $0.37:$ & 0.85 & $\mathrm{f} * *$ \\
\hline 298 & $3: 55: 51.20$ & $+53: 45: 21.8$ & 15.79 & & & 2.77 & 1.09 & 0.54 & 1.03 & $\mathrm{k} 2.7 \mathrm{~V}$ \\
\hline 299 & $3: 55: 51.34$ & $+53: 55: 51.5$ & 13.74 & 4.17 & 2.99 & 1.93 & 0.95 & 0.33 & 0.79 & a2 III \\
\hline 300 & $3: 55: 51.98$ & $+53: 05: 00.8$ & 15.11 & 3.55 & 2.64 & 1.96 & 0.91 & 0.34 & 0.85 & f7 IV \\
\hline 301 & $3: 55: 52.38$ & $+53: 06: 43.6$ & 14.57 & 3.20 & 2.39 & 1.73 & 0.81 & 0.32 & 0.72 & f4 IV \\
\hline 302 & $3: 55: 52.51$ & $+53: 37: 35.7$ & 16.69 & & & 2.74 & 1.13 & $0.53:$ & 1.11 & $\mathrm{k} 2.5 \mathrm{~V}$ \\
\hline 303 & $3: 55: 52.56$ & $+53: 37: 51.2$ & 13.87 & 3.67 & 2.75 & 2.00 & 0.95 & 0.35 & 0.82 & f7 III \\
\hline 304 & $3: 55: 52.62$ & $+53: 29: 18.8$ & 14.58 & 4.26 & 3.36 & 2.45 & 1.10 & 0.41 & 1.01 & g1.5 III \\
\hline 305 & $3: 55: 52.69$ & $+53: 38: 05.8$ & 14.30 & 4.41: & 3.57 & 2.60 & 1.13 & 0.43 & 1.06 & g5 III \\
\hline 306 & $3: 55: 53.09$ & $+53: 43: 00.7$ & 14.78 & 3.60: & 2.76 & 2.02 & 0.95 & 0.35 & 0.82 & f6 IV \\
\hline 307 & $3: 55: 53.15$ & $+53: 27: 05.8$ & 13.93 & 3.66 & 2.74 & 1.92 & 0.90 & 0.33 & 0.79 & f5 III \\
\hline 308 & $3: 55: 53.46$ & $+53: 40: 54.8$ & 15.07 & 3.51: & 2.79 & 2.10 & 0.95 & 0.37 & 0.87 & $\mathrm{~g} 1 \mathrm{~V}$ \\
\hline 309 & $3: 55: 53.79$ & $+53: 18: 04.9$ & 14.09 & 3.24 & 2.43 & 1.70 & 0.80 & 0.30 & 0.74 & f3 IV \\
\hline 310 & $3: 55: 54.90$ & $+53: 04: 46.8$ & 16.77 & & & 2.71: & 1.03 & 0.54 & 1.00: & $\mathrm{k} * *$ \\
\hline 311 & $3: 55: 55.16$ & $+53: 48: 20.9$ & 14.30 & 3.33 & 2.59 & 1.89 & 0.88 & 0.32 & 0.79 & f8 IV \\
\hline 312 & $3: 55: 55.27$ & $+54: 06: 59.6$ & 14.23 & 3.49 & 2.64 & 1.88 & 0.90 & 0.34 & 0.79 & f3 IV \\
\hline 313 & $3: 55: 55.48$ & $+54: 13: 01.5$ & 14.66 & 3.44 & 2.69 & 1.98 & 0.93 & 0.35 & 0.80 & f7 V \\
\hline 314 & $3: 55: 55.66$ & $+54: 06: 36.5$ & 15.85 & & & 2.39 & 0.98 & 0.44 & 0.93 & $\mathrm{k} * *$ \\
\hline 315 & $3: 55: 55.81$ & $+53: 14: 12.4$ & 15.71 & & & 3.50 & 1.51 & 0.58 & 1.43 & $\mathrm{k} 2$ III \\
\hline 316 & $3: 55: 55.88$ & $+54: 13: 34.5$ & 14.14 & & 4.12 & 2.97 & 1.28 & 0.51 & 1.12 & k0 III \\
\hline 317 & $3: 55: 57.53$ & $+53: 14: 00.4$ & 14.73 & 4.13 & 2.94 & 2.10 & 1.00 & 0.34 & 0.96 & f3 III \\
\hline 318 & $3: 55: 57.58$ & $+54: 07: 18.5$ & 16.43 & & & 2.35 & 1.01 & 0.44 & 0.91 & $\mathrm{k} 0.7 \mathrm{~V}$ \\
\hline 319 & $3: 55: 59.82$ & $+53: 13: 33.2$ & 15.76 & & & 3.48 & 1.57 & 0.61 & 1.41 & $\mathrm{k} * *$ \\
\hline 320 & $3: 56: 00.94$ & $+54: 04: 30.6$ & 13.38 & 3.06 & 2.34 & 1.62 & 0.77 & 0.29 & 0.68 & f4 IV \\
\hline 321 & $3: 56: 01.14$ & $+54: 08: 39.2$ & 15.01 & 3.38 & 2.61 & 1.84 & 0.87 & 0.30 & 0.77 & f4 IV \\
\hline 322 & $3: 56: 01.18$ & $+53: 48: 26.9$ & 15.59 & & & 2.51 & 1.05 & 0.46 & 0.94 & $\mathrm{k} * *$ \\
\hline 323 & $3: 56: 01.42$ & $+53: 23: 07.5$ & 13.94 & 3.23 & 2.42 & 1.57 & 0.71 & 0.28 & 0.60 & a9 IV \\
\hline 324 & $3: 56: 01.73$ & $+53: 06: 07.8$ & 13.44 & 3.20 & 2.45 & 1.74 & 0.81 & 0.31 & 0.75 & f4 IV \\
\hline 325 & $3: 56: 05.77$ & $+53: 19: 50.7$ & 15.62 & 3.22 & 2.51 & 1.91 & 0.88 & 0.32 & 0.76 & g0 V \\
\hline 326 & $3: 56: 05.96$ & $+53: 04: 43.7$ & 15.35 & & & 4.05: & 1.86 & 0.71 & 1.67 & $\mathrm{k} 1.5$ III \\
\hline 327 & $3: 56: 06.21$ & $+54: 15: 29.0$ & 13.95 & 3.40 & 2.49 & 1.65 & 0.79 & 0.29 & 0.65 & f2 III \\
\hline 328 & $3: 56: 06.64$ & $+54: 01: 44.7$ & 15.07 & 3.28 & 2.52 & 1.89 & 0.87 & 0.33 & 0.79 & f8 $\mathrm{V}$ \\
\hline 329 & $3: 56: 06.68$ & $+53: 16: 17.3$ & 14.57 & 3.45 & 2.40 & 1.60 & 0.84 & 0.31 & 0.67 & b9 III \\
\hline 330 & $3: 56: 07.19$ & $+53: 26: 07.1$ & 13.36 & 3.44 & 2.61 & 1.86 & 0.88 & 0.32 & 0.79 & f4 III \\
\hline 331 & $3: 56: 07.45$ & $+53: 21: 21.5$ & 12.11 & 2.90 & 2.28 & 1.57 & 0.71 & 0.26 & 0.67 & f8 IV \\
\hline 332 & $3: 56: 08.14$ & $+53: 10: 10.1$ & 9.13 & 6.08 & 5.25 & 3.71 & 1.40 & 0.66 & 1.26 & $\mathrm{k} 8$ III \\
\hline 333 & $3: 56: 08.45$ & $+53: 17: 30.1$ & 14.32 & 3.21 & 2.34 & 1.57 & 0.77 & 0.31 & 0.59 & a3 III \\
\hline 334 & $3: 56: 08.55$ & $+53: 04: 15.7$ & 12.80 & 2.98 & 2.14 & 1.19 & 0.55 & 0.20 & 0.44 & $\mathrm{a} 3 \mathrm{~V}$ \\
\hline 335 & $3: 56: 08.74$ & $+53: 26: 16.4$ & 14.81 & 3.87 & 3.08 & 2.22 & 0.99 & 0.39 & 0.94 & g1.5 III \\
\hline 336 & $3: 56: 09.17$ & $+53: 29: 45.8$ & 14.32 & 3.69 & 2.96 & 2.17 & 0.95 & 0.38 & 0.90 & $\mathrm{~g} * *$ \\
\hline 337 & $3: 56: 11.11$ & $+54: 05: 29.0$ & 13.32 & 3.96 & 3.46 & 2.37 & 0.84 & 0.52 & 0.88 & $\mathrm{k} 3.7 \mathrm{~V}$ : \\
\hline 338 & $3: 56: 11.29$ & $+53: 11: 47.3$ & 14.75 & 3.10 & 2.37 & 1.90 & 1.03 & 0.39 & 0.84 & b8 V: \\
\hline 339 & $3: 56: 11.87$ & $+53: 56: 37.2$ & 16.50 & & & 2.70 & 1.13 & 0.44 & 1.06 & k0 III \\
\hline 340 & $3: 56: 12.02$ & $+53: 38: 18.0$ & 16.63 & & & 2.43 & 1.00 & 0.35 & 0.96 & $\mathrm{k}$ \\
\hline 341 & $3: 56: 12.06$ & $+53: 45: 22.1$ & 14.84 & & & 3.12 & 1.13 & 0.67 & 0.95 & $\mathrm{k} 7 \mathrm{~V}$ \\
\hline 342 & $3: 56: 12.61$ & $+53: 13: 53.1$ & 15.57 & 3.16 & 2.50 & 1.82 & 0.83 & 0.31 & 0.72 & f9 $\mathrm{V}$ \\
\hline 343 & $3: 56: 13.31$ & $+53: 18: 43.3$ & 14.12 & 3.10 & 2.27 & 1.54 & 0.71 & 0.26 & 0.66 & f1 IV \\
\hline 344 & $3: 56: 14.67$ & $+53: 09: 14.0$ & 14.13 & 3.15 & 2.29 & 1.47 & 0.68 & 0.26 & 0.60 & f2 III \\
\hline 345 & $3: 56: 14.71$ & $+53: 26: 35.8$ & 13.77 & 3.53 & 2.65 & 1.87 & 0.87 & 0.33 & 0.78 & f6 III \\
\hline 346 & $3: 56: 15.48$ & $+53: 55: 27.4$ & 14.50 & 4.06: & 3.11 & 2.28 & 1.04 & 0.37 & 0.95 & f9 IV \\
\hline 347 & $3: 56: 16.34$ & $+53: 16: 30.5$ & 12.45 & 3.23 & 2.36 & 1.47 & 0.70 & 0.27 & 0.61 & $\mathrm{a} * *$ \\
\hline 348 & $3: 56: 18.15$ & $+54: 13: 55.7$ & 13.93 & 3.58 & 2.85 & 2.06 & 0.95 & 0.36 & 0.83 & f9 IV \\
\hline 349 & $3: 56: 18.43$ & $+53: 28: 00.7$ & 14.15 & 3.74 & 2.98 & 2.15 & 0.97 & 0.36 & 0.90 & g2.5 III \\
\hline 350 & $3: 56: 18.50$ & $+53: 59: 08.1$ & 14.69 & 3.45 & 2.59 & 1.89 & 0.89 & 0.33 & 0.80 & f7 IV \\
\hline 351 & $3: 56: 18.82$ & $+53: 07: 00.6$ & 13.90 & 3.39 & 2.56 & 1.83 & 0.85 & 0.33 & 0.79 & f4 III \\
\hline 352 & $3: 56: 20.04$ & $+53: 16: 03.4$ & 15.60 & & & 2.48 & 0.98 & 0.54 & 0.96 & $\mathrm{k} 3.5 \mathrm{~V}$ \\
\hline 353 & $3: 56: 20.19$ & $+53: 30: 16.0$ & 16.00 & & & 2.82 & 1.19 & 0.48 & 1.13 & $\mathrm{k} 0.5 \mathrm{IV}$ \\
\hline 354 & $3: 56: 20.33$ & $+54: 13: 15.3$ & 15.18 & & & 3.38 & 1.53 & 0.61 & 1.34 & $\mathrm{k} 0.5 \mathrm{IV}$ \\
\hline 355 & $3: 56: 20.66$ & $+54: 03: 59.7$ & 15.60 & & & 2.95 & 1.20 & 0.64 & 1.25 & $\mathrm{k} 4 \mathrm{~V}$ \\
\hline 356 & $3: 56: 21.68$ & $+53: 11: 26.5$ & 12.38 & 2.82 & 2.23 & 1.57 & 0.71 & 0.27 & 0.68 & f6 $\mathrm{V}$ \\
\hline 357 & $3: 56: 22.76$ & $+53: 15: 35.4$ & 15.11 & 3.12 & 2.34 & 1.67 & 0.79 & 0.30 & 0.71 & f4 IV \\
\hline 358 & $3: 56: 22.95$ & $+53: 42: 47.0$ & 16.46 & & & 2.85 & 1.07 & 0.56 & 1.02 & k1 IV \\
\hline
\end{tabular}


Table 3. Continued

\begin{tabular}{|c|c|c|c|c|c|c|c|c|c|c|}
\hline No. & $\begin{array}{c}\text { RA (J2000) } \\
\text { h m s }\end{array}$ & $\begin{array}{c}\mathrm{DEC}(\mathrm{J} 2000) \\
\circ, \prime \prime\end{array}$ & $\begin{array}{c}V \\
\text { mag }\end{array}$ & $\begin{array}{l}U-V \\
\text { mag }\end{array}$ & $\begin{array}{l}P-V \\
\text { mag }\end{array}$ & $\begin{array}{l}X-V \\
\text { mag }\end{array}$ & $\begin{array}{l}Y-V \\
\text { mag }\end{array}$ & $\begin{array}{l}Z-V \\
\text { mag }\end{array}$ & $\begin{array}{l}V-S \\
\text { mag }\end{array}$ & $\begin{array}{l}\text { Photom. } \\
\text { sp. type }\end{array}$ \\
\hline 359 & $3: 56: 23.14$ & $+53: 47: 56.6$ & 16.00 & & & 3.03 & 1.11 & 0.65 & 0.95 & $\mathrm{k} 7 \mathrm{~V}$ \\
\hline 360 & $3: 56: 24.83$ & $+53: 45: 21.8$ & 14.07 & & 3.02 & 2.15 & 0.88 & 0.39 & 0.84 & $\mathrm{k} 0 \mathrm{~V}$ \\
\hline 361 & $3: 56: 25.20$ & $+53: 47: 36.7$ & 14.83 & 3.47 & 2.69 & 1.98 & 0.89 & 0.32 & 0.80 & f8 IV \\
\hline 362 & $3: 56: 25.28$ & $+53: 35: 55.3$ & 11.73 & $7.21:$ & 6.00 & 4.34 & 1.84 & 0.71 & 1.64 & $\mathrm{k}$ \\
\hline 363 & $3: 56: 26.15$ & $+53: 23: 09.5$ & 12.76 & 3.71 & 2.65 & 1.61 & 0.80 & 0.30 & 0.63 & a2 III \\
\hline 364 & $3: 56: 26.21$ & $+54: 15: 33.6$ & 11.72 & 3.51 & 2.47 & 1.43 & 0.72 & 0.26 & 0.60 & a1.5 III \\
\hline 365 & $3: 56: 28.04$ & $+53: 33: 06.3$ & 14.24 & 3.79 & 2.92 & 2.19 & 1.01 & 0.37 & 0.92 & $\mathrm{~g} * *$ \\
\hline 366 & $3: 56: 28.12$ & $+53: 38: 29.5$ & 16.52 & & & 2.45 & 1.11 & 0.39 & 0.99 & $\mathrm{~g}$ \\
\hline 367 & $3: 56: 28.40$ & $+53: 41: 08.1$ & 12.78 & 3.39 & 2.46 & 1.53 & 0.72 & 0.27 & 0.63 & a8 III \\
\hline 368 & $3: 56: 30.81$ & $+53: 34: 22.9$ & 14.86 & 3.98 & 2.84 & 1.88 & 0.88 & 0.31 & 0.77 & $\mathrm{f}$ \\
\hline 369 & $3: 56: 34.26$ & $+53: 33: 45.6$ & 14.62 & 3.87: & 3.03 & 2.25 & 1.03 & 0.40 & 0.97 & g1 IV \\
\hline 370 & $3: 56: 34.90$ & $+53: 57: 38.7$ & 14.59 & 3.87: & 2.92 & 2.13 & 1.01 & 0.37 & 0.90 & f4 III \\
\hline 371 & $3: 56: 35.54$ & $+53: 30: 34.6$ & 15.38 & & & 2.57 & 1.08 & 0.47 & 1.03 & $\mathrm{k} 1.2 \mathrm{~V}$ \\
\hline 372 & $3: 56: 35.56$ & $+53: 08: 33.9$ & 15.73 & & & $3.57:$ & 1.62 & 0.63 & 1.49 & k0.5 III \\
\hline 373 & $3: 56: 36.24$ & $+54: 10: 26.9$ & 14.01 & 3.55 & 2.64 & 1.89 & 0.91 & 0.33 & 0.79 & f3 IV \\
\hline 374 & $3: 56: 36.50$ & $+53: 09: 30.1$ & 15.11 & 3.55 & 2.78 & 1.99 & 0.91 & 0.35 & 0.83 & g1.5 III \\
\hline 375 & $3: 56: 36.80$ & $+54: 00: 56.9$ & 12.40 & 3.35 & 2.51 & 1.55 & 0.71 & 0.25 & 0.61 & a8 IV \\
\hline 376 & $3: 56: 37.55$ & $+54: 00: 41.3$ & 16.07 & & & 2.45 & 1.07 & 0.37 & 1.03 & g5 \\
\hline 377 & $3: 56: 38.97$ & $+53: 23: 20.3$ & 13.98 & 3.31 & 2.38 & 1.55 & 0.72 & 0.27 & 0.61 & fO III \\
\hline 378 & $3: 56: 39.98$ & $+53: 23: 54.0$ & 12.24 & 2.68 & 1.95 & 1.16 & 0.59 & 0.21 & 0.49 & b8 III \\
\hline 379 & $3: 56: 40.87$ & $+54: 07: 14.2$ & 15.58 & & & 2.86 & 1.24 & 0.50 & 1.12 & k0 IV \\
\hline 380 & $3: 56: 40.97$ & $+54: 07: 50.3$ & 16.22 & & & 2.48 & 1.08 & 0.47 & 0.94 & $\mathrm{k} 0.5 \mathrm{~V}$ \\
\hline 381 & $3: 56: 41.47$ & $+53: 20: 27.3$ & 14.85 & & & 4.24 & 1.79 & 0.75 & 1.56 & k4.2 III \\
\hline 382 & $3: 56: 41.52$ & $+53: 46: 47.9$ & 16.11 & & & 2.51 & 1.04 & 0.45 & 1.00 & $\mathrm{k} 0.5 \mathrm{~V}$ \\
\hline 383 & $3: 56: 41.96$ & $+53: 22: 11.4$ & 14.46 & & & 4.67 & 1.95 & 0.84 & 1.76 & $\mathrm{k}-\mathrm{m}$ \\
\hline 384 & $3: 56: 42.71$ & $+53: 15: 06.4$ & 14.55 & 3.11 & 2.29 & 1.56 & 0.73 & 0.28 & 0.65 & f2 IV \\
\hline 385 & $3: 56: 43.65$ & $+54: 05: 23.4$ & 15.15 & 3.69 & 2.71 & 1.98 & 0.92 & 0.36 & 0.80 & f5 III \\
\hline 386 & $3: 56: 43.74$ & $+53: 21: 22.3$ & 14.93 & 3.36: & 2.41 & 1.60 & 0.81 & 0.35 & 0.73 & a2 $\mathrm{V}$ \\
\hline 387 & $3: 56: 43.90$ & $+53: 22: 41.9$ & 12.36 & 3.36 & 2.48 & 1.59 & 0.76 & 0.25 & 0.74 & a9 III \\
\hline 388 & $3: 56: 44.26$ & $+53: 45: 35.5$ & 13.44 & 3.40 & 2.54 & 1.71 & 0.82 & 0.30 & 0.69 & f2 IV \\
\hline 389 & $3: 56: 44.91$ & $+54: 15: 48.3$ & 14.90 & & & 4.06: & 1.81 & 0.74 & 1.53 & k2 III \\
\hline 390 & $3: 56: 45.00$ & $+54: 15: 19.4$ & 13.10 & 3.28 & 2.47 & 1.74 & 0.84 & 0.29 & 0.74 & f4 III \\
\hline $391^{*}$ & $3: 56: 47.41$ & $+53: 33: 36.8$ & 10.92 & 4.46 & 3.77 & 2.73 & 1.09 & 0.60 & 1.23 & $\mathrm{~m}-\mathrm{k}$ \\
\hline 392 & $3: 56: 48.15$ & $+53: 10: 17.4$ & 15.08 & 3.40 & 2.65 & 1.93 & 0.87 & 0.33 & 0.80 & g1.5 III \\
\hline 393 & $3: 56: 48.35$ & $+53: 42: 03.2$ & 15.14 & 3.58 & 2.70 & 2.02 & 0.94 & 0.34 & 0.85 & $\mathrm{f} * *$ \\
\hline 394 & $3: 56: 49.29$ & $+53: 59: 18.3$ & 14.54 & 4.22 & 3.28 & 2.43 & 1.10 & 0.40 & 1.00 & g1.5 III \\
\hline 395 & $3: 56: 49.95$ & $+53: 19: 51.4$ & 13.51 & 3.21 & 2.43 & 1.71 & 0.81 & 0.30 & 0.72 & f4 IV \\
\hline 396 & $3: 56: 50.40$ & $+53: 18: 45.0$ & 14.18 & 4.24: & 3.39 & 2.45 & 1.10 & 0.43 & 1.00 & g6 ** \\
\hline 397 & $3: 56: 50.45$ & $+53: 54: 31.9$ & 12.91 & 3.55 & 2.50 & 1.55 & 0.78 & 0.27 & 0.66 & a0 III \\
\hline 398 & $3: 56: 50.65$ & $+53: 25: 48.1$ & 14.40 & 3.78 & 2.95 & 2.13 & 0.96 & 0.35 & 0.88 & g1.5 III \\
\hline 399 & $3: 56: 50.68$ & $+53: 16: 48.5$ & 15.21 & & & 2.66 & 1.05 & 0.56 & 1.02 & $\mathrm{k} 3.5 \mathrm{~V}$ \\
\hline 400 & $3: 56: 50.76$ & $+54: 13: 34.1$ & 16.31 & & & 3.05: & 1.19 & 0.67 & 1.18 & $\mathrm{k} 5 \mathrm{~V}$ \\
\hline 401 & $3: 56: 51.12$ & $+53: 07: 35.9$ & 14.84 & 3.38 & 2.67 & 1.96 & 0.89 & 0.36 & 0.86 & $\mathrm{~g} * *$ \\
\hline 402 & $3: 56: 51.25$ & $+53: 21: 08.3$ & 16.45 & & & 2.60 & 1.14 & 0.52 & 1.08 & $\mathrm{k} 1 \mathrm{~V}$ \\
\hline 403 & $3: 56: 52.35$ & $+53: 23: 54.7$ & 11.66 & 3.01 & 2.17 & 1.23 & 0.61 & 0.22 & 0.49 & $\mathrm{a} 0 \mathrm{IV}$ \\
\hline $404 *$ & $3: 56: 52.79$ & $+53: 16: 40.8$ & 13.89 & 2.55 & 1.88 & 1.79 & 0.53 & -0.11 & 0.84 & $\mathrm{a}$ \\
\hline 405 & $3: 56: 54.05$ & $+53: 46: 00.3$ & 12.56 & 3.66 & 2.93 & 2.06 & 0.93 & 0.34 & 0.85 & g2.5 III \\
\hline 406 & $3: 56: 54.40$ & $+54: 04: 09.6$ & 14.28 & 4.28: & 2.90 & 1.93 & 1.03 & 0.38 & 0.83 & a0 IV-V \\
\hline 407 & $3: 56: 55.30$ & $+53: 22: 09.1$ & 14.12 & 3.47 & 2.61 & 2.12 & 1.16 & 0.42 & 0.95 & b6 IV-V: \\
\hline 408 & $3: 56: 56.25$ & $+53: 10: 41.8$ & 14.06 & 3.42 & 2.72 & 1.96 & 0.87 & 0.34 & 0.82 & g1 IV \\
\hline 409 & $3: 56: 57.56$ & $+53: 16: 18.4$ & 14.62 & & & 3.19 & 1.32 & 0.57 & 1.21 & $\mathrm{k} 1.5 \mathrm{III}$ \\
\hline 410 & $3: 56: 57.70$ & $+53: 04: 57.0$ & 14.97 & 3.98 & 3.32 & 2.33 & 0.89 & 0.45 & 0.89 & $\mathrm{k} 2.7 \mathrm{~V}:$ \\
\hline 411 & $3: 56: 58.14$ & $+53: 26: 12.4$ & 16.21 & & & 2.85 & 1.20 & 0.50 & 1.11 & k0.7 IV \\
\hline 412 & $3: 56: 59.45$ & $+54: 14: 33.1$ & 15.31 & 3.50 & 2.70 & 1.92 & 0.94 & 0.34 & 0.78 & f4 IV \\
\hline 413 & $3: 56: 59.66$ & $+53: 23: 51.1$ & 16.05 & & & 2.48 & 1.09 & 0.50 & 1.00 & $\mathrm{k} 0.5 \mathrm{~V}$ \\
\hline 414 & $3: 57: 00.56$ & $+53: 59: 24.5$ & 14.09 & 3.64 & 2.78 & 2.04 & 0.95 & 0.35 & 0.86 & f8 IV \\
\hline 415 & $3: 57: 00.59$ & $+54: 11: 10.9$ & 14.31 & 3.38 & 2.48 & 1.81 & 0.97 & 0.36 & 0.78 & b6 IV: \\
\hline 416 & $3: 57: 00.95$ & $+53: 48: 18.5$ & 12.85 & 3.33 & 2.44 & 1.43 & 0.69 & 0.24 & 0.54 & $\mathrm{a} 3 \mathrm{~V}$ \\
\hline 417 & $3: 57: 00.96$ & $+53: 18: 29.8$ & 13.22 & 3.14 & 2.42 & 1.72 & 0.80 & 0.29 & 0.73 & $\mathrm{f} 4 \mathrm{~V}$ \\
\hline 418 & $3: 57: 01.95$ & $+53: 23: 27.7$ & 12.28 & 2.80 & 2.00 & 1.12 & 0.57 & 0.21 & 0.45 & b9 $\mathrm{V}$ \\
\hline 419 & $3: 57: 02.58$ & $+53: 19: 24.7$ & 13.42 & 4.05 & 2.77 & 1.92 & 1.03 & 0.38 & 0.88 & b7 IV: \\
\hline 420 & $3: 57: 03.37$ & $+53: 16: 34.7$ & 14.45 & 3.42 & 2.49 & 1.79 & 0.86 & 0.33 & 0.79 & f5 IV \\
\hline
\end{tabular}


Table 3. Continued

\begin{tabular}{|c|c|c|c|c|c|c|c|c|c|c|}
\hline No. & $\begin{array}{c}\text { RA (J2000) } \\
\text { h m s s }\end{array}$ & $\begin{array}{c}\operatorname{DEC}(\mathrm{J} 2000) \\
\circ, \prime \prime\end{array}$ & $\begin{array}{c}V \\
\operatorname{mag}\end{array}$ & $\begin{array}{l}U-V \\
\text { mag }\end{array}$ & $\begin{array}{l}P-V \\
\text { mag }\end{array}$ & $\begin{array}{l}X-V \\
\text { mag }\end{array}$ & $\begin{array}{l}Y-V \\
\text { mag }\end{array}$ & $\begin{array}{l}Z-V \\
\text { mag }\end{array}$ & $\begin{array}{l}V-S \\
\text { mag }\end{array}$ & $\begin{array}{l}\text { Photom. } \\
\text { sp. type }\end{array}$ \\
\hline 421 & $3: 57: 03.53$ & $+53: 57: 00.5$ & 12.55 & 5.01 & 4.15 & 2.95 & 1.27 & 0.48 & 1.13 & g9.5 III \\
\hline 422 & $3: 57: 03.53$ & $+53: 52: 57.5$ & 14.03 & & 4.49: & 3.26 & 1.41 & 0.55 & 1.26 & k0 III \\
\hline 423 & $3: 57: 03.57$ & $+53: 18: 06.3$ & 15.12 & 3.72 & 3.05 & 2.16 & 0.95 & 0.36 & 0.88 & g5 IV \\
\hline 424 & $3: 57: 03.87$ & $+53: 42: 03.5$ & 16.81 & & & $2.46:$ & 1.00 & 0.47 & 0.95 & $\mathrm{k} 2 \mathrm{~V}$ \\
\hline 425 & $3: 57: 05.35$ & $+53: 03: 14.2$ & 13.52 & 2.91 & 2.21 & 1.56 & 0.71 & 0.28 & 0.58 & f4 IV \\
\hline 426 & $3: 57: 05.98$ & $+53: 03: 55.8$ & 16.66 & & & 2.14 & 0.93 & 0.44 & 0.78 & $\mathrm{~g} * *$ \\
\hline 427 & $3: 57: 06.02$ & $+53: 47: 34.3$ & 15.06 & 3.45: & 2.68 & 2.00 & 0.93 & 0.34 & 0.83 & f8 $\mathrm{V}$ \\
\hline 428 & $3: 57: 06.19$ & $+54: 13: 57.7$ & 12.38 & 3.31 & 2.47 & 1.60 & 0.76 & 0.28 & 0.64 & fo IV \\
\hline 429 & $3: 57: 06.45$ & $+53: 45: 26.3$ & 12.72 & 3.22 & 2.57 & 1.84 & 0.84 & 0.31 & 0.79 & f8 $\mathrm{V}$ \\
\hline 430 & $3: 57: 06.61$ & $+53: 25: 56.0$ & 12.06 & 3.93 & 2.97 & 2.04 & 0.94 & 0.34 & 0.86 & f3 III \\
\hline 431 & $3: 57: 06.83$ & $+53: 55: 35.4$ & 14.53 & 3.91: & 2.87 & 1.77 & 0.84 & 0.29 & 0.68 & a3 III \\
\hline 432 & $3: 57: 07.07$ & $+53: 39: 24.6$ & 16.74 & & & 2.62 & 1.07 & 0.47 & 1.04 & k0 IV \\
\hline 433 & $3: 57: 07.38$ & $+53: 45: 06.5$ & 14.85 & 4.03 & 3.39 & 2.41 & 1.01 & 0.43 & 0.97 & g9.5 III \\
\hline 434 & $3: 57: 07.67$ & $+53: 51: 51.3$ & 14.12 & 3.90 & 3.12 & 2.26 & 1.00 & 0.37 & 0.92 & g3 III \\
\hline 435 & $3: 57: 08.07$ & $+53: 40: 13.4$ & 15.01 & 3.75 & 2.98 & 2.15 & 0.95 & 0.36 & 0.87 & g2 III \\
\hline 436 & $3: 57: 08.44$ & $+54: 03: 52.7$ & 13.93 & & 4.40 & 3.16 & 1.37 & 0.53 & 1.22 & k0 III \\
\hline 437 & $3: 57: 09.22$ & $+54: 03: 25.7$ & 14.91 & $3.72:$ & 2.93 & 2.14 & 0.98 & 0.38 & 0.86 & f9.5 IV \\
\hline 438 & $3: 57: 10.15$ & $+53: 14: 09.1$ & 15.44 & & $3.28:$ & 2.31 & 0.96 & 0.43 & 0.92 & $\mathrm{k} 1 \mathrm{~V}$ \\
\hline 439 & $3: 57: 10.80$ & $+53: 20: 08.4$ & 15.09 & 3.74 & 2.90 & 1.98 & 0.90 & 0.34 & 0.83 & f6 III \\
\hline 440 & $3: 57: 11.21$ & $+53: 06: 56.1$ & 15.21 & 3.16: & 2.35 & 1.69 & 0.82 & 0.31 & 0.75 & a7 III \\
\hline 441 & $3: 57: 12.71$ & $+53: 27: 13.3$ & 13.43 & 3.87 & 2.80 & 1.70 & 0.81 & 0.26 & 0.66 & fo IV \\
\hline 442 & $3: 57: 12.86$ & $+53: 06: 23.3$ & 13.83 & 4.35 & 3.78 & 2.60 & 0.92 & 0.57 & 0.97 & k5 V: \\
\hline 443 & $3: 57: 14.56$ & $+53: 14: 54.7$ & 14.92 & 3.85 & 2.97 & 2.14 & 0.94 & 0.40 & 0.85 & g2 III \\
\hline 444 & $3: 57: 14.68$ & $+53: 11: 22.4$ & 15.30 & 3.42 & 2.57 & 2.03 & 1.03 & 0.37 & 0.91 & $\mathrm{a}$ \\
\hline 445 & $3: 57: 15.85$ & $+53: 59: 16.7$ & 15.94 & & & 3.15 & 1.17 & 0.67 & 1.21: & $\mathrm{k} * *$ \\
\hline 446 & $3: 57: 16.85$ & $+53: 26: 32.2$ & 16.21 & & & 2.97 & 1.33 & 0.60 & 1.28 & $\mathrm{k} 2 \mathrm{~V}$ \\
\hline 447 & $3: 57: 17.87$ & $+53: 14: 23.5$ & 14.90 & 3.59 & 2.59 & 1.79 & 0.94 & 0.35 & 0.76 & b8 III \\
\hline 448 & $3: 57: 18.32$ & $+53: 23: 24.5$ & 13.54 & 3.91 & 2.75 & 1.69 & 0.85 & 0.31 & 0.69 & a2 III \\
\hline 449 & $3: 57: 19.50$ & $+54: 13: 21.1$ & 14.73 & 3.74: & 2.98 & 2.21 & 0.99 & 0.37 & 0.90 & g1.5 IV \\
\hline 450 & $3: 57: 20.17$ & $+54: 10: 12.8$ & 14.85 & 3.31 & 2.49 & 1.98 & 1.08 & 0.41 & 0.88 & b8 V: \\
\hline 451 & $3: 57: 21.37$ & $+53: 33: 57.8$ & 12.66 & 5.48 & 4.59 & 3.21 & 1.33 & 0.53 & 1.19 & k2 III \\
\hline 452 & $3: 57: 21.89$ & $+53: 24: 53.4$ & 14.43 & & & 3.53 & 1.48 & 0.59 & 1.34 & k3 III \\
\hline 453 & $3: 57: 22.30$ & $+53: 41: 58.3$ & 13.70 & 3.39 & 2.41 & 1.63 & 0.85 & 0.31 & 0.72 & b8.5 III \\
\hline 454 & $3: 57: 22.30$ & $+53: 11: 21.2$ & 14.52 & 3.69 & 2.58 & 1.76 & 0.91 & 0.37 & 0.78 & b9 III \\
\hline 455 & $3: 57: 22.45$ & $+54: 05: 55.7$ & 14.91 & 3.64: & 2.63 & 1.93 & 0.93 & 0.34 & 0.79 & f6 IV \\
\hline 456 & $3: 57: 22.69$ & $+54: 02: 30.5$ & 15.63 & & & 2.36 & 0.99 & 0.43 & 0.91 & k0 IV \\
\hline 457 & $3: 57: 24.90$ & $+54: 10: 03.4$ & 11.70 & 3.39 & 2.49 & 1.49 & 0.69 & 0.25 & 0.57 & a6 III \\
\hline 458 & $3: 57: 25.11$ & $+53: 27: 55.1$ & 16.23 & & & 2.40 & 1.00 & 0.44 & 0.97 & $\mathrm{k} 0.7 \mathrm{~V}$ \\
\hline 459 & $3: 57: 25.47$ & $+53: 23: 24.0$ & 14.55 & 3.79 & 2.93 & 2.11 & 0.97 & 0.36 & 0.86 & f7 III \\
\hline 460 & $3: 57: 25.99$ & $+53: 19: 02.3$ & 16.56 & & & 2.65 & 1.02 & 0.53 & 1.01 & $\mathrm{k} 3.2 \mathrm{~V}$ \\
\hline 461 & $3: 57: 27.11$ & $+53: 20: 52.8$ & 14.16 & 3.33 & 2.53 & 1.79 & 0.85 & 0.32 & 0.74 & f3 III \\
\hline 462 & $3: 57: 27.81$ & $+53: 04: 47.5$ & 14.95 & $3.29:$ & 2.43 & 1.78 & 0.81 & 0.31 & 0.70 & f7 III \\
\hline 463 & $3: 57: 27.94$ & $+53: 36: 00.7$ & 16.67 & & & 2.43 & 1.04 & 0.34 & 0.99 & g-k \\
\hline 464 & $3: 57: 28.81$ & $+54: 09: 23.3$ & 13.48 & 3.66 & 2.69 & 1.79 & 0.84 & 0.31 & 0.72 & f1 III \\
\hline 465 & $3: 57: 29.48$ & $+53: 55: 06.2$ & 12.82 & 4.66 & 3.89 & 2.77 & 1.21 & 0.46 & 1.06 & g8.5 III \\
\hline 466 & $3: 57: 29.86$ & $+53: 37: 24.7$ & 13.55 & 3.19 & 2.44 & 1.72 & 0.80 & 0.29 & 0.72 & f3 III \\
\hline 467 & $3: 57: 31.49$ & $+53: 42: 04.7$ & 12.98 & 3.58 & 2.83 & 2.04 & 0.94 & 0.35 & 0.86 & f7 III \\
\hline 468 & $3: 57: 31.63$ & $+53: 32: 43.3$ & 13.90 & 3.97 & 3.16 & 2.29 & 1.03 & 0.39 & 0.95 & g3 III \\
\hline 469 & $3: 57: 32.51$ & $+53: 28: 52.6$ & 14.95 & 3.74 & 2.95 & 2.14 & 0.99 & 0.39 & 0.88 & f9 IV \\
\hline 470 & $3: 57: 32.61$ & $+53: 43: 01.1$ & 16.25 & & & 2.95 & 1.22 & 0.47 & 1.25 & $\mathrm{k}-\mathrm{m}$ \\
\hline 471 & $3: 57: 32.97$ & $+53: 20: 51.1$ & 16.44 & & & 2.29 & 0.95 & 0.43 & 0.90 & $\mathrm{k} 1 \mathrm{~V}$ \\
\hline 472 & $3: 57: 33.11$ & $+53: 19: 09.0$ & 15.19 & 3.46 & 2.60 & 1.88 & 0.86 & 0.32 & 0.78 & f7 IV \\
\hline 473 & $3: 57: 33.62$ & $+53: 48: 37.6$ & 11.62 & 3.09 & 2.29 & 1.27 & 0.60 & 0.22 & 0.48 & a $3 \mathrm{~V}$ \\
\hline 474 & $3: 57: 33.97$ & $+53: 04: 15.6$ & 13.36 & 3.10 & 2.45 & 1.76 & 0.79 & 0.29 & 0.73 & f8 $\mathrm{V}$ \\
\hline 475 & $3: 57: 34.09$ & $+53: 14: 10.1$ & 16.17 & & & 3.12 & 1.11 & 0.69 & 1.08 & $\mathrm{k} 7 \mathrm{~V}$ \\
\hline 476 & $3: 57: 34.19$ & $+53: 21: 49.9$ & 16.76 & & & 2.09 & 0.96 & 0.48 & 0.91 & $\mathrm{~g}$ \\
\hline 477 & $3: 57: 34.32$ & $+53: 46: 55.0$ & 14.59 & 3.80 & 2.90 & 2.02 & 0.95 & 0.34 & 0.82 & f5 III \\
\hline 478 & $3: 57: 34.39$ & $+53: 24: 41.2$ & 13.41 & 3.99 & 3.42 & 2.31 & 0.90 & 0.45 & 0.89 & k3 V: \\
\hline 479 & $3: 57: 34.53$ & $+53: 17: 03.4$ & 15.68 & & & 2.43 & 1.04 & 0.48 & 0.97 & $\mathrm{k} 1.5 \mathrm{~V}$ \\
\hline 480 & $3: 57: 34.93$ & $+53: 50: 31.6$ & 15.28 & 3.54 & 2.87 & 2.12 & 0.93 & 0.38 & 0.87 & g5 IV \\
\hline 481 & $3: 57: 34.97$ & $+53: 13: 02.2$ & 15.75 & & & $3.72:$ & 1.65 & 0.69 & 1.46 & $\mathrm{k} 1.5 \mathrm{III}$ \\
\hline 482 & $3: 57: 35.18$ & $+53: 13: 38.5$ & 16.06 & & & 2.46 & 0.99 & 0.36 & 1.02 & $g-k$ \\
\hline
\end{tabular}


Table 3. Continued

\begin{tabular}{|c|c|c|c|c|c|c|c|c|c|c|}
\hline No. & $\begin{array}{c}\mathrm{RA}(\mathrm{J} 2000) \\
\mathrm{h} \mathrm{m} \text { s }\end{array}$ & $\begin{array}{c}\mathrm{DEC}(\mathrm{J} 2000) \\
\circ, \prime \prime\end{array}$ & $\begin{array}{c}V \\
\operatorname{mag}\end{array}$ & $\begin{array}{l}U-V \\
\mathrm{mag}\end{array}$ & $\begin{array}{l}P-V \\
\mathrm{mag}\end{array}$ & $\begin{array}{l}X-V \\
\text { mag }\end{array}$ & $\begin{array}{l}Y-V \\
\operatorname{mag}\end{array}$ & $\begin{array}{l}Z-V \\
\operatorname{mag}\end{array}$ & $\begin{array}{l}V-S \\
\text { mag }\end{array}$ & $\begin{array}{l}\text { Photom. } \\
\text { sp. type }\end{array}$ \\
\hline 483 & $3: 57: 35.25$ & $+53: 41: 20.0$ & 14.02 & 3.95 & 3.15 & 2.30 & 1.03 & 0.40 & 0.93 & g3 III \\
\hline 484 & $3: 57: 35.38$ & $+54: 00: 46.3$ & 14.09 & & & 3.35 & 1.37 & 0.60 & 1.21 & k2.7 III \\
\hline 485 & $3: 57: 35.43$ & $+53: 22: 54.0$ & 14.93 & & 3.21 & 2.27 & 0.95 & 0.42 & 0.89 & $\mathrm{k} 0.5 \mathrm{~V}$ \\
\hline 486 & $3: 57: 35.60$ & $+54: 05: 44.4$ & 13.74 & 3.41 & 2.55 & 1.71 & 0.80 & 0.28 & 0.71 & $\mathrm{f} * *$ \\
\hline 487 & $3: 57: 35.83$ & $+53: 45: 52.7$ & 15.26 & 3.59 & 2.85 & 2.05 & 0.92 & 0.34 & 0.85 & g1 IV \\
\hline 488 & $3: 57: 36.04$ & $+53: 31: 18.3$ & 14.29 & & & 3.28 & 1.40 & 0.56 & 1.28 & $\mathrm{k} 1.2 \mathrm{III}$ \\
\hline 489 & $3: 57: 36.72$ & $+53: 03: 44.1$ & 14.66 & 3.34 & 2.62 & 1.89 & 0.86 & 0.33 & 0.76 & f7 III \\
\hline $490^{*}$ & $3: 57: 37.00$ & $+53: 40: 27.0$ & 10.72 & 2.52 & 1.87 & 1.07 & 0.55 & 0.20 & 0.45 & $\mathrm{~b} 8$ \\
\hline 491 & $3: 57: 37.02$ & $+53: 56: 54.8$ & 13.63 & 3.42 & 2.50 & 1.52 & 0.69 & 0.27 & 0.56 & f5 IV \\
\hline 492 & $3: 57: 37.76$ & $+53: 59: 08.9$ & 14.26 & 3.38 & 2.68 & 1.95 & 0.88 & 0.34 & 0.80 & f8 IV \\
\hline 493 & $3: 57: 38.29$ & $+54: 06: 49.6$ & 15.62 & & & 3.50 & 1.57 & 0.61 & 1.34 & k0 III \\
\hline 494 & $3: 57: 38.38$ & $+53: 08: 20.6$ & 13.20 & 3.73 & 3.11 & 2.15 & 0.85 & 0.41 & 0.85 & $\mathrm{k} 1.5 \mathrm{~V}$ \\
\hline 495 & $3: 57: 39.12$ & $+53: 52: 13.1$ & 15.17 & 3.56 & 2.84 & 2.08 & 0.92 & 0.36 & 0.85 & g2 IV \\
\hline 496 & $3: 57: 39.20$ & $+53: 29: 29.6$ & 16.38 & & & 2.39 & 1.08 & 0.50 & 0.95 & $\mathrm{~g} * *$ \\
\hline 497 & $3: 57: 40.20$ & $+53: 31: 15.2$ & 14.10 & 3.70 & 2.84 & 2.09 & 0.98 & 0.37 & 0.90 & f7 IV \\
\hline 498 & $3: 57: 40.30$ & $+54: 05: 36.6$ & 15.41 & & & 3.82 & 1.64 & 0.63 & 1.42 & k0.5 III \\
\hline 499 & $3: 57: 41.43$ & $+53: 11: 37.9$ & 16.06 & & & 2.35 & 1.00 & 0.45 & 0.97 & $\mathrm{k} * *$ \\
\hline 500 & $3: 57: 42.22$ & $+54: 01: 44.2$ & 13.54 & & 4.75 & 3.40 & 1.45 & 0.58 & 1.29 & k1.5 III \\
\hline 501 & $3: 57: 42.26$ & $+53: 40: 08.6$ & 15.22 & 3.66 & 2.68 & 1.97 & 0.94 & 0.34 & 0.83 & f6 IV \\
\hline 502 & $3: 57: 42.40$ & $+53: 18: 53.7$ & 14.43 & & & 3.39 & 1.42 & 0.60 & 1.28 & k2 III \\
\hline 503 & $3: 57: 42.44$ & $+53: 22: 02.7$ & 15.97 & & & 3.47 & 1.54 & 0.59 & 1.41 & k0.7 III \\
\hline 504 & $3: 57: 43.07$ & $+53: 30: 20.0$ & 16.32 & & & 3.04: & 1.32 & 0.53 & 1.19 & k0.7 IV \\
\hline 505 & $3: 57: 44.58$ & $+53: 24: 59.0$ & 11.73 & 2.93 & 2.14 & 1.17 & 0.56 & 0.20 & 0.45 & a2 IV \\
\hline 506 & $3: 57: 44.62$ & $+53: 47: 10.1$ & 16.56 & & & 2.51 & 1.12 & 0.50 & 0.93 & $\mathrm{k} 1 \mathrm{~V}$ \\
\hline 507 & $3: 57: 46.09$ & $+53: 22: 06.3$ & 14.81 & 3.25 & 2.48 & 1.83 & 0.84 & 0.33 & 0.79 & f6 $\mathrm{V}$ \\
\hline 508 & $3: 57: 46.17$ & $+53: 11: 56.3$ & 14.96 & & & 3.24 & 1.46 & 0.59 & 1.28 & k0 III \\
\hline 509 & $3: 57: 46.53$ & $+53: 43: 14.1$ & 14.90 & & & 2.71 & 1.10 & 0.50 & 1.05 & $\mathrm{k} 1 \mathrm{IV}$ \\
\hline 510 & $3: 57: 47.37$ & $+53: 26: 36.6$ & 13.38 & & & 4.09 & 1.66 & 0.74 & 1.49 & k6 III \\
\hline 511 & $3: 57: 47.45$ & $+53: 03: 05.3$ & 14.31 & 3.25 & 2.36 & 1.68 & 0.81 & 0.29 & 0.61 & f1 IV \\
\hline 512 & $3: 57: 47.47$ & $+53: 40: 24.5$ & 12.87 & 5.63 & 4.60 & 3.34 & 1.45 & 0.57 & 1.33 & k0.7 III \\
\hline 513 & $3: 57: 47.65$ & $+53: 21: 28.0$ & 14.09 & 3.23 & 2.44 & 1.74 & 0.80 & 0.31 & 0.74 & f5 IV \\
\hline 514 & $3: 57: 47.92$ & $+53: 58: 42.5$ & 16.85 & & & 2.31: & 1.00 & 0.46 & 0.89 & $\mathrm{k} 0.5 \mathrm{~V}$ \\
\hline 515 & $3: 57: 47.98$ & $+53: 56: 35.6$ & 12.31 & 2.90 & 2.10 & 1.14 & 0.56 & 0.19 & 0.42 & a1 V \\
\hline 516 & $3: 57: 48.11$ & $+53: 40: 45.2$ & 15.29 & & & 2.45 & 1.03 & 0.43 & 0.95 & k0 IV \\
\hline 517 & $3: 57: 49.28$ & $+54: 02: 45.0$ & 14.65 & 4.03 & 2.87 & 1.80 & 0.90 & 0.32 & 0.68 & a3 III \\
\hline 518 & $3: 57: 50.36$ & $+53: 17: 13.1$ & 14.28 & 3.07 & 2.13 & 1.38 & 0.74 & 0.28 & 0.59 & b7 IV \\
\hline 519 & $3: 57: 50.48$ & $+54: 11: 07.1$ & 12.80 & 3.26 & 2.46 & 1.68 & 0.83 & 0.30 & 0.65 & a4 III \\
\hline 520 & $3: 57: 51.59$ & $+53: 18: 58.9$ & 15.71 & & & $3.62:$ & 1.66 & 0.66 & 1.42 & k0.5 III \\
\hline 521 & $3: 57: 52.97$ & $+53: 53: 34.9$ & 16.85 & & & $2.42:$ & 0.96 & 0.42 & 0.90 & k0.7 IV \\
\hline 522 & $3: 57: 53.49$ & $+53: 28: 17.1$ & 15.59 & & & 3.50 & 1.57 & 0.60 & 1.44 & k0.5 III \\
\hline 523 & $3: 57: 53.52$ & $+53: 11: 05.7$ & 14.69 & 3.35 & 2.51 & 1.79 & 0.83 & 0.31 & 0.75 & f5 III \\
\hline 524 & $3: 57: 54.72$ & $+53: 30: 31.6$ & 14.45 & 3.76 & 2.76 & 1.87 & 0.90 & 0.34 & 0.76 & f2 III \\
\hline 525 & $3: 57: 56.60$ & $+53: 31: 28.9$ & 12.40 & 3.60 & 2.66 & 1.64 & 0.76 & 0.27 & 0.64 & f7 IV \\
\hline 526 & $3: 57: 57.58$ & $+53: 55: 17.1$ & 13.65 & 3.29 & 2.41 & 1.47 & 0.69 & 0.26 & 0.58 & $\mathrm{a} * *$ \\
\hline 527 & $3: 57: 57.95$ & $+53: 45: 52.6$ & 12.95 & 3.70 & 2.89 & 2.11 & 0.96 & 0.34 & 0.88 & g1.5 IV \\
\hline 528 & $3: 57: 58.17$ & $+53: 47: 18.8$ & 14.49 & 3.86 & 3.03 & 2.19 & 0.99 & 0.37 & 0.88 & g2.5 III \\
\hline 529 & $3: 57: 58.57$ & $+53: 58: 05.4$ & 14.84 & 3.58: & 2.61 & 1.80 & 0.86 & 0.34 & 0.77 & f4 III \\
\hline 530 & $3: 57: 58.75$ & $+53: 16: 11.9$ & 16.83 & & & 2.61: & 1.04 & 0.55 & 0.98 & $\mathrm{k} 3.2 \mathrm{~V}$ \\
\hline 531 & $3: 57: 59.38$ & $+54: 06: 07.8$ & 13.89 & 3.80 & 2.63 & 1.67 & 0.91 & 0.31 & 0.73 & b9.5 III \\
\hline 532 & $3: 57: 59.54$ & $+53: 23: 45.5$ & 15.18 & 3.52 & 2.76 & 2.04 & 0.93 & 0.39 & 0.86 & f9 IV \\
\hline 533 & $3: 58: 00.62$ & $+53: 09: 15.8$ & 16.35 & & & 2.21 & 0.99 & 0.47 & 0.90 & $\mathrm{k} 0$ : \\
\hline 534 & $3: 58: 01.16$ & $+53: 51: 25.1$ & 14.85 & & & 2.89 & 1.20 & 0.49 & 1.09 & k1 III \\
\hline 535 & $3: 58: 02.00$ & $+53: 32: 45.9$ & 13.07 & 3.80 & 2.85 & 1.79 & 0.80 & 0.28 & 0.70 & $\mathrm{f}$ \\
\hline 536 & $3: 58: 02.21$ & $+54: 05: 59.3$ & 11.86 & 2.84 & 2.27 & 1.60 & 0.73 & 0.27 & 0.69 & $\mathrm{f} 7 \mathrm{~V}$ \\
\hline 537 & $3: 58: 02.31$ & $+53: 42: 33.5$ & 11.19 & 3.45 & 2.64 & 1.80 & 0.84 & 0.31 & 0.76 & f6 III \\
\hline 538 & $3: 58: 02.88$ & $+53: 09: 11.3$ & 16.69 & & & 2.61 & 1.14 & 0.49 & 1.08: & $\mathrm{k} 1 \mathrm{~V}$ \\
\hline 539 & $3: 58: 02.90$ & $+54: 00: 42.4$ & 14.31 & 4.35: & 3.65 & 2.58 & 1.07 & 0.45 & 0.99 & k0 IV \\
\hline 540 & 3:58:02.93 & $+54: 10: 16.5$ & 15.93 & & & 2.89 & 1.29 & 0.52 & 0.95 & k1 IV: \\
\hline 541 & $3: 58: 03.32$ & $+53: 39: 39.8$ & 15.90 & & & 3.17 & 1.32 & 0.57 & 1.22 & $\mathrm{k} * *$ \\
\hline 542 & $3: 58: 03.60$ & $+54: 11: 42.4$ & 11.50 & 3.17 & 2.38 & 1.44 & 0.68 & 0.24 & 0.54 & a5 IV \\
\hline 543 & $3: 58: 04.03$ & $+54: 07: 59.6$ & 15.46 & 3.38 & 2.61 & 1.89 & 0.90 & 0.35 & 0.79 & $\mathrm{f} 4 \mathrm{~V}$ \\
\hline 544 & 3:58:05.34 & $+54: 06: 54.5$ & 13.49 & 3.17 & 2.56 & 1.85 & 0.82 & 0.33 & 0.75 & $\mathrm{~g} 2.5 \mathrm{~V}$ \\
\hline
\end{tabular}


Table 3. Continued

\begin{tabular}{|c|c|c|c|c|c|c|c|c|c|c|}
\hline No. & $\begin{array}{l}\mathrm{RA}(\mathrm{J} 2000) \\
\mathrm{h} \mathrm{m} \text { s }\end{array}$ & $\begin{array}{c}\operatorname{DEC}(\mathrm{J} 2000) \\
\circ, \prime \prime\end{array}$ & $\begin{array}{c}V \\
\text { mag }\end{array}$ & $\begin{array}{l}U-V \\
\operatorname{mag}\end{array}$ & $\begin{array}{l}P-V \\
\text { mag }\end{array}$ & $\begin{array}{l}X-V \\
\text { mag }\end{array}$ & $\begin{array}{l}Y-V \\
\operatorname{mag}\end{array}$ & $\begin{array}{l}Z-V \\
\operatorname{mag}\end{array}$ & $\begin{array}{l}V-S \\
\text { mag }\end{array}$ & $\begin{array}{l}\text { Photom. } \\
\text { sp. type }\end{array}$ \\
\hline 545 & $3: 58: 05.41$ & $+53: 56: 03.0$ & 12.86 & 3.10 & 2.25 & 1.27 & 0.62 & 0.22 & 0.52 & $\mathrm{a} 0.5 \mathrm{IV}$ \\
\hline 546 & $3: 58: 05.69$ & $+53: 51: 50.3$ & 14.29 & 3.34 & 2.41 & 1.42 & 0.67 & 0.25 & 0.53 & a4 IV \\
\hline 547 & 3:58:06.01 & $+53: 54: 56.4$ & 14.78 & & 3.64 & 2.51 & 0.94 & 0.56 & 0.94 & $\mathrm{k} 4 \mathrm{~V}:$ \\
\hline 548 & $3: 58: 06.64$ & $+53: 35: 40.2$ & 14.92 & 3.49: & 2.69 & 1.99 & 0.90 & 0.35 & 0.84 & f8 IV \\
\hline 549 & $3: 58: 06.66$ & $+53: 53: 47.8$ & 13.42 & 3.34 & 2.65 & 1.91 & 0.88 & 0.32 & 0.81 & f8 IV \\
\hline 550 & $3: 58: 06.97$ & $+54: 14: 27.0$ & 16.32 & & & 2.71 & 1.12 & 0.55 & 1.01 & $\mathrm{k} 2.7 \mathrm{~V}$ \\
\hline 551 & $3: 58: 07.06$ & $+53: 55: 28.3$ & 14.45 & 3.24 & 2.45 & 1.73 & 0.82 & 0.31 & 0.73 & f4 IV \\
\hline 552 & $3: 58: 07.07$ & $+53: 10: 53.6$ & 12.98 & 3.16 & 2.25 & 1.28 & 0.63 & 0.23 & 0.50 & $\mathrm{a} 0.5 \mathrm{IV}$ \\
\hline 553 & $3: 58: 07.19$ & $+54: 08: 37.8$ & 14.93 & & & 2.76 & 1.18 & 0.52 & 1.03 & $\mathrm{k} 1 \mathrm{IV}$ \\
\hline 554 & $3: 58: 07.59$ & $+53: 36: 12.9$ & 14.41 & 4.13: & 3.52 & 2.44 & 0.92 & 0.56 & 0.95 & $\mathrm{k} 9 \mathrm{~V}$ : \\
\hline 555 & 3:58:08.09 & $+54: 09: 37.5$ & 14.87 & 3.50: & 2.52 & 1.55 & 0.77 & 0.30 & 0.61 & a1.5 III \\
\hline 556 & $3: 58: 08.57$ & $+53: 58: 01.4$ & 13.80 & 3.96 & 3.16 & 2.28 & 1.03 & 0.40 & 0.95 & g3 IV \\
\hline 557 & $3: 58: 08.61$ & $+53: 28: 30.1$ & 13.86 & 3.47 & 2.50 & 1.51 & 0.73 & 0.25 & 0.58 & a1.5 IV \\
\hline 558 & $3: 58: 08.72$ & $+53: 47: 19.8$ & 16.47 & & & 2.59 & 1.08 & 0.49 & 1.00 & $\mathrm{k} 1.7 \mathrm{~V}$ \\
\hline 559 & $3: 58: 09.43$ & $+53: 24: 03.6$ & 14.61 & 3.64 & 2.74 & 1.96 & 0.91 & 0.34 & 0.82 & f6 III \\
\hline 560 & $3: 58: 10.76$ & $+53: 40: 21.1$ & 14.59 & 3.78: & 2.74 & 1.72 & 0.84 & 0.29 & 0.68 & a4 III \\
\hline 561 & $3: 58: 10.84$ & $+53: 05: 10.8$ & 14.61 & 3.43 & 2.59 & 1.90 & 0.88 & 0.34 & 0.73 & f6 IV \\
\hline 562 & $3: 58: 11.16$ & $+53: 20: 42.8$ & 14.78 & 3.68: & 2.60 & 1.77 & 0.83 & 0.27 & 0.75 & f3 III-IV \\
\hline 563 & $3: 58: 11.87$ & $+53: 28: 50.6$ & 15.97 & & & 2.69 & 1.09 & 0.49 & 1.07 & k0.5 IV \\
\hline 564 & $3: 58: 12.01$ & $+54: 06: 19.3$ & 13.43 & 3.37 & 2.36 & 1.49 & 0.74 & 0.27 & 0.66 & a4 III \\
\hline 565 & $3: 58: 12.07$ & $+53: 43: 30.2$ & 16.00 & & & 2.44 & 1.02 & 0.39 & 0.96 & k0 IV \\
\hline 566 & $3: 58: 12.49$ & $+53: 54: 28.1$ & 13.20 & 3.29 & 2.33 & 1.39 & 0.69 & 0.24 & 0.56 & a0 III \\
\hline 567 & $3: 58: 13.27$ & $+54: 04: 50.6$ & 16.11 & & & 2.91 & 1.21 & 0.63 & 0.93 & $\mathrm{~m} 1 \mathrm{~V}$ \\
\hline 568 & $3: 58: 13.63$ & $+54: 01: 30.5$ & 14.61 & 4.14 & 2.94 & 1.91 & 0.98 & 0.36 & 0.80 & $\mathrm{a} * *$ \\
\hline 569 & $3: 58: 13.88$ & $+53: 04: 16.6$ & 14.68 & 3.86 & 2.69 & 1.82 & 0.83 & 0.31 & 0.72 & $\mathrm{f}$ \\
\hline 570 & $3: 58: 14.99$ & $+53: 06: 05.6$ & 13.54 & 3.04 & 2.17 & 1.23 & 0.59 & 0.21 & 0.44 & a1.5 IV \\
\hline 571 & 3:58:14.99 & $+54: 12: 12.8$ & 15.15 & 3.52 & 2.66 & 1.93 & 0.92 & 0.37 & 0.76 & f4 IV \\
\hline 572 & $3: 58: 15.39$ & $+54: 06: 47.7$ & 12.91 & 3.33 & 2.46 & 1.48 & 0.68 & 0.25 & 0.55 & a8 III \\
\hline 573 & $3: 58: 15.71$ & $+53: 18: 31.4$ & 15.37 & & & 3.42 & 1.43 & 0.62 & 1.34 & k2 III \\
\hline $574^{*}$ & $3: 58: 15.90$ & $+54: 09: 25.6$ & 10.36 & 2.37 & & 1.07 & 0.61 & 0.22 & 0.47 & b2.5 III: \\
\hline 575 & $3: 58: 16.20$ & $+53: 31: 52.0$ & 14.32 & 4.08 & 3.05 & 2.17 & 1.02 & 0.38 & 0.89 & $\mathrm{~g} * *$ \\
\hline 576 & $3: 58: 17.12$ & $+53: 11: 51.8$ & 14.73 & & & 3.69 & 1.61 & 0.66 & 1.41 & $\mathrm{k} 2$ III \\
\hline 577 & $3: 58: 17.77$ & $+53: 42: 01.7$ & 13.39 & 3.96 & 2.81 & 1.74 & 0.85 & 0.31 & 0.72 & a3 III \\
\hline 578 & $3: 58: 20.04$ & $+53: 28: 03.0$ & 13.14 & 3.89 & 2.81 & 1.83 & 0.88 & 0.33 & 0.74 & a7 III \\
\hline 579 & $3: 58: 20.31$ & $+53: 52: 52.8$ & 14.52 & 3.31 & 2.57 & 1.88 & 0.85 & 0.33 & 0.79 & $\mathrm{~g} * *$ \\
\hline 580 & $3: 58: 20.52$ & $+53: 47: 36.6$ & 16.50 & & & 2.58 & 1.02 & 0.47 & 0.99 & k0.7 IV \\
\hline 581 & $3: 58: 21.01$ & $+53: 04: 02.9$ & 14.19 & & & 4.51 & 1.90 & 0.84 & 1.65 & k6 III \\
\hline 582 & $3: 58: 21.42$ & $+54: 14: 17.3$ & 14.14 & & & 4.02 & 1.83 & 0.75 & 1.51 & $\mathrm{k} 1.5 \mathrm{III}$ \\
\hline 583 & $3: 58: 21.47$ & $+53: 56: 28.8$ & 11.02 & 2.92 & 2.37 & 1.62 & 0.73 & 0.28 & 0.67 & f9 IV \\
\hline 584 & $3: 58: 21.60$ & $+53: 53: 57.8$ & 14.26 & 3.51 & 2.61 & 1.74 & 0.82 & 0.31 & 0.67 & $\mathrm{f} * *$ \\
\hline 585 & $3: 58: 22.13$ & $+53: 10: 22.3$ & 14.46 & 3.26 & 2.47 & 1.77 & 0.82 & 0.33 & 0.74 & f3 IV \\
\hline 586 & $3: 58: 22.39$ & $+53: 33: 56.4$ & 14.49 & 3.93: & 3.04 & 2.18 & 1.01 & 0.38 & 0.90 & g2 IV \\
\hline 587 & $3: 58: 22.57$ & $+53: 38: 42.2$ & 14.13 & 3.40 & 2.60 & 1.86 & 0.88 & 0.32 & 0.78 & f5 IV-V \\
\hline 588 & $3: 58: 22.65$ & $+53: 43: 46.4$ & 13.13 & 3.68 & 2.75 & 1.88 & 0.88 & 0.31 & 0.79 & f3 III \\
\hline 589 & $3: 58: 25.05$ & $+53: 34: 16.0$ & 16.83 & & & 2.54 & 1.05 & 0.51 & 1.04 & $\mathrm{k} 2.5 \mathrm{~V}$ \\
\hline 590 & $3: 58: 25.13$ & $+53: 21: 45.2$ & 14.30 & 3.61 & 2.69 & 1.90 & 0.89 & 0.33 & 0.78 & f3 III \\
\hline 591 & $3: 58: 25.18$ & $+53: 37: 11.6$ & 15.88 & & & 2.55 & 1.07 & 0.49 & 1.03 & $\mathrm{k} 1.7 \mathrm{~V}$ \\
\hline 592 & $3: 58: 25.78$ & $+53: 31: 39.7$ & 13.89 & & & 3.82 & 1.63 & 0.66 & 1.48 & k3 III: ** \\
\hline 593 & $3: 58: 25.80$ & $+53: 55: 07.1$ & 14.29 & 3.27 & 2.39 & 1.63 & 0.75 & 0.28 & 0.65 & f4 III \\
\hline 594 & $3: 58: 26.16$ & $+53: 19: 52.7$ & 13.97 & & & 4.31 & 1.79 & 0.80 & 1.63 & k5 III: ** \\
\hline 595 & $3: 58: 26.24$ & $+53: 42: 20.4$ & 14.14 & 3.99 & 2.87 & 1.88 & 0.86 & 0.32 & 0.74 & $\mathrm{f}$ \\
\hline 596 & $3: 58: 26.72$ & $+54: 06: 19.9$ & 13.99 & 4.70 & 3.89 & 2.84 & 1.17 & 0.60 & 1.23 & $\mathrm{k} 3$ \\
\hline 597 & $3: 58: 27.52$ & $+53: 56: 00.4$ & 14.28 & 3.27 & 2.43 & 1.63 & 0.78 & 0.29 & 0.67 & f2 IV \\
\hline 598 & $3: 58: 28.26$ & $+53: 48: 52.0$ & 15.85 & & & 2.56 & 1.07 & 0.51 & 0.96 & $\mathrm{k} 1.7 \mathrm{~V}$ \\
\hline 599 & $3: 58: 28.87$ & $+53: 39: 56.9$ & 16.05 & & & 2.86 & 1.10 & 0.56 & 1.09 & $\mathrm{k} 3 \mathrm{~V}$ \\
\hline 600 & $3: 58: 28.88$ & $+53: 20: 52.3$ & 13.88 & 3.92 & 2.73 & 1.74 & 0.88 & 0.32 & 0.71 & $\mathrm{a} * *$ \\
\hline 601 & $3: 58: 29.39$ & $+53: 56: 57.4$ & 14.91 & 3.59 & 2.67 & 1.94 & 0.94 & 0.36 & 0.81 & f6 IV \\
\hline 602 & $3: 58: 29.49$ & $+54: 07: 34.3$ & 14.79 & 3.65 & 2.62 & 1.68 & 0.81 & 0.30 & 0.64 & a4 III-IV \\
\hline 603 & $3: 58: 29.64$ & $+53: 37: 07.7$ & 14.54 & 3.49 & 2.69 & 1.98 & 0.90 & 0.35 & 0.82 & f8 IV \\
\hline 604 & $3: 58: 29.70$ & $+53: 40: 36.9$ & 14.50 & & & 3.37 & 1.41 & 0.59 & 1.30 & k1.7 III \\
\hline 605 & $3: 58: 31.74$ & $+53: 22: 29.0$ & 16.87 & & & 2.28 & 1.02 & 0.52 & 0.98 & $\mathrm{~g} 7 \mathrm{~V}$ \\
\hline 606 & $3: 58: 31.93$ & $+53: 21: 06.9$ & 11.86 & 4.93 & 4.14 & 2.88 & 1.20 & 0.48 & 1.09 & k0.7 III \\
\hline
\end{tabular}


Table 3. Continued

\begin{tabular}{|c|c|c|c|c|c|c|c|c|c|c|}
\hline No. & $\begin{array}{c}\text { RA (J2000) } \\
\text { h m s }\end{array}$ & $\begin{array}{c}\mathrm{DEC}(\mathrm{J} 2000) \\
\circ, \prime \prime\end{array}$ & $\begin{array}{c}V \\
\text { mag }\end{array}$ & $\begin{array}{l}U-V \\
\text { mag }\end{array}$ & $\begin{array}{l}P-V \\
\text { mag }\end{array}$ & $\begin{array}{l}X-V \\
\text { mag }\end{array}$ & $\begin{array}{l}Y-V \\
\text { mag }\end{array}$ & $\begin{array}{l}Z-V \\
\text { mag }\end{array}$ & $\begin{array}{l}V-S \\
\text { mag }\end{array}$ & $\begin{array}{l}\text { Photom. } \\
\text { sp. type }\end{array}$ \\
\hline 607 & $3: 58: 32.03$ & $+53: 28: 24.5$ & 11.21 & 2.67 & 1.99 & 1.37 & 0.74 & 0.27 & 0.62 & b5 IV: \\
\hline 608 & $3: 58: 32.23$ & $+53: 14: 46.9$ & 14.93 & & $3.47:$ & 2.45 & 1.05 & 0.46 & 0.96 & k0 IV \\
\hline 609 & $3: 58: 34.50$ & $+53: 50: 50.2$ & 11.65 & 2.89 & 2.09 & 1.13 & 0.56 & 0.20 & 0.44 & $\mathrm{a} 0 \mathrm{IV}$ \\
\hline 610 & $3: 58: 35.37$ & $+54: 15: 20.0$ & 16.44 & & & $2.52:$ & 1.21: & $0.61:$ & 1.01 & g1 V \\
\hline 611 & $3: 58: 35.46$ & $+53: 37: 25.8$ & 16.60 & & & 2.76: & 1.15 & 0.53 & 1.12 & $\mathrm{k} 2.2 \mathrm{~V}$ \\
\hline 612 & $3: 58: 35.70$ & $+53: 24: 13.6$ & 16.22 & & & 2.43 & 1.01 & 0.45 & 1.00 & $\mathrm{k} 1.5 \mathrm{~V}$ \\
\hline 613 & $3: 58: 35.73$ & $+53: 06: 26.7$ & 13.64 & 4.87 & 3.91 & 2.81 & 1.21 & 0.46 & 1.07 & g7 III \\
\hline 614 & $3: 58: 35.95$ & $+53: 21: 19.9$ & 16.50 & & & 2.37 & 0.96 & 0.44 & 1.00 & $\mathrm{k} 1.7 \mathrm{~V}$ \\
\hline 615 & $3: 58: 36.06$ & $+54: 07: 14.1$ & 15.35 & & & 2.55 & 1.08 & 0.46 & 1.00 & k0 IV \\
\hline 616 & $3: 58: 37.01$ & $+54: 11: 53.0$ & 14.48 & 3.92 & 3.18 & 2.25 & 1.07 & 0.42 & 0.88 & g0 IV \\
\hline 617 & $3: 58: 37.60$ & $+53: 03: 57.3$ & 12.09 & 2.46 & 1.78 & 1.09 & 0.57 & 0.21 & 0.44 & b7 IV \\
\hline 618 & $3: 58: 38.11$ & $+53: 50: 37.3$ & 14.04 & 4.79 & 3.91 & 2.87 & 1.28 & 0.49 & 1.16 & g5 III \\
\hline 619 & $3: 58: 38.83$ & $+53: 04: 26.5$ & 12.53 & 3.29 & 2.76 & 1.87 & 0.77 & 0.33 & 0.62 & g8 III \\
\hline 620 & $3: 58: 40.06$ & $+53: 34: 46.5$ & 15.06 & 3.87 & 2.86 & 1.92 & 0.91 & 0.35 & 0.78 & a8 IV \\
\hline 621 & $3: 58: 40.18$ & $+53: 58: 30.1$ & 14.88 & 3.90 & 2.79 & 1.44 & $0.87:$ & $0.35:$ & 0.83 & be: \\
\hline 622 & $3: 58: 40.31$ & $+53: 37: 46.5$ & 14.34 & 3.76 & 2.82 & 2.33 & 1.27 & 0.43 & 1.04 & b9 III-IV: \\
\hline 623 & $3: 58: 41.12$ & $+54: 09: 08.6$ & 14.68 & 3.73: & 2.74 & 1.88 & 0.90 & 0.34 & 0.76 & f1 III \\
\hline 624 & $3: 58: 41.36$ & $+53: 03: 19.4$ & 11.77 & 2.84 & 2.19 & 1.54 & 0.71 & 0.26 & & f5 $\mathrm{V}$ \\
\hline 625 & $3: 58: 42.06$ & $+53: 25: 27.4$ & 14.33 & 3.30 & 2.45 & 1.94 & 1.09 & 0.39 & 0.88 & b1 V: \\
\hline 626 & $3: 58: 42.30$ & $+53: 39: 21.9$ & 14.17 & 4.32: & 3.72 & 2.53 & 0.98 & 0.48 & 0.96 & k1 IV: \\
\hline 627 & $3: 58: 43.22$ & $+54: 01: 50.3$ & 14.91 & 3.44: & 2.61 & 1.91 & 0.89 & 0.36 & 0.79 & f5 IV \\
\hline 628 & $3: 58: 44.26$ & $+54: 07: 05.5$ & 14.33 & 3.83 & 3.06 & 2.24 & 1.00 & 0.39 & 0.92 & $\mathrm{~g} * *$ \\
\hline 629 & $3: 58: 44.74$ & $+53: 03: 19.9$ & 14.88 & 3.28 & 2.35 & 1.92 & 1.07 & 0.42 & & b3 \\
\hline 630 & $3: 58: 45.36$ & $+54: 03: 49.1$ & 16.46 & & & 2.79 & 1.27 & 0.58 & 1.10 & k0 V: \\
\hline 631 & $3: 58: 45.40$ & $+53: 37: 52.7$ & 13.76 & 3.36 & 2.48 & 1.70 & 0.81 & 0.29 & 0.73 & f1 IV \\
\hline 632 & $3: 58: 46.74$ & $+53: 27: 54.5$ & 12.40 & 3.51 & 2.58 & 1.57 & 0.73 & 0.26 & 0.62 & a7 III \\
\hline 633 & $3: 58: 48.30$ & $+53: 59: 37.5$ & 14.82 & 3.51 & 2.69 & 1.84 & 0.92 & 0.34 & 0.81 & f1 V \\
\hline 634 & $3: 58: 48.59$ & $+53: 58: 06.5$ & 14.22 & 4.33 & 3.64 & 2.52 & 1.06 & 0.43 & 0.97 & k0 III \\
\hline 635 & $3: 58: 49.48$ & $+53: 27: 09.0$ & 13.25 & 3.77 & 2.73 & 1.73 & 0.80 & 0.30 & 0.67 & $a-f$ \\
\hline 636 & $3: 58: 50.03$ & $+53: 04: 39.2$ & 11.76 & 2.79 & 1.99 & 1.06 & 0.51 & 0.18 & 0.38 & $\mathrm{a} 0.5 \mathrm{~V}$ \\
\hline 637 & $3: 58: 50.10$ & $+53: 37: 59.5$ & 13.57 & & & 3.61 & 1.50 & 0.61 & 1.35 & k3 III \\
\hline 638 & $3: 58: 50.64$ & $+53: 51: 22.0$ & 12.31 & 3.88 & 3.20 & 2.30 & 1.01 & 0.40 & 0.94 & g6 IV \\
\hline 639 & $3: 58: 51.22$ & $+53: 31: 18.3$ & 13.42 & 3.71 & 2.64 & 1.60 & 0.77 & 0.27 & 0.61 & a3 III \\
\hline 640 & $3: 58: 51.23$ & $+54: 06: 29.4$ & 14.53 & 4.29 & 3.06 & 2.02 & 0.96 & 0.35 & 0.80 & f1 IV \\
\hline 641 & $3: 58: 51.34$ & $+53: 23: 15.4$ & 14.95 & & & 3.56 & 1.46 & 0.67 & 0.88 & k2.5 III: \\
\hline 642 & $3: 58: 51.49$ & $+53: 33: 13.0$ & 16.22 & & & 2.82 & 1.19 & 0.48 & 1.12 & k0.5 IV \\
\hline 643 & $3: 58: 52.33$ & $+53: 13: 49.8$ & 10.31 & 5.26 & 4.46 & 3.10 & 1.27 & 0.50 & 1.16 & k1.7 III \\
\hline 644 & $3: 58: 52.68$ & $+53: 48: 28.1$ & 11.86 & 3.31 & 2.40 & 1.38 & 0.66 & 0.24 & 0.55 & a3 IV \\
\hline 645 & $3: 58: 54.34$ & $+53: 25: 56.3$ & 13.41 & 3.73 & 2.81 & 2.23 & 1.22 & 0.44 & 0.98 & b9 IV-V: \\
\hline 646 & $3: 58: 55.15$ & $+54: 02: 57.5$ & 13.23 & $5.23:$ & 4.37 & 3.01 & 1.23 & 0.52 & 1.09 & $\mathrm{k} 1.5$ III \\
\hline 647 & $3: 58: 55.36$ & $+54: 15: 34.0$ & 11.89 & 3.61 & 2.71 & 1.70 & 0.80 & 0.28 & & a8 IV: \\
\hline 648 & $3: 58: 57.26$ & $+53: 40: 39.7$ & 15.04 & & & 3.01 & 1.10 & 0.69 & 1.12 & $\mathrm{k} 7 \mathrm{~V}$ \\
\hline 649 & $3: 58: 57.37$ & $+53: 28: 22.5$ & 14.44 & 4.53 & 3.55 & 2.62 & 1.20 & 0.47 & 1.11 & g1.5 III \\
\hline 650 & $3: 58: 57.97$ & $+54: 13: 20.1$ & 13.73 & 3.85 & 2.99 & 2.20 & 1.11: & 0.41 & & f5 V: \\
\hline 651 & $3: 58: 58.65$ & $+53: 49: 01.2$ & 10.99 & 4.34 & 3.63 & 2.54 & 1.09 & 0.42 & 0.99 & g8 III \\
\hline 652 & $3: 58: 59.33$ & $+53: 54: 10.1$ & 13.72 & 3.91 & 3.21 & 2.30 & 1.00 & 0.40 & 0.92 & g6 III \\
\hline 653 & $3: 59: 01.13$ & $+53: 47: 26.8$ & 14.62 & 3.34 & 2.52 & 1.82 & 0.85 & 0.32 & 0.75 & f6 IV \\
\hline 654 & $3: 59: 03.60$ & $+53: 39: 06.8$ & 16.26 & & & 2.60 & 1.06 & 0.53 & 1.03 & $\mathrm{k} * *$ \\
\hline 655 & $3: 59: 03.87$ & $+53: 26: 03.3$ & 13.38 & 3.41 & 2.47 & 1.48 & 0.68 & 0.24 & 0.55 & a5 III \\
\hline 656 & $3: 59: 04.11$ & $+53: 10: 39.2$ & 12.25 & 3.39 & 2.49 & 1.47 & 0.68 & 0.24 & 0.57 & a6 III \\
\hline 657 & $3: 59: 04.46$ & $+53: 19: 18.4$ & 14.44 & 3.54 & 2.59 & 1.67 & 0.78 & 0.28 & 0.66 & fo III \\
\hline 658 & $3: 59: 04.68$ & $+54: 07: 53.8$ & 13.76 & 4.22 & 3.65 & 2.51 & 0.96 & 0.53 & 0.88 & $\mathrm{k} * *$ \\
\hline 659 & $3: 59: 04.96$ & $+53: 11: 19.4$ & 14.28 & 3.24 & 2.36 & 1.71 & 0.90 & 0.31 & 0.73 & b5 V: \\
\hline 660 & 3:59:05.02 & $+53: 56: 49.3$ & 15.49 & & & 2.91 & 1.24 & 0.50 & 1.16 & k0 IV \\
\hline 661 & $3: 59: 05.66$ & $+53: 16: 33.1$ & 14.03 & 3.59 & 2.58 & 1.74 & 0.81 & 0.30 & 0.70 & f2 III \\
\hline 662 & $3: 59: 05.90$ & $+54: 05: 19.6$ & 14.74 & 4.11 & 2.89 & 2.02 & 0.97 & 0.36 & 0.83 & f8 IV \\
\hline 663 & $3: 59: 05.97$ & $+53: 36: 16.3$ & 13.60 & 3.32 & 2.55 & 1.81 & 0.85 & 0.33 & 0.75 & $\mathrm{f} * *$ \\
\hline 664 & 3:59:06.09 & $+53: 43: 55.6$ & 14.50 & 3.68 & 2.70 & 1.66 & 0.78 & 0.26 & 0.64 & a7 III \\
\hline 665 & $3: 59: 06.95$ & $+53: 26: 25.7$ & 14.36 & & & 3.42 & 1.46 & 0.58 & 1.30 & $\mathrm{k} 1.5$ III \\
\hline 666 & 3:59:07.01 & $+54: 03: 33.8$ & 14.37 & 3.50 & 2.54 & 1.55 & 0.76 & $0.25:$ & 0.61 & a2 IV \\
\hline 667 & $3: 59: 07.28$ & $+53: 54: 22.4$ & 14.53 & 4.18 & 3.00 & 1.93 & 0.96 & 0.35 & 0.81 & a5 III \\
\hline 668 & $3: 59: 07.38$ & $+53: 57: 23.9$ & 16.96 & & & 2.31 & 0.99 & 0.42 & 0.91 & $\mathrm{k} 0 \mathrm{~V}$ \\
\hline
\end{tabular}


Table 3. Continued

\begin{tabular}{|c|c|c|c|c|c|c|c|c|c|c|}
\hline No. & $\begin{array}{l}\mathrm{RA}(\mathrm{J} 2000) \\
\mathrm{h} \mathrm{m} \text { s }\end{array}$ & $\begin{array}{c}\operatorname{DEC}(\mathrm{J} 2000) \\
\circ, \prime \prime\end{array}$ & $\begin{array}{c}V \\
\text { mag }\end{array}$ & $\begin{array}{l}U-V \\
\operatorname{mag}\end{array}$ & $\begin{array}{l}P-V \\
\text { mag }\end{array}$ & $\begin{array}{l}X-V \\
\text { mag }\end{array}$ & $\begin{array}{l}Y-V \\
\operatorname{mag}\end{array}$ & $\begin{array}{l}Z-V \\
\operatorname{mag}\end{array}$ & $\begin{array}{l}V-S \\
\text { mag }\end{array}$ & $\begin{array}{l}\text { Photom. } \\
\text { sp. type }\end{array}$ \\
\hline 669 & $3: 59: 07.51$ & $+53: 38: 35.7$ & 14.61 & 3.46 & 2.76 & 2.00 & 0.89 & 0.35 & 0.83 & g1 IV \\
\hline 670 & $3: 59: 07.53$ & $+54: 09: 40.4$ & 14.42 & 3.85 & 3.22 & 2.26 & 0.94 & 0.41 & 0.81 & g9 IV \\
\hline 671 & $3: 59: 08.15$ & $+54: 00: 13.7$ & 14.64 & 3.40 & 2.50 & 1.58 & 0.73 & 0.28 & 0.58 & a9 III \\
\hline 672 & 3:59:09.09 & $+54: 12: 56.9$ & 16.22 & & & 2.91 & 1.27 & 0.67 & 0.95 & $\mathrm{k} 2 \mathrm{~V}:$ \\
\hline 673 & $3: 59: 09.27$ & $+53: 05: 15.7$ & 15.84 & & & 2.30 & 0.96 & 0.43 & 0.84 & k0 IV \\
\hline 674 & $3: 59: 09.77$ & $+53: 52: 01.7$ & 15.20 & & 3.15 & 2.22 & 0.93 & 0.42 & 0.87 & $\mathrm{k} 0 \mathrm{~V}$ \\
\hline 675 & $3: 59: 09.86$ & $+53: 58: 46.2$ & 14.94 & 3.56 : & 2.77 & 2.01 & 0.90 & 0.36 & 0.84 & g1.5 III \\
\hline 676 & $3: 59: 10.39$ & $+54: 09: 35.9$ & 14.20 & 3.45 & 2.61 & 1.86 & 0.95 & 0.33 & 0.71 & a3 IV: \\
\hline 677 & $3: 59: 11.65$ & $+53: 12: 51.2$ & 14.33 & 3.31 & 2.50 & 1.81 & 0.85 & 0.32 & 0.75 & f4 IV \\
\hline 678 & $3: 59: 12.23$ & $+53: 59: 00.5$ & 11.44 & 3.30 & 2.55 & 2.06 & 1.19 & 0.43 & 0.98 & $\mathrm{~b} * *$ \\
\hline 679 & $3: 59: 12.34$ & $+53: 54: 59.8$ & 16.27 & & & 2.58 & 1.01 & 0.50 & 0.96 & $\mathrm{k} 2.5 \mathrm{~V}$ \\
\hline 680 & $3: 59: 15.47$ & $+53: 57: 47.2$ & 15.11 & 3.36: & 2.40 & 1.44 & 0.74 & 0.27 : & 0.64 & $\mathrm{a} 0 \mathrm{III}$ \\
\hline 681 & $3: 59: 15.95$ & $+53: 56: 27.2$ & 14.40 & 3.27 & 2.42 & 1.65 & 0.78 & 0.30 & 0.70 & $\mathrm{f} * *$ \\
\hline 682 & $3: 59: 16.67$ & $+53: 38: 09.2$ & 12.91 & 2.99 & 2.40 & 1.72 & 0.77 & 0.30 & 0.73 & f9 $\mathrm{V}$ \\
\hline 683 & $3: 59: 16.68$ & $+53: 47: 49.6$ & 12.57 & 3.25 & 2.49 & 1.73 & 0.81 & 0.30 & 0.74 & f4 IV \\
\hline 684 & $3: 59: 16.72$ & $+53: 36: 13.1$ & 14.58 & 3.44 & 2.58 & 1.82 & 0.85 & 0.33 & 0.74 & f3 III \\
\hline 685 & $3: 59: 17.08$ & $+53: 41: 49.6$ & 16.37 & & & 2.58 & 1.07 & 0.51 & 0.99 & $\mathrm{k} 2 \mathrm{~V}$ \\
\hline 686 & $3: 59: 18.57$ & $+53: 38: 10.4$ & 15.10 & & & 2.52 & 1.01: & 0.45 & 1.00 & $\mathrm{k} 1 \mathrm{IV}$ \\
\hline 687 & 3:59:19.09 & $+53: 53: 37.6$ & 15.24 & 3.38 & 2.55 & 1.86 & 0.87 & 0.35 & 0.78 & f3 IV \\
\hline 688 & $3: 59: 19.12$ & $+53: 13: 11.2$ & 16.49 & & & 2.47 & 1.08 & 0.50 & 0.92 & $\mathrm{k} 1 \mathrm{~V}$ \\
\hline 689 & $3: 59: 19.19$ & $+53: 58: 19.7$ & 13.05 & 3.12 & 2.32 & 1.51 & 0.74 & 0.28 & 0.61 & a6 III \\
\hline 690 & $3: 59: 19.34$ & $+54: 09: 10.5$ & 14.19 & 3.38 & 2.56 & 1.82 & 0.88 & 0.35 & 0.72 & f4 IV \\
\hline 691 & $3: 59: 20.22$ & $+53: 25: 31.2$ & 16.63 & & & 2.80: & 1.15 & 0.55 & 1.07 & $\mathrm{k} 2.5 \mathrm{~V}$ \\
\hline 692 & $3: 59: 20.24$ & $+53: 51: 53.4$ & 14.99 & $3.52:$ & 2.67 & 1.96 & 0.91 & 0.34 & 0.82 & f7 IV \\
\hline 693 & $3: 59: 20.49$ & $+53: 17: 18.0$ & 15.26 & 3.71 & 2.75 & 2.05 & 0.96 & 0.38 & 0.87 & f5 III \\
\hline 694 & $3: 59: 20.55$ & $+53: 18: 08.8$ & 15.29 & & & 3.67 & 1.64 & 0.69 & 1.41 & k2 III: ** \\
\hline 695 & $3: 59: 20.57$ & $+53: 38: 50.7$ & 16.58 & & & 2.82: & 1.17 & 0.64 & 1.10 & $\mathrm{k} 3 \mathrm{~V}$ \\
\hline 696 & $3: 59: 23.26$ & $+53: 45: 54.6$ & 12.94 & 3.32 & 2.44 & 1.57 & 0.71 & 0.26 & 0.62 & f1 III \\
\hline 697 & $3: 59: 24.49$ & $+53: 57: 53.5$ & 14.90 & & & 4.15 & 1.77 & 0.75 & 1.53 & k3.7 III \\
\hline 698 & $3: 59: 25.10$ & $+53: 49: 08.0$ & 14.60 & & 3.90: & 2.92 & 1.24 & 0.52 & 1.13 & k0 III \\
\hline 699 & $3: 59: 25.15$ & $+53: 27: 44.0$ & 14.08 & 3.36 & 2.51 & 1.79 & 0.83 & 0.31 & 0.75 & f5 III \\
\hline 700 & $3: 59: 25.50$ & $+53: 11: 44.8$ & 13.78 & 3.56 & 2.73 & 1.96 & 0.90 & 0.34 & 0.79 & f7 III \\
\hline 701 & $3: 59: 25.50$ & $+53: 17: 08.0$ & 16.73 & & & 2.64 & 1.17 & 0.57 & 1.07 & $\mathrm{k} 0 \mathrm{~V}$ \\
\hline 702 & $3: 59: 25.64$ & $+53: 44: 08.2$ & 13.23 & 3.68 & 3.10 & 2.11 & 0.85 & 0.38 & 0.84 & $\mathrm{k} 0.7 \mathrm{~V}$ \\
\hline 703 & $3: 59: 25.78$ & $+53: 53: 17.6$ & 16.06 & & & 2.89 & 1.10 & 0.62 & 1.09 & $\mathrm{k} 4.5 \mathrm{~V}$ \\
\hline 704 & $3: 59: 26.50$ & $+53: 29: 11.0$ & 13.00 & 3.28 & 2.33 & 1.36 & 0.66 & 0.24 & 0.52 & a1 III \\
\hline 705 & $3: 59: 27.00$ & $+53: 30: 30.7$ & 14.02 & 3.32 & 2.58 & 1.88 & 0.86 & 0.32 & 0.78 & f8 IV \\
\hline 706 & $3: 59: 27.70$ & $+53: 50: 26.3$ & 16.86 & & & 2.48: & 1.07 & 0.53 & 0.96 & $\mathrm{k} 1 \mathrm{~V}$ \\
\hline 707 & $3: 59: 27.88$ & $+53: 13: 41.4$ & 13.64 & 3.38 & 2.54 & 1.76 & 0.84 & 0.32 & 0.74 & f2 IV \\
\hline 708 & $3: 59: 28.01$ & $+53: 54: 17.6$ & 14.91 & 3.86 & 2.72 & 1.72 & 0.89 & $0.32:$ & 0.74 & $\mathrm{a} 0.5 \mathrm{III}$ \\
\hline 709 & $3: 59: 28.14$ & $+53: 21: 16.6$ & 14.67 & 4.10 & 2.84 & 2.04 & 1.12 & 0.45 & 0.88 & b5 \\
\hline 710 & $3: 59: 28.23$ & $+53: 30: 03.5$ & 15.23 & 3.40 & 2.59 & 1.90 & 0.87 & 0.33 & 0.79 & f7 III \\
\hline 711 & $3: 59: 28.37$ & $+53: 31: 35.0$ & 15.08 & 3.57 & 2.83 & 2.08 & 0.95 & 0.37 & 0.84 & f9 IV \\
\hline 712 & $3: 59: 28.52$ & $+54: 08: 59.3$ & 14.98 & & & 3.08 & 1.17 & 0.75 & 0.87 & $\mathrm{k} 5.5 \mathrm{~V}:$ \\
\hline 713 & $3: 59: 29.52$ & $+54: 04: 54.8$ & 16.20 & & & 2.98 & 1.34 & 0.60 & 1.16 & k1 IV \\
\hline 714 & $3: 59: 29.56$ & $+53: 56: 46.1$ & 15.27 & & & 2.91 & 1.06 & 0.70 & 1.08 & $\mathrm{k} 5.5 \mathrm{~V}$ \\
\hline 715 & $3: 59: 29.73$ & $+53: 51: 38.7$ & 16.18 & & & 2.85 & 1.27 & 0.62 & 0.99 & $\mathrm{k} 1.5 \mathrm{~V}:$ \\
\hline 716 & $3: 59: 29.80$ & $+53: 22: 49.0$ & 14.78 & 3.75 & 2.65 & 1.71 & 0.84 & 0.31 & 0.69 & a4 III \\
\hline 717 & $3: 59: 30.15$ & $+53: 29: 46.5$ & 13.79 & 3.46 & 2.73 & 1.96 & 0.88 & 0.33 & 0.81 & g0 IV \\
\hline 718 & $3: 59: 30.26$ & $+53: 08: 11.0$ & 16.63 & & & 2.55: & 1.19 & 0.61 & 0.94 & $* *$ \\
\hline 719 & $3: 59: 30.29$ & $+53: 56: 21.8$ & 15.02 & 3.48: & 2.58 & 1.66 & 0.76 & 0.29 & 0.67 & fo III \\
\hline 720 & $3: 59: 30.45$ & $+53: 05: 26.0$ & 13.70 & 3.01 & 2.29 & 1.60 & 0.74 & 0.28 & 0.82 & f4 IV: \\
\hline 721 & $3: 59: 31.30$ & $+53: 40: 47.9$ & 13.62 & 3.65 & 2.58 & 1.59 & 0.78 & 0.29 & 0.66 & a3 III \\
\hline 722 & $3: 59: 32.62$ & $+54: 12: 29.6$ & 14.07 & 3.85 & 2.88 & 2.03 & 1.01 & 0.41 & 0.84 & f1 IV \\
\hline 723 & $3: 59: 33.24$ & $+53: 25: 38.4$ & 14.55 & 3.65 & 2.79 & 2.07 & 0.93 & 0.35 & 0.84 & f9.5 IV \\
\hline 724 & $3: 59: 33.35$ & $+53: 49: 08.4$ & 13.32 & 3.43 & 2.54 & 1.79 & 0.86 & 0.31 & 0.77 & f2 IV \\
\hline 725 & $3: 59: 34.05$ & $+53: 32: 22.5$ & 14.58 & 3.36 & 2.51 & 1.80 & 0.86 & 0.31 & 0.75 & f5 IV-V ** \\
\hline 726 & $3: 59: 34.94$ & $+53: 15: 19.6$ & 14.48 & 4.24 & 2.96 & 1.98 & 1.04 & 0.39 & 0.84 & $\mathrm{~b} * *$ \\
\hline 727 & $3: 59: 36.48$ & $+54: 02: 21.1$ & 14.74 & 3.56 & 2.66 & 1.84 & 0.88 & 0.32 & 0.75 & f2 III \\
\hline 728 & $3: 59: 36.73$ & $+54: 10: 56.4$ & 15.26 & & & 2.04 & 1.24: & 0.46 & & \\
\hline 729 & $3: 59: 37.06$ & $+53: 30: 08.8$ & 13.85 & 3.42 & 2.58 & 1.85 & 0.87 & 0.31 & 0.81 & f7 III \\
\hline 730 & $3: 59: 37.21$ & $+53: 06: 06.3$ & 12.57 & 3.05 & 2.35 & 1.65 & 0.77 & 0.29 & & f4 IV \\
\hline
\end{tabular}


Table 3. Continued

\begin{tabular}{|c|c|c|c|c|c|c|c|c|c|c|}
\hline No. & $\begin{array}{l}\text { RA (J2000) } \\
\text { h m s s }\end{array}$ & $\begin{array}{c}\mathrm{DEC}(\mathrm{J} 2000) \\
\circ, \prime \prime\end{array}$ & $\begin{array}{c}V \\
\operatorname{mag}\end{array}$ & $\begin{array}{l}U-V \\
\text { mag }\end{array}$ & $\begin{array}{l}P-V \\
\text { mag }\end{array}$ & $\begin{array}{l}X-V \\
\text { mag }\end{array}$ & $\begin{array}{l}Y-V \\
\text { mag }\end{array}$ & $\begin{array}{l}Z-V \\
\mathrm{mag}\end{array}$ & $\begin{array}{l}V-S \\
\text { mag }\end{array}$ & $\begin{array}{l}\text { Photom. } \\
\text { sp. type }\end{array}$ \\
\hline 731 & $3: 59: 37.24$ & $+53: 55: 34.5$ & 16.06 & & & 3.33: & 1.52 & 0.65 & 1.35 & $\mathrm{k} 1 \mathrm{IV}$ \\
\hline 732 & $3: 59: 37.81$ & $+53: 50: 33.6$ & 14.65 & 3.32 & 2.46 & 1.80 & 0.85 & 0.31 & 0.74 & f6 IV \\
\hline 733 & $3: 59: 37.82$ & $+54: 08: 35.6$ & 15.12 & & & 2.82 & 1.08 & 0.62 & 1.01 & $\mathrm{k} 4 \mathrm{~V}$ \\
\hline 734 & $3: 59: 37.87$ & $+53: 42: 34.8$ & 14.99 & 3.74 & 2.80 & 2.24 & 1.23 & 0.45 & 0.99 & b8 \\
\hline 735 & $3: 59: 38.51$ & $+53: 30: 41.3$ & 14.40 & 3.35 & 2.50 & 1.79 & 0.85 & 0.31 & 0.75 & f7 IV \\
\hline 736 & $3: 59: 39.05$ & $+53: 13: 30.2$ & 15.58 & & & 2.84 & 1.15 & 0.64 & 1.18 & $\mathrm{k} 3.5 \mathrm{~V}$ \\
\hline 737 & $3: 59: 39.59$ & $+53: 35: 54.8$ & 16.57 & & & 2.87: & 1.25 & 0.67 & 0.96 & $\mathrm{k} 1.5 \mathrm{~V}$ \\
\hline 738 & $3: 59: 39.88$ & $+53: 26: 36.6$ & 13.30 & & 5.18: & 3.66 & 1.44 & 0.64 & 1.29 & $\mathrm{k} 4.2$ III \\
\hline 739 & $3: 59: 43.03$ & $+53: 15: 06.0$ & 13.02 & 3.85 & 2.68 & 1.64 & 0.80 & 0.30 & 0.66 & a-f $* *$ \\
\hline 740 & $3: 59: 45.02$ & $+53: 54: 30.1$ & 15.91 & & & 2.28 & 1.00 & 0.49 & 0.88 & g8 V \\
\hline 741 & $3: 59: 45.72$ & $+53: 22: 21.5$ & 16.83 & & & 2.41 & 1.04 & 0.53 & 1.01 & $\mathrm{k} 1 \mathrm{~V}$ \\
\hline 742 & $3: 59: 45.75$ & $+53: 15: 25.7$ & 14.74 & 3.91 & 2.82 & 1.92 & 0.91 & 0.36 & 0.74 & $\mathrm{f} * *$ \\
\hline 743 & $3: 59: 46.78$ & $+53: 07: 16.5$ & 13.06 & 3.25 & 2.39 & 1.52 & 0.69 & 0.27 & 0.72 & f1 III \\
\hline 744 & $3: 59: 47.11$ & $+53: 49: 16.3$ & 12.54 & 3.40 & 2.49 & 1.65 & 0.78 & 0.28 & 0.71 & f2 III \\
\hline 745 & $3: 59: 47.70$ & $+53: 55: 44.7$ & 13.96 & 3.88 & 3.25 & 2.19 & 1.05 & 0.55 & 1.31 & $\mathrm{f} 7$ \\
\hline 746 & $3: 59: 47.77$ & $+54: 03: 40.6$ & 14.58 & 3.76: & 3.00 & 2.13 & 1.02 & 0.38 & 0.87 & f8 IV \\
\hline 747 & $3: 59: 48.01$ & $+53: 30: 47.8$ & 14.99 & 3.48 & 2.68 & 1.97 & 0.91 & 0.36 & 0.84 & f9 IV \\
\hline 748 & $3: 59: 48.08$ & $+54: 09: 44.9$ & 11.98 & 4.70 & 4.02 & 2.73 & 1.18: & 0.51 & 0.75 & k0.5 III: \\
\hline 749 & $3: 59: 49.16$ & $+53: 04: 25.1$ & 15.59 & & & 2.61 & 0.97 & 0.57 & & k5 V \\
\hline 750 & $3: 59: 50.00$ & $+53: 13: 53.2$ & 14.56 & 3.26 & 2.44 & 1.77 & 0.84 & 0.31 & 0.71 & f6 IV \\
\hline 751 & $3: 59: 50.24$ & $+53: 06: 24.4$ & 14.55 & 3.80 & 3.15 & 2.17 & 0.90 & 0.39 & 0.76 : & g9.5 IV \\
\hline 752 & $3: 59: 50.35$ & $+53: 26: 49.5$ & 11.36 & 4.91 & 4.14 & 2.87 & 1.19 & 0.48 & 1.12 & k1 III \\
\hline 753 & $3: 59: 52.35$ & $+53: 27: 22.7$ & 14.42 & 3.66 & 2.84 & 2.06 & 0.94 & 0.36 & 0.87 & f7 III \\
\hline 754 & $3: 59: 52.47$ & $+53: 52: 29.0$ & 15.43 & 3.43 & 2.66 & 1.96 & 0.87 & 0.31 & 0.83 & f8 IV \\
\hline 755 & $3: 59: 53.18$ & $+54: 11: 24.0$ & 14.41 & 3.79 & 2.72 & 1.67 & 0.94: & 0.35 & 0.84 & b9.5 IV \\
\hline 756 & $3: 59: 53.21$ & $+53: 50: 42.3$ & 14.90 & 3.57: & 2.63 & 1.80 & 0.86 & 0.32 & 0.75 & $\mathrm{f} * *$ \\
\hline 757 & $3: 59: 53.52$ & $+53: 42: 48.9$ & 16.53 & & & 2.53 & 1.13 & 0.51 & 0.98 & $\mathrm{~g} * *$ \\
\hline 758 & $3: 59: 54.27$ & $+53: 11: 52.5$ & 13.52 & 3.27 & 2.45 & 1.67 & 0.79 & 0.30 & 0.84 & f2 III: \\
\hline 759 & $3: 59: 54.50$ & $+53: 42: 25.6$ & 10.68 & 5.16 & 4.36 & 3.12 & 1.28 & 0.55 & 1.18 & k1.5 III \\
\hline 760 & $3: 59: 54.72$ & $+53: 52: 24.5$ & 16.37 & & & 2.40 & 1.10 & 0.51 & 1.01 & $\mathrm{~g} 8 * *$ \\
\hline 761 & $3: 59: 56.18$ & $+53: 53: 13.6$ & 10.61 & 2.71 & 1.93 & 1.04 & 0.54 & 0.19 & 0.46 & b9.5 IV \\
\hline 762 & $3: 59: 57.89$ & $+53: 32: 43.9$ & 13.92 & 4.87 & 4.07 & 2.89 & 1.26 & 0.49 & 1.14 & g9 III \\
\hline 763 & $3: 59: 58.01$ & $+53: 35: 48.3$ & 13.54 & 3.59 & 2.74 & 1.99 & 0.93 & 0.34 & 0.85 & f6 III \\
\hline 764 & $3: 59: 58.14$ & $+53: 39: 29.9$ & 15.07 & & & 3.35 & 1.43 & 0.58 & 1.28 & k1.5 III: ** \\
\hline 765 & 4:00:00.25 & $+53: 45: 47.5$ & 16.07 & & & 2.22 & 0.97 & 0.45 & 0.91 & g8 V \\
\hline 766 & 4:00:00.66 & $+53: 55: 16.5$ & 13.34 & 4.89 & 3.73 & 2.80 & 1.35 & 0.48 & 1.18 & f8 IV \\
\hline 767 & 4:00:00.88 & $+53: 49: 39.5$ & 12.24 & 5.38 & 4.51 & 3.20 & 1.34 & 0.52 & 1.20 & k0 III \\
\hline 768 & 4:00:01.07 & $+53: 41: 01.4$ & 14.31 & 3.47 & 2.62 & 1.87 & 0.89 & 0.34 & 0.80 & f4 IV \\
\hline 769 & 4:00:01.62 & $+53: 48: 14.7$ & 12.41 & 3.02 & 2.17 & 1.24 & 0.60 & 0.21 & 0.50 & $\mathrm{a} * *$ \\
\hline 770 & 4:00:02.12 & $+53: 35: 16.9$ & 13.84 & 4.84 & 4.02 & 2.77 & 1.14 & 0.49 & 1.06 & k1 IV \\
\hline 771 & 4:00:02.16 & $+53: 15: 25.7$ & 13.46 & 2.91 & 2.20 & 1.54 & 0.72 & 0.27 & 0.64 & f3 V \\
\hline 772 & 4:00:02.82 & $+53: 43: 35.3$ & 12.72 & 4.13 & 3.63 & 2.49 & 0.85 & 0.58 & 0.93 & k6 V: \\
\hline 773 & 4:00:03.03 & $+53: 58: 43.2$ & 14.04 & 4.08 & 2.80 & 1.88 & 1.00 & 0.37 & 0.82 & a4 III \\
\hline 774 & 4:00:03.72 & $+54: 01: 06.7$ & 13.20 & 5.05 & 4.20 & 3.00 & 1.30 & 0.51 & 1.15 & g9.5 III \\
\hline 775 & 4:00:03.79 & $+53: 36: 58.9$ & 14.44 & 3.81 & 3.01 & 2.17 & 0.96 & 0.37 & 0.91 & g2 III \\
\hline 776 & 4:00:04.17 & $+53: 09: 30.4$ & 16.62 & & & 2.95: & 1.13 & 0.73 & 1.03 & k6 V \\
\hline 777 & 4:00:04.61 & $+53: 43: 54.7$ & 11.52 & 3.15 & 2.78 & 1.83 & 0.68 & 0.34 & 0.71 & k1.7 V: \\
\hline 778 & 4:00:04.94 & $+54: 09: 39.4$ & 14.81 & 3.60 & 2.80 & 2.06 & 1.13: & 0.41 & 0.88 & b4 IV: \\
\hline 779 & 4:00:04.95 & $+53: 53: 26.2$ & 13.26 & 4.95 & 4.07 & 2.88 & 1.25 & 0.48 & 1.10 & g9.5 III \\
\hline 780 & 4:00:05.45 & $+53: 38: 43.2$ & 13.87 & 3.69 & 2.65 & 1.64 & 0.79 & 0.28 & 0.64 & a3 III \\
\hline 781 & 4:00:06.31 & $+53: 17: 28.9$ & 14.98 & 3.22 & 2.40 & 1.75 & 0.84 & 0.31 & 0.69 & f5 IV \\
\hline 782 & 4:00:08.17 & $+53: 58: 49.9$ & 13.91 & 3.48 & 2.48 & 1.56 & 0.75 & 0.27 & 0.63 & a $* *$ \\
\hline 783 & 4:00:08.31 & $+53: 51: 45.7$ & 14.67 & 3.26 & 2.43 & 1.76 & 0.82 & 0.31 & 0.72 & f6 IV \\
\hline 784 & 4:00:08.73 & $+53: 09: 37.2$ & 14.10 & & & 5.13 & 2.26 & 0.89 & 0.88 & k5 III \\
\hline 785 & 4:00:08.76 & $+53: 31: 10.0$ & 14.31 & 3.44 & 2.53 & 1.76 & 0.83 & 0.31 & 0.76 & f4 III \\
\hline 786 & 4:00:09.47 & $+53: 38: 09.7$ & 15.93 & & & 3.62 & 1.65 & 0.64 & 1.46 & k0.5 III \\
\hline 787 & 4:00:09.53 & $+54: 11: 37.2$ & 14.80 & 3.42 & 2.61 & 2.01 & 1.26: & 0.51 & 0.83 & k1 III: \\
\hline 788 & 4:00:09.78 & $+53: 22: 25.5$ & 12.57 & & & 4.65 & 1.93 & 0.85 & 1.73 & k1 III \\
\hline 789 & 4:00:09.81 & $+53: 44: 00.8$ & 13.52 & 3.50 & 2.78 & 1.96 & 0.90 & 0.34 & 0.82 & $\mathrm{~g} 1.5 \mathrm{III}$ \\
\hline 790 & 4:00:10.87 & $+53: 06: 53.6$ & 14.53 & 3.46 & 2.48 & 1.54 & 0.72 & 0.29 & 0.89 & a7 III: \\
\hline 791 & 4:00:11.13 & $+53: 55: 13.8$ & 16.81 & & & 2.36 & 1.03 & 0.50 & 0.90 & $\mathrm{k}$ \\
\hline 792 & 4:00:11.40 & $+53: 19: 31.8$ & 13.97 & & & 3.41 & 1.42 & 0.62 & 1.22 & k2 III \\
\hline
\end{tabular}


Table 3. Continued

\begin{tabular}{|c|c|c|c|c|c|c|c|c|c|c|}
\hline No. & $\begin{array}{c}\mathrm{RA}(\mathrm{J} 2000) \\
\mathrm{h} \mathrm{m} \text { s }\end{array}$ & $\begin{array}{c}\mathrm{DEC}(\mathrm{J} 2000) \\
\circ, \prime \prime\end{array}$ & $\begin{array}{c}V \\
\operatorname{mag}\end{array}$ & $\begin{array}{l}U-V \\
\mathrm{mag}\end{array}$ & $\begin{array}{l}P-V \\
\mathrm{mag}\end{array}$ & $\begin{array}{l}X-V \\
\text { mag }\end{array}$ & $\begin{array}{l}Y-V \\
\operatorname{mag}\end{array}$ & $\begin{array}{l}Z-V \\
\operatorname{mag}\end{array}$ & $\begin{array}{l}V-S \\
\text { mag }\end{array}$ & $\begin{array}{l}\text { Photom. } \\
\text { sp. type }\end{array}$ \\
\hline 793 & $4: 00: 11.85$ & $+54: 00: 29.9$ & 16.20 & & & $2.59:$ & 1.06 & 0.54 & 0.96 & $\mathrm{k} 3 \mathrm{~V}$ \\
\hline 794 & $4: 00: 12.24$ & $+53: 37: 18.1$ & 13.62 & 3.73 & 2.73 & 1.85 & 0.85 & 0.30 & 0.77 & f2 III \\
\hline 795 & $4: 00: 12.72$ & $+53: 51: 24.3$ & 13.84 & 3.13 & 2.28 & 1.60 & 0.86 & 0.30 & 0.72 & b7 V: \\
\hline 796 & 4:00:13.15 & $+53: 08: 34.1$ & 14.01 & 3.00 & 2.30 & 1.62 & 0.77 & 0.28 & $0.88:$ & f5 IV: \\
\hline 797 & 4:00:13.40 & $+53: 24: 57.5$ & 14.43 & 3.45 & 2.63 & 1.93 & 0.89 & 0.35 & 0.80 & f6 IV \\
\hline 798 & 4:00:13.79 & $+54: 14: 17.2$ & 12.60 & 5.20 & 4.37 & 3.00 & 1.55 & $0.62:$ & $0.75:$ & g5 III: \\
\hline 799 & 4:00:14.06 & $+53: 10: 38.8$ & 14.92 & 3.33 & 2.53 & 1.82 & 0.83 & 0.34 & 0.69 & f7 III \\
\hline 800 & $4: 00: 14.11$ & $+53: 30: 26.5$ & 13.97 & & & 3.79 & 1.63 & 0.68 & 1.42 & $\mathrm{k} 2.5$ III \\
\hline 801 & $4: 00: 14.26$ & $+53: 32: 54.0$ & 13.23 & 3.50 & 2.67 & 1.90 & 0.88 & 0.32 & 0.81 & f4 III \\
\hline 802 & $4: 00: 14.59$ & $+53: 14: 03.8$ & 15.59 & & & 2.34 & 0.96 & 0.40 & 0.82 & k0 IV \\
\hline 803 & 4:00:14.91 & $+53: 46: 27.6$ & 14.25 & 3.25 & 2.43 & 1.74 & 0.83 & 0.31 & 0.75 & f6 IV \\
\hline 804 & 4:00:14.93 & $+53: 33: 13.0$ & 15.18 & 3.71 & 2.82 & 1.97 & 0.95 & $0.36:$ & 0.82 & $\mathrm{f} * *$ \\
\hline 805 & $4: 00: 15.16$ & $+53: 14: 40.0$ & 13.58 & 3.12 & 2.43 & 1.73 & 0.81 & 0.31 & 0.63 & f5 IV \\
\hline 806 & $4: 00: 15.77$ & $+53: 38: 19.9$ & 16.25 & & & 2.84 & 1.17 & 0.53 & 1.10 & $\mathrm{k} 1.7 \mathrm{~V}$ \\
\hline 807 & 4:00:15.79 & $+53: 17: 50.7$ & 11.92 & 2.79 & 2.04 & 1.10 & 0.53 & 0.19 & 0.38 & $\mathrm{a} 0 \mathrm{~V}$ \\
\hline 808 & $4: 00: 16.44$ & $+54: 01: 20.2$ & 10.59 & 2.85 & 2.31 & 1.60 & 0.74 & 0.28 & 0.65 & f6 V \\
\hline 809 & $4: 00: 16.48$ & $+53: 09: 30.6$ & 13.44 & 3.15 & 2.33 & 1.43 & 0.66 & 0.25 & 0.82 : & a8 IV: \\
\hline 810 & $4: 00: 16.88$ & $+53: 38: 06.7$ & 14.13 & & & 4.40 & 1.88 & 0.80 & 1.65 & $\mathrm{k} 4.2$ III \\
\hline 811 & 4:00:17.59 & $+53: 19: 21.1$ & 16.62 & & & 2.31 & 0.99 & 0.44 & 1.00 & $\mathrm{k} 0.5 \mathrm{~V}$ \\
\hline 812 & $4: 00: 17.78$ & $+53: 05: 00.5$ & 13.63 & 3.19 & 2.49 & 1.74 & 0.82 & 0.33 & 0.81 & f5 IV \\
\hline 813 & 4:00:17.89 & $+53: 28: 14.7$ & 14.77 & & & 3.00 & 1.07 & 0.68 & 0.88 & $\mathrm{k} 7 \mathrm{~V}$ \\
\hline 814 & 4:00:18.20 & $+53: 53: 23.9$ & 14.02 & 3.45 & 2.69 & 1.95 & 0.90 & 0.33 & 0.80 & f9 IV \\
\hline 815 & 4:00:18.78 & $+54: 02: 07.3$ & 15.87 & & & 2.76 & 1.16 & 0.63 & 0.91 & k3 V \\
\hline 816 & 4:00:19.06 & $+53: 16: 26.2$ & 12.62 & 2.87 & 2.03 & 1.09 & 0.54 & 0.19 & 0.73 & a1 IV: \\
\hline 817 & $4: 00: 19.37$ & $+54: 06: 19.2$ & 14.92 & 3.67 & 2.93 & 2.06 & 1.12: & $0.40:$ & 0.89: & b5 IV: \\
\hline 818 & 4:00:19.85 & $+53: 08: 57.7$ & 13.54 & 3.63 & 2.84 & 2.03 & 0.93 & 0.38 & 0.80 & f9.5 IV \\
\hline 819 & $4: 00: 19.94$ & $+54: 08: 34.2$ & 12.98 & 4.10 & 3.47 & 2.31 & 1.07: & 0.46 & & g9.5 IV: \\
\hline 820 & $4: 00: 20.76$ & $+53: 04: 51.3$ & 12.45 & 3.48 & 2.61 & 1.78 & 0.85 & 0.30 & 0.73 & f1 IV \\
\hline 821 & $4: 00: 20.95$ & $+53: 58: 58.2$ & 13.26 & 5.19 & 4.35 & 3.01 & 1.27 & 0.52 & 1.11 & k1 III \\
\hline 822 & 4:00:21.17 & $+53: 37: 28.0$ & 12.02 & 3.25 & 2.30 & 1.29 & 0.64 & 0.22 & 0.52 & a2 III \\
\hline 823 & 4:00:21.52 & $+53: 46: 35.7$ & 16.05 & & & 2.42 & 1.01 & 0.41 & 0.97 & k0 IV \\
\hline 824 & 4:00:21.57 & $+53: 08: 39.8$ & 14.43 & 3.94 & 2.64 & 1.71 & 0.94 & 0.35 & 0.86 & b8 \\
\hline 825 & $4: 00: 21.59$ & $+53: 27: 47.9$ & 14.77 & 3.61: & 2.71 & 1.92 & 0.93 & 0.35 & 0.79 & f3 IV \\
\hline 826 & 4:00:22.15 & $+53: 43: 57.7$ & 13.11 & 3.12 & 2.60 & 1.81 & 0.76 & 0.31 & 0.73 & g6 V \\
\hline 827 & 4:00:22.98 & $+53: 45: 26.7$ & 12.31 & 3.14 & 2.47 & 1.73 & 0.80 & 0.29 & 0.73 & f7 IV \\
\hline 828 & $4: 00: 23.82$ & $+54: 05: 05.4$ & 14.48 & 3.54 & 2.63 & 1.92 & $0.96:$ & $0.37:$ & $0.85:$ & a8 III \\
\hline 829 & $4: 00: 23.94$ & $+53: 22: 03.5$ & 13.98 & 3.45 & 2.66 & 1.91 & 0.90 & 0.33 & 0.81 & f5 IV \\
\hline 830 & $4: 00: 24.40$ & $+53: 39: 42.9$ & 14.98 & & & 2.70 & 0.99 & 0.58 & 1.01 & $\mathrm{k} 4.5 \mathrm{~V}$ \\
\hline 831 & $4: 00: 24.53$ & $+53: 14: 32.3$ & 13.40 & 3.07 & 2.27 & 1.46 & 0.69 & 0.26 & 0.55 & fo IV \\
\hline 832 & 4:00:24.59 & $+53: 12: 28.5$ & 11.54 & 2.95 & 2.40 & 1.65 & 0.77 & 0.30 & 1.10 & f7 IV: \\
\hline 833 & $4: 00: 24.91$ & $+53: 20: 30.0$ & 16.48 & & & 2.99 & 1.31 & 0.59 & 1.19 & $\mathrm{k} 1.7 \mathrm{~V}$ \\
\hline 834 & 4:00:25.17 & $+54: 11: 39.9$ & 13.91 & 3.76 & 2.77 & 1.79 & 1.08: & $0.46:$ & 0.83 & \\
\hline 835 & $4: 00: 25.33$ & $+53: 25: 53.0$ & 13.23 & 3.64 & 2.70 & 1.87 & 0.88 & 0.32 & 0.79 & f5 III \\
\hline 836 & 4:00:25.74 & $+53: 07: 41.9$ & 14.33 & 3.76 & 3.03 & 2.09 & 0.92 & 0.37 & 0.82 & g5 III \\
\hline 837 & 4:00:26.84 & $+53: 15: 44.5$ & 13.06 & 2.88 & $2.12:$ & 1.24: & $0.62:$ & $0.24:$ & $0.44:$ & $\mathrm{b} * *$ \\
\hline 838 & $4: 00: 27.65$ & $+53: 38: 02.6$ & 12.51 & 3.37 & 2.59 & 1.82 & 0.85 & 0.32 & 0.77 & f4 III \\
\hline 839 & 4:00:27.90 & $+54: 03: 42.5$ & 11.66 & 3.13 & 2.32 & 1.30 & 0.63 & 0.22 & 0.69 & $\mathrm{a} 2 \mathrm{~V}$ \\
\hline 840 & $4: 00: 28.24$ & $+53: 06: 37.4$ & 14.26 & 3.22 & 2.48 & 1.76 & 0.82 & 0.37 & 0.85 & $\mathrm{f} * *$ \\
\hline 841 & $4: 00: 28.55$ & $+53: 47: 44.2$ & 13.87 & 3.22 & 2.43 & 1.72 & 0.82 & 0.30 & 0.72 & f4 III \\
\hline $842^{*}$ & 4:00:30.03 & $+53: 04: 29.2$ & 9.57 & 2.66 & 1.98 & 1.04 & 0.47 & 0.18 & & a5 IV: \\
\hline 843 & 4:00:30.20 & $+53: 20: 03.5$ & 14.96 & 3.72 & 3.00 & 2.07 & 0.92 & 0.37 & $0.77:$ & g4 IV \\
\hline 844 & 4:00:31.10 & $+53: 44: 07.1$ & 15.42 & & & 2.58 & 1.13 & 0.52 & 0.96 & $\mathrm{k} * *$ \\
\hline 845 & $4: 00: 31.23$ & $+53: 38: 05.5$ & 11.23 & 3.13 & 2.22 & 1.22 & 0.62 & 0.22 & 0.51 & a1.5 III \\
\hline 846 & 4:00:33.31 & $+53: 07: 53.5$ & 16.63 & & & 2.49 & 1.05 & 0.55 & 0.97 & $\mathrm{k} 1 \mathrm{~V}$ \\
\hline 847 & 4:00:33.45 & $+53: 15: 07.2$ & 15.86 & & & 3.42 & 1.59 & 0.62 & 1.02 & k0 III: \\
\hline 848 & 4:00:34.49 & $+53: 43: 26.7$ & 14.45 & 3.49 & 2.76 & 1.96 & 0.86 & 0.35 & 0.79 & g0 IV \\
\hline 849 & 4:00:35.07 & $+54: 07: 40.1$ & 14.26 & 3.79 & 2.76 & 1.64 & 0.95: & $0.40:$ & 0.87 & b pec \\
\hline 850 & 4:00:35.43 & $+53: 07: 10.4$ & 11.74 & 2.72 & 2.10 & 1.41 & 0.67 & 0.24 & $0.63:$ & a8 V: \\
\hline 851 & $4: 00: 35.63$ & $+53: 35: 32.8$ & 16.51 & & & 2.55 & 1.11 & 0.52 & 0.96 & $\mathrm{k} 1 \mathrm{~V}$ \\
\hline 852 & $4: 00: 36.29$ & $+53: 33: 16.9$ & 15.65 & & & 2.68 & 1.15 & 0.50 & 1.05 & $\mathrm{k} 1 \mathrm{IV}$ \\
\hline 853 & 4:00:37.99 & $+53: 07: 44.7$ & 16.70 & & & 2.30 & 1.07 & 0.56 & 0.00 & g3 V \\
\hline 854 & 4:00:38.36 & $+53: 59: 40.6$ & 13.87 & 3.65 & 2.75 & 1.75 & 0.82 & 0.31 & 0.69 & f4 IV \\
\hline
\end{tabular}


Table 3. Continued

\begin{tabular}{|c|c|c|c|c|c|c|c|c|c|c|}
\hline No. & $\begin{array}{c}\text { RA (J2000) } \\
\text { h m s s }\end{array}$ & $\begin{array}{c}\operatorname{DEC}(\mathrm{J} 2000) \\
\circ, \prime \prime\end{array}$ & $\begin{array}{c}V \\
\operatorname{mag}\end{array}$ & $\begin{array}{l}U-V \\
\text { mag }\end{array}$ & $\begin{array}{l}P-V \\
\text { mag }\end{array}$ & $\begin{array}{l}X-V \\
\text { mag }\end{array}$ & $\begin{array}{l}Y-V \\
\text { mag }\end{array}$ & $\begin{array}{l}Z-V \\
\text { mag }\end{array}$ & $\begin{array}{l}V-S \\
\operatorname{mag}\end{array}$ & $\begin{array}{l}\text { Photom. } \\
\text { sp. type }\end{array}$ \\
\hline 855 & $4: 00: 38.83$ & $+53: 08: 15.9$ & 14.82 & 3.33 & 2.56 & 1.85 & 0.86 & 0.35 & 1.00 & f5 IV: \\
\hline 856 & 4:00:39.07 & $+53: 22: 34.5$ & 13.72 & 4.36 & 3.37 & 2.38 & 1.11 & 0.43 & 0.95 & g1.5 III \\
\hline 857 & $4: 00: 39.76$ & $+54: 07: 17.7$ & 16.03 & & & 2.74 & 1.30 & $0.65:$ & 1.00 & g6 V: \\
\hline 858 & 4:00:40.32 & $+53: 39: 12.9$ & 13.03 & & 4.82 & 3.46 & 1.43 & 0.62 & 1.32 & k2.2 III \\
\hline 859 & 4:00:41.17 & $+53: 54: 59.9$ & 15.08 & & & 2.89 & 1.10 & 0.69 & 0.86 & $\mathrm{~m} 1 \mathrm{~V}$ \\
\hline 860 & 4:00:41.17 & $+53: 17: 35.6$ & 15.54 & & & 2.34 & 1.02 & 0.46 & 0.91 & k0.7 V \\
\hline 861 & 4:00:41.88 & $+53: 11: 51.9$ & 14.07 & 3.22 & 2.49 & 1.76 & 0.81 & 0.32 & 0.00 & f6 IV \\
\hline 862 & 4:00:43.62 & $+53: 25: 43.0$ & 14.26 & 3.72 & 2.59 & 1.71 & 0.93 & 0.33 & 0.71 & b9 III \\
\hline 863 & $4: 00: 43.83$ & $+53: 17: 21.1$ & 12.54 & 3.63 & 2.98 & 2.01 & 0.86 & 0.37 & 0.74 & g5.5 III \\
\hline 864 & $4: 00: 44.35$ & $+53: 40: 02.3$ & 10.78 & 3.18 & 2.45 & 1.67 & 0.78 & 0.28 & 0.74 & f3 IV \\
\hline 865 & 4:00:45.05 & $+53: 18: 52.9$ & 15.78 & & & 3.39 & 1.47 & 0.63 & $0.98:$ & k1.5 III: \\
\hline 866 & 4:00:46.10 & $+53: 46: 50.0$ & 15.08 & 3.44 & 2.55 & 1.68 & 0.81 & 0.30 & 0.70 & f1 IV \\
\hline 867 & 4:00:46.81 & $+53: 32: 22.6$ & 14.91 & 3.66 & 2.84 & 2.06 & 0.95 & 0.37 & 0.85 & f7 III \\
\hline 868 & 4:00:46.91 & $+53: 44: 13.6$ & 13.25 & 3.28 & 2.36 & 1.38 & 0.68 & 0.26 & 0.48 & a1.5 IV \\
\hline 869 & 4:00:47.08 & $+53: 07: 46.1$ & 12.81 & 4.86 & 3.98 & 2.78 & 1.17 & 0.48 & 0.99 & k0 III \\
\hline 870 & 4:00:47.50 & $+53: 45: 55.5$ & 14.62 & 3.06 & 2.29 & 1.61 & 0.76 & 0.28 & 0.66 & f4 III \\
\hline 871 & 4:00:47.94 & $+53: 36: 07.8$ & 16.68 & & & 2.62 & 1.11 & 0.54 & 0.99 & $\mathrm{k} 2 \mathrm{~V}$ \\
\hline 872 & 4:00:48.40 & $+53: 33: 19.4$ & 13.82 & & 4.10 & 2.94 & 1.26 & 0.50 & 1.09 & k0 III \\
\hline 873 & 4:00:48.59 & $+53: 41: 26.2$ & 12.02 & 3.41 & 2.46 & 1.44 & 0.69 & 0.25 & 0.57 & a4 III \\
\hline 874 & 4:00:48.84 & $+53: 27: 37.5$ & 15.45 & & & 3.22 & 1.12 & 0.78 & & $\mathrm{k} 7 \mathrm{~V}$ \\
\hline 875 & 4:00:49.63 & $+54: 03: 25.6$ & 14.80 & 3.68: & 2.86 & 2.07 & 1.05 & $0.47:$ & 0.98 & $a-f$ \\
\hline $876^{*}$ & 4:00:49.68 & $+53: 22: 37.3$ & 9.93 & 1.88 & 1.45 & 1.02 & 0.49 & 0.20 & 0.45 & a1.5 IV: \\
\hline 877 & 4:00:49.88 & $+53: 04: 55.9$ & 15.51 & & & 3.41 & 1.49 & 0.67 & 0.97 & k1.2 III: \\
\hline 878 & 4:00:51.07 & $+54: 04: 14.3$ & 15.94 & & & 3.37 & 1.56 & 0.66 & & $\mathrm{k} 1.5 \mathrm{~V}$ \\
\hline 879 & 4:00:51.66 & $+53: 54: 28.3$ & 15.69 & & & 3.49 & 1.60 & 0.64 & 0.99 & k0.5 III: \\
\hline 880 & $4: 00: 51.77$ & $+53: 48: 53.3$ & 13.68 & 3.12 & 2.30 & 1.35 & 0.63 & 0.23 & 0.52 & a6 IV \\
\hline $881^{*}$ & 4:00:52.03 & $+53: 22: 45.8$ & 10.22 & 1.96 & 1.50 & 0.98 & 0.50 & 0.21 & 0.32 & b5 IV: \\
\hline 882 & $4: 00: 52.12$ & $+53: 12: 26.5$ & 14.51 & 3.69 & 2.91 & 2.09 & 0.93 & 0.41 & 0.79 & g2 IV \\
\hline 883 & $4: 00: 52.52$ & $+53: 18: 22.0$ & 11.38 & 2.61 & 2.08 & 1.41 & 0.64 & 0.23 & 0.57 & f6 $V:$ \\
\hline 884 & 4:00:53.66 & $+54: 07: 03.7$ & 16.67 & & & 2.49: & 1.33: & $0.75:$ & 1.04 & $\mathrm{a}$ \\
\hline 885 & $4: 00: 55.27$ & $+53: 45: 29.1$ & 12.82 & 3.21 & 2.36 & 1.51 & 0.71 & 0.28 & 0.62 & fO III \\
\hline 886 & 4:00:56.48 & $+53: 10: 19.4$ & 16.75 & & & 2.23 & 0.94 & 0.44 & 1.05 & $\mathrm{k} 1 \mathrm{~V}$ \\
\hline 887 & 4:00:57.79 & $+53: 29: 26.1$ & 14.51 & 3.75 & 2.79 & 2.00 & 0.94 & $0.35:$ & 0.90 & f6 III \\
\hline 888 & $4: 00: 57.96$ & $+53: 29: 09.8$ & 14.67 & 3.73 & 2.80 & 2.04 & 0.92 & $0.37:$ & 0.76 & f9 IV \\
\hline 889 & $4: 00: 58.24$ & $+53: 31: 26.9$ & 15.33 & & & 2.43 & 1.02 & 0.47 & 0.93 & $\mathrm{k} 1.5 \mathrm{~V}$ \\
\hline 890 & 4:00:58.27 & $+53: 48: 33.0$ & 15.44 & 3.42 & 2.65 & 1.93 & 0.88 & 0.34 & 0.75 & f7 III \\
\hline 891 & $4: 00: 59.26$ & $+53: 47: 41.3$ & 15.34 & 3.36 & 2.58 & 1.90 & 0.88 & 0.35 & 0.74 & f5 IV \\
\hline 892 & 4:00:59.52 & $+53: 37: 38.9$ & 13.85 & 3.32 & 2.64 & 1.87 & 0.84 & 0.32 & 0.77 & g1.5 III \\
\hline 893 & 4:01:00.05 & $+53: 21: 37.4$ & 13.63 & 3.14 & 2.47 & 1.75 & 0.81 & 0.32 & $0.62:$ & f7 IV \\
\hline 894 & 4:01:00.82 & $+53: 09: 00.8$ & 12.49 & 3.00 & 2.16 & 1.17 & 0.58 & 0.20 & 0.42 & a1 IV \\
\hline 895 & 4:01:01.37 & $+53: 50: 46.5$ & 12.96 & 3.56 & 2.77 & 1.99 & 0.95 & 0.37 & 0.75 & f6 IV \\
\hline 896 & 4:01:02.16 & $+53: 07: 52.0$ & 13.40 & 3.35 & 2.74 & 1.87: & $0.87:$ & 0.33 & 0.76 & g0 IV \\
\hline 897 & 4:01:02.67 & $+53: 46: 24.4$ & 14.61 & 3.28 & 2.53 & 1.83 & 0.85 & 0.34 & 0.76 & f5 IV \\
\hline 898 & 4:01:02.77 & $+53: 38: 55.1$ & 13.54 & 4.91 & 4.13 & 2.86 & 1.23 & 0.47 & 1.04 & k0 III \\
\hline 899 & 4:01:02.84 & $+53: 08: 29.4$ & 13.12 & 3.97 & 3.26 & 2.27 & 1.00 & 0.37 & 0.88 & g5 III \\
\hline 900 & 4:01:03.11 & $+53: 40: 20.0$ & 13.69 & 3.26 & 2.38 & 1.38 & 0.65 & 0.25 & 0.48 & $\mathrm{a} * *$ \\
\hline 901 & 4:01:03.40 & $+53: 32: 44.1$ & 13.27 & 3.13 & 2.39 & 1.68 & 0.79 & 0.29 & 0.64 & f4 IV \\
\hline 902 & 4:01:03.77 & $+53: 40: 57.7$ & 16.34 & & & 2.32 & 1.00 & 0.45 & & $\mathrm{k} 1 \mathrm{~V}$ \\
\hline 903 & 4:01:03.77 & $+53: 58: 25.9$ & 16.64 & & & 2.32 & 1.03 & 0.49 & & $\mathrm{k} 0 \mathrm{~V}$ \\
\hline 904 & 4:01:03.85 & $+54: 00: 04.7$ & 14.26 & 3.55 & 2.81 & 1.99 & 1.05: & $0.43:$ & 0.90: & $\mathrm{a}$ \\
\hline 905 & 4:01:04.05 & $+53: 52: 09.1$ & 12.58 & 2.99 & 2.42 & 1.72 & 0.76 & 0.30 & 0.61 & g1 V \\
\hline 906 & 4:01:05.06 & $+53: 49: 02.5$ & 14.66 & & & 2.84 & 1.19 & 0.51 & 1.06 & k1 IV \\
\hline 907 & 4:01:05.63 & $+53: 43: 51.8$ & 14.77 & 3.38 & 2.62 & 1.86 & 0.85 & 0.32 & 0.74 & f8 IV \\
\hline 908 & 4:01:05.86 & $+53: 29: 46.5$ & 12.01 & 3.22 & 2.49 & 1.75 & 0.82 & 0.29 & 0.67 & f5 IV \\
\hline 909 & 4:01:06.94 & $+53: 07: 44.3$ & 13.26 & 3.10 & 2.44 & 1.66 & 0.86 & 0.42 & 0.85 & $\mathrm{a} 0 \mathrm{~V}:$ \\
\hline 910 & 4:01:07.17 & $+53: 34: 30.1$ & 12.61 & 3.17 & 2.36 & 1.55 & 0.74 & 0.26 & 0.61 & f1 III \\
\hline 911 & 4:01:07.65 & $+54: 00: 13.5$ & 16.40 & & & 2.46 & 1.17 & $0.57:$ & 1.03 & g2 V: \\
\hline 912 & 4:01:08.31 & $+53: 41: 04.3$ & 14.98 & 3.86 & 2.69 & 1.69 & 0.77 & 0.30 & 0.60 & a4 III \\
\hline 913 & $4: 01: 08.48$ & $+53: 12: 28.6$ & 11.54 & 2.55 & 2.00 & 1.32 & 0.62 & $0.27:$ & 0.93 & f4 IV: \\
\hline 914 & 4:01:09.00 & $+53: 09: 43.3$ & 13.27 & 4.25 & 3.59 & 2.43 & 1.07 & 0.52 & 0.89 & k0 V: \\
\hline 915 & 4:01:10.41 & $+53: 16: 49.5$ & 14.81 & & & 2.92 & 0.99 & 0.74 & 0.84 & $\mathrm{k} 7 \mathrm{~V}$ : \\
\hline 916 & $4: 01: 10.57$ & $+53: 27: 08.7$ & 14.53 & 3.45 & 2.57 & 1.81 & 0.87 & 0.33 & 0.97 & f3 IV: \\
\hline
\end{tabular}


Table 3. Continued

\begin{tabular}{|c|c|c|c|c|c|c|c|c|c|c|}
\hline No. & $\begin{array}{c}\mathrm{RA}(\mathrm{J} 2000) \\
\mathrm{h} \mathrm{m} \mathrm{s}\end{array}$ & $\begin{array}{c}\operatorname{DEC}(\mathrm{J} 2000) \\
\circ, \prime \prime\end{array}$ & $\begin{array}{c}V \\
\text { mag }\end{array}$ & $\begin{array}{l}U-V \\
\operatorname{mag}\end{array}$ & $\begin{array}{l}P-V \\
\text { mag }\end{array}$ & $\begin{array}{l}X-V \\
\text { mag }\end{array}$ & $\begin{array}{l}Y-V \\
\text { mag }\end{array}$ & $\begin{array}{l}Z-V \\
\text { mag }\end{array}$ & $\begin{array}{l}V-S \\
\text { mag }\end{array}$ & $\begin{array}{l}\text { Photom. } \\
\text { sp. type }\end{array}$ \\
\hline 917 & 4:01:11.89 & $+53: 36: 26.3$ & 12.30 & & 5.20 & 3.61 & 1.42 & 0.63 & 1.28 & k3.7 III \\
\hline 918 & $4: 01: 12.76$ & $+54: 05: 57.8$ & 13.50 & 3.50 & 2.70 & 1.83 & 1.12: & 0.48: & 0.81 & $\mathrm{~b}$ \\
\hline 919 & $4: 01: 13.92$ & $+53: 47: 00.2$ & 14.77 & 3.23 & 2.43 & 1.79 & 0.97 & 0.38 & 0.70 & b6 V: \\
\hline 920 & $4: 01: 14.06$ & $+53: 49: 31.0$ & 15.21 & & & 2.54 & 1.08 & 0.45 & 0.98 & k0 III \\
\hline 921 & $4: 01: 14.60$ & $+53: 59: 37.5$ & 13.56 & 3.37 & 2.55 & 1.74 & $0.96:$ & $0.40:$ & $0.78:$ & $\mathrm{b} * *$ \\
\hline 922 & 4:01:15.97 & $+53: 50: 54.5$ & 13.84 & 3.33 & 2.63 & 1.85 & 0.86 & 0.33 & 0.86 & f7 IV: \\
\hline
\end{tabular}

\section{Notes:}

3. HD 24094 (B8), BD+53 708, CY Cam, B2 (Rydström 1978), B1 III (Bouigue 1959); 72. HD 24203 (G0), BD+52 722, dG0 (Rydström 1978); WDS J03537+5316AB, sep. $0.2^{\prime \prime}$ (Hartkopf et al. 2008); 94. ALS 7793, B1 V (Hiltner 1956); 111. BD+53 710, ALS 7795, B5 (Rydström 1978); 235. HD 24386 (F8), BD+53 712, F8 V (Boulon et al. 1958); 279. HD 232856 (G5), BD+53 713, G0 III; 391. WDS J03568+5335A, sep. 9.7", 11.0/13.8 mag, M1.5; 404. NSVS 1985024, EB: (Wozniak et al. 2004; Hoffman et al. 2009); 490. BD+53 715; 574. BD+53 716, B5 (Rydström 1978); 842. HD 232867 (A2), BD+52 732; 876. BD+52 736A, WDS J04008+5323A, A0 (AGK3), B5 (Rydström 1978); 881. BD+52 736B, WDS J04008+5323B. 